\title{
Roles of the Nedd4 Family E3 Ligases in Glial Function and Nerve Cell Development
}

\section{Doctoral Thesis}

\author{
in partial fulfilment of the requirements for the degree \\ "Doctor rerum naturalium" \\ in the Neuroscience Program \\ at the Georg-August-Universität Göttingen, \\ Faculty of Biology
}

submitted by

\section{Bekir Altas}

born in

Kirsehir, Turkey

Göttingen 2016

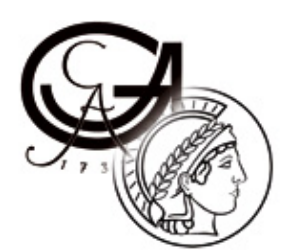


Supervisor

Dr. Hiroshi Kawabe

Department of Molecular Neurobiology

Max Planck Institute for Experimental Medicine, Göttingen

Members of the thesis committee

Prof. Nils Brose, Reviewer

Department of Molecular Neurobiology

Max Planck Institute for Experimental Medicine, Göttingen

Dr. Judith Stegmüller, Reviewer

Department of Cellular and Molecular Neurobiology

Max Planck Institute for Experimental Medicine, Göttingen

Prof. Dirk Görlich

Department of Cellular Logistics

Max Planck Institute for Biophysical Chemistry

Date of the oral examination: $11^{\text {th }}$ May, 2016 


\section{DECLERATION}

I hereby declare that this thesis entitled "Roles of the Nedd4 Family E3 Ligases in Glial Function and Nerve Cell Development" has been written independently, with no other aids than those quoted.

Bekir Altas

March, 2016

Göttingen, Germany 


\section{TABLE OF CONTENTS}

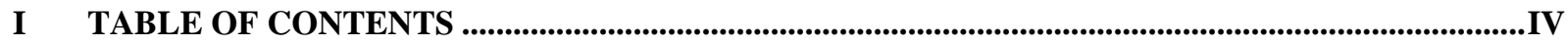

II SUMMARY ................................................................................................................................................................. VII

1 INTRODUCTION ............................................................................................................................ 1

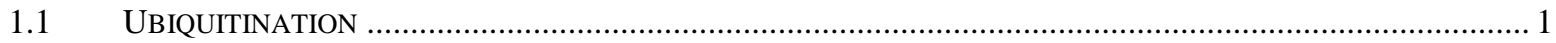

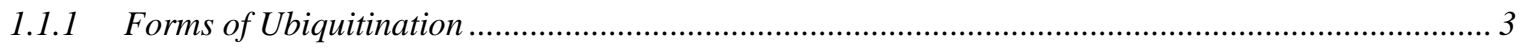

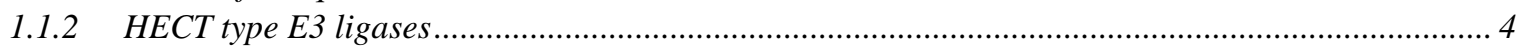

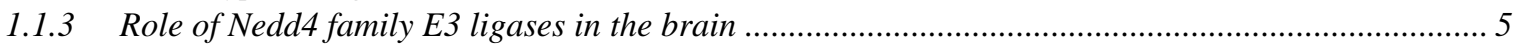

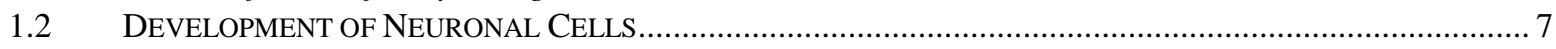





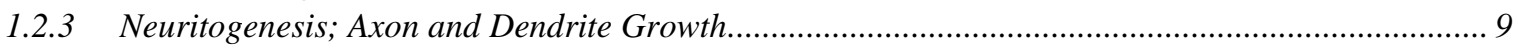

1.3 CONTRIBUTION OF ASTROCYTES TO NEURONAL NETWORKS …...................................................... 11

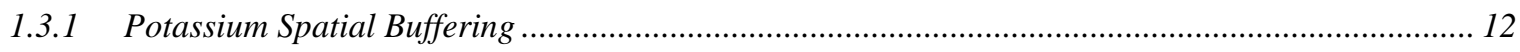

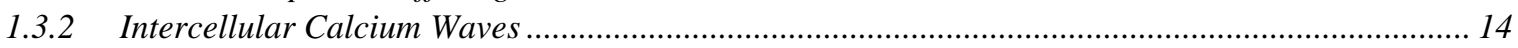

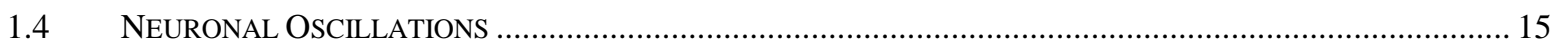

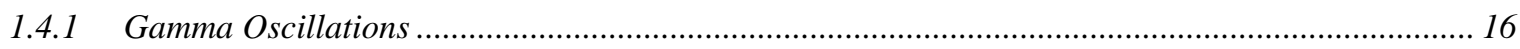

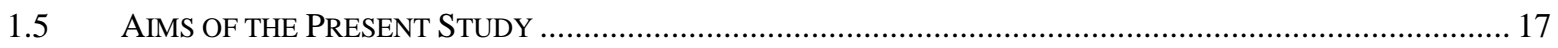

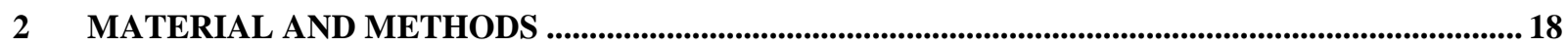



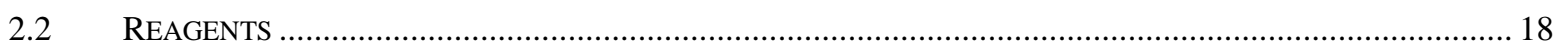

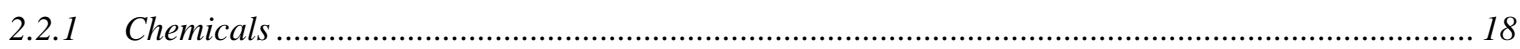

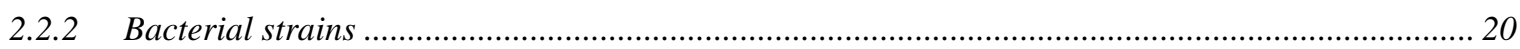



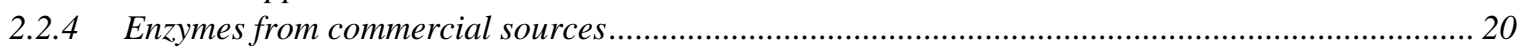

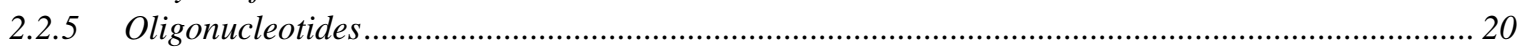

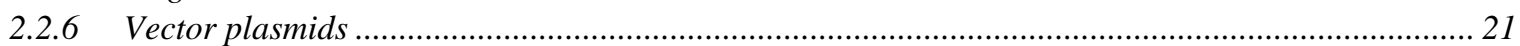

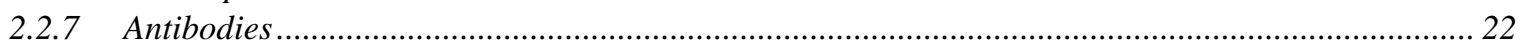

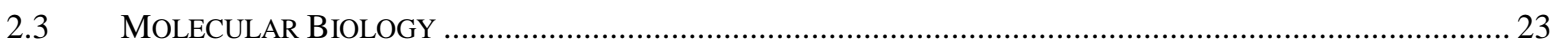

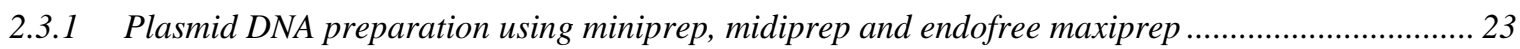

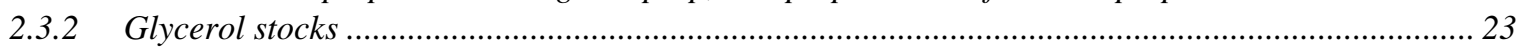

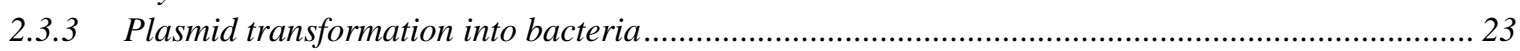

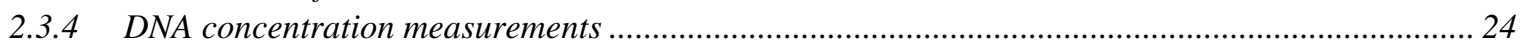

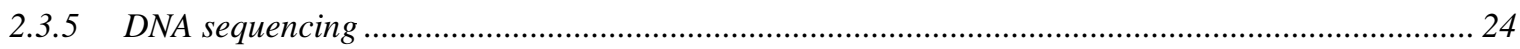

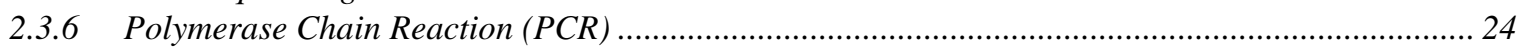

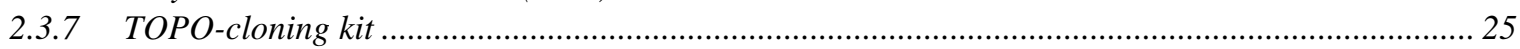

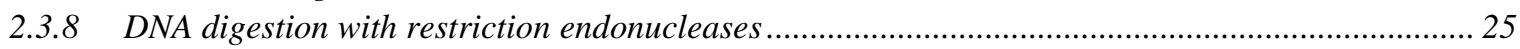

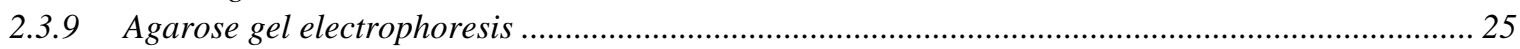

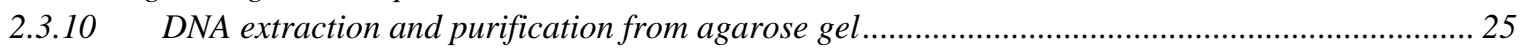

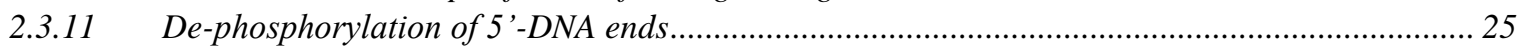



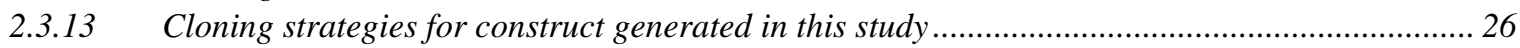

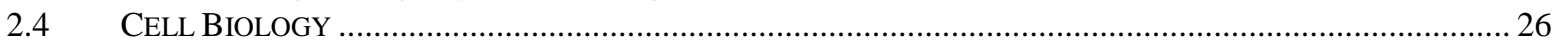

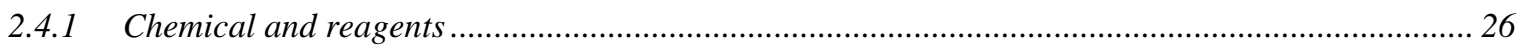



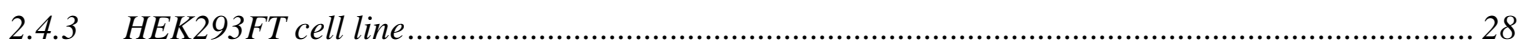

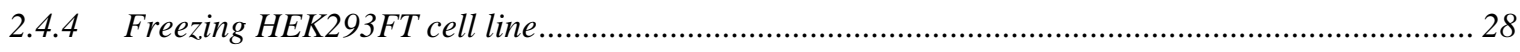

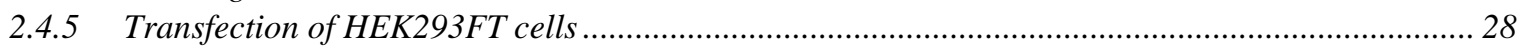




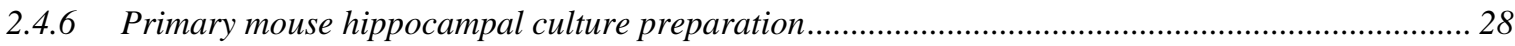

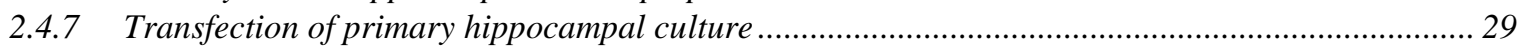

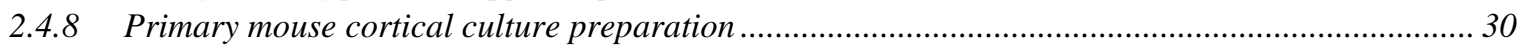

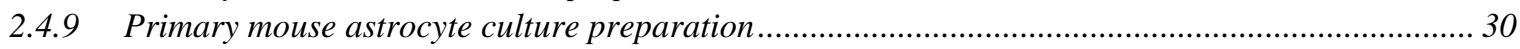

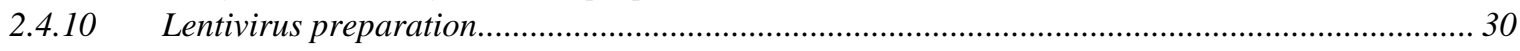

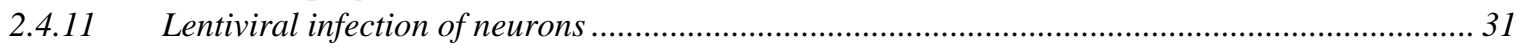

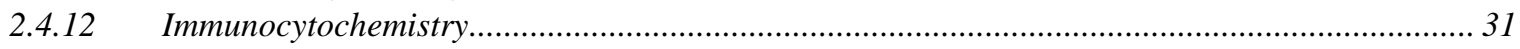

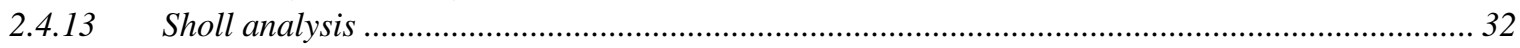

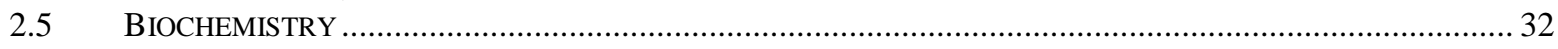

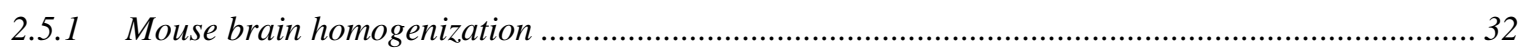

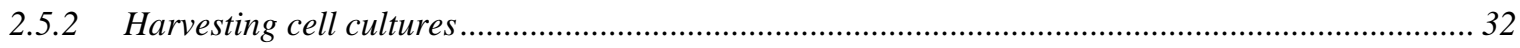

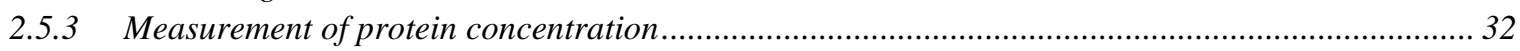

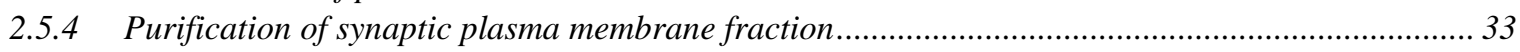





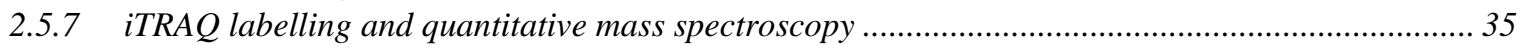

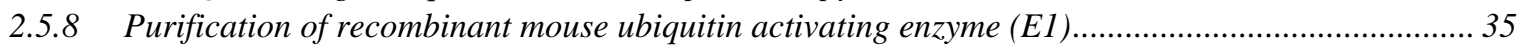

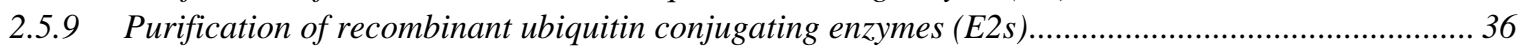

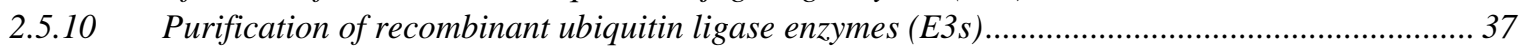



2.5.12 In vitro Ubiquitination Assay for Substrates Expressed in HEK293FT cells............................... 38

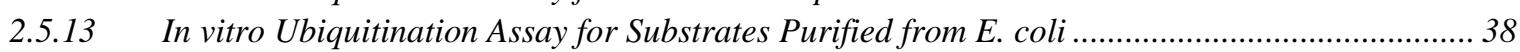

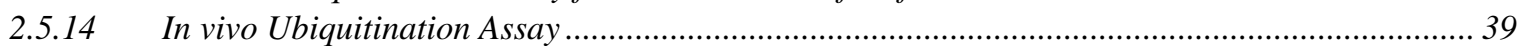

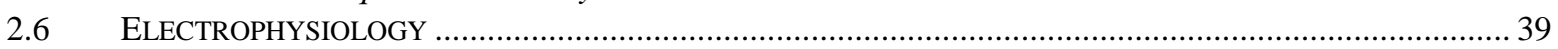

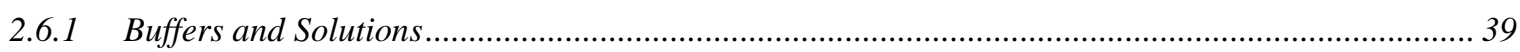

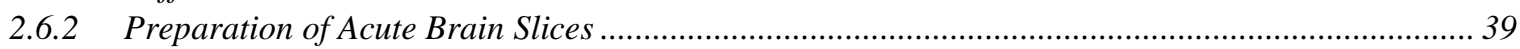

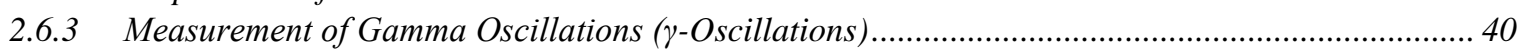

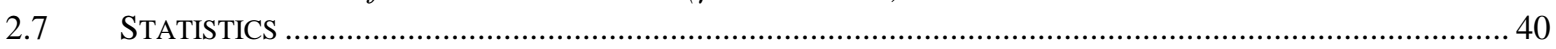

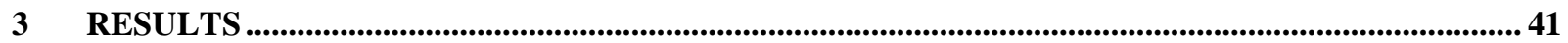

3.1 FUNCTIONS OF NEDD4 SUBFAMILY E3 LIGASES IN THE DEVELOPING AND ADULT BRAIN ....................... 41

3.1.1 Nedd4 family E3 ligases in astrocytes play important roles in neuronal network function............... 41

3.1.1.1 Proteome screening identified Kir4.1, Connexin43, and Prr7 as substrates of Nedd4-1 and Nedd4-2 in

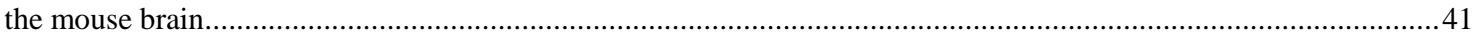

3.1.1.2 The cytoplasmic C-terminal region of Kir4.1 is ubiquitinated by Nedd4-1 and Nedd4-2 E3 ligases........43

3.1.1.3 Full-length Kir4-1 is ubiquitinated in vivo by Nedd4-1 and Nedd4-2 E3 ligases ......................................45

3.1.1.4 Nedd4-2 is a physiological E3 ligase for Kir4.1 ………………….................................................... 47

3.1.1.5 Connexin-43 is ubiquitinated by Nedd4-1 and Nedd4-2 E3 Ligases in vivo ............................................48

3.1.1.6 Connexin-43 levels are regulated by Nedd4-1 and Nedd4-2 mediated ubiquitination ..............................52

3.1.1.7 Hippocampal circuitry in Nedd4-1/2 bDKO mice is imbalanced ..........................................................54

3.1.1.8 Altered gamma oscillations in Nedd4-1/2 bDKO are caused by loss of glial Nedd4-1 and Nedd4-2 ......57

3.1.1.9 Nedd4-2 bKO mice show reduced gamma oscillations in the CA3 pyramidal layer of the hippocampus 59

3.1.2 Nedd4 subfamily E3 ligases might play a role in spine maturation................................................ 61

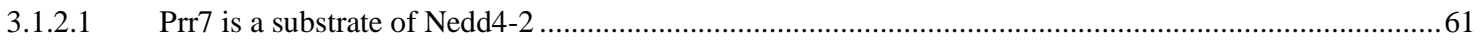

3.1.2.2 Prr7 is conjugated with K63 polyubiquitin chains by Nedd4-1 and Nedd4-2 E3 ligases in vivo..............61

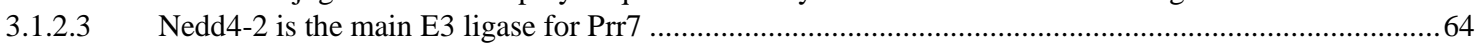

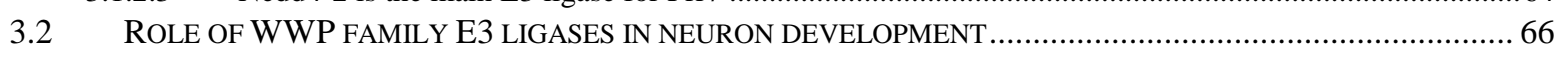

3.2.1 WWP family E3 ligases play an important role in dendrite branching ......................................... 66

3.2.1.1 VCP and CAPZA1 were identified as substrates of WWP1 and WWP2 with proteome screening for less

ubiquitinated proteins in the WWP1;WWP2 double KO mouse brain .............................................................66

3.2.1.2 WWP1/2 double knockout neurons show enhanced neurite growth.................................................69

3.2.1.3 CAPZA1 overexpression in wild-type hippocampal neurons phenocopies the dendrite branching effect of WWP1/2 nDKO. 


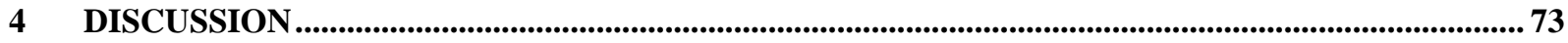

4.1 FUNCTION OF THE NEDD4 SUBFAMILY E3 LIGASES IN MOUSE BRAIN ...................................................73

4.1.1 Novel candidate proteins as substrates of Nedd4-1 or Nedd4-2 are identified in a physiological substrate screening in mouse brain ..................................................................................................... 73

4.1.2 Astrocytic Nedd4-1 and Nedd4-2 are important for the regulation of Kir4.1 and Connexin-43 ..... 74

4.1.3 Kir4.1 is a substrate of Nedd4 subfamily E3 ligases in astrocytes ............................................... 75

4.1.4 Nedd4-2 is the dominant E3 ligase regulating Connexin-43 in astrocytes.................................... 77

4.1.5 Nedd4-1 and Nedd4-2 in astrocytes regulate neuronal network function through Kir4.1 and

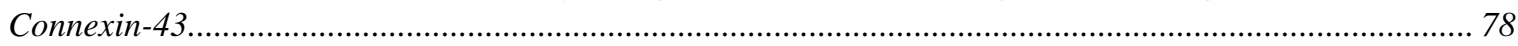

4.1.6 Regulation of Prr7 by Nedd4-2 might play a role in spine morphology.......................................... 80

4.2 FUNCTION OF WWP1 AND WWP2 IN THE BRAIN.......................................................................... 80

4.2.1 WWP1 and WWP2 are negative regulators of neurite branching ................................................ 80

4.2.2 Ubiquitination of VCP by WWP1 and WWP2 might play a role in dendritic spine formation......... 82

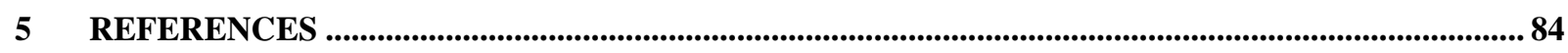



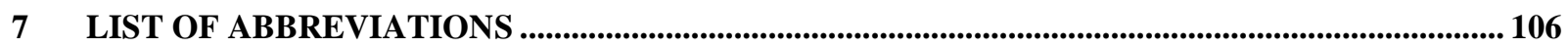

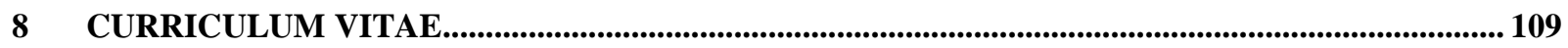

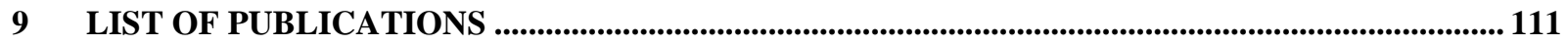




\section{SUMMARY}

Protein ubiquitination is one of the core regulatory post-translational modification in neuronal development. In this study, we used brain specific KO mice to identify novel substrate proteins of several Nedd4 family E3 ligases, i.e. Nedd4-1, Nedd4-2, WWP1 and WWP2. We developed a highly reliable approach to identify transmembrane substrate proteins of Nedd4-1 and Nedd4-2 by combining iTRAQ quantitative mass spectrometry with synaptic membrane purification. We showed that levels of the main inwardly rectifier potassium channel in astrocytes, Kir4.1, and the main gap junction protein in astrocytes, Connexin-43, are upregulated in Nedd4-1 and Nedd4-2 brain specific double KO mice. Furthermore, we showed that Kir4.1 is ubiquitinated in vivo and in vitro by Nedd4-1 and Nedd4-2 whereas Connexin-43 is ubiquitinated by Nedd4-2 in vivo via a K63-linked polyubiquitin chains. In addition, we showed that glial loss of Nedd4-1 and Nedd4-2 leads to the reduced averaged power of gamma oscillatory activity in the CA3 region of the hippocampus in Nedd4-1; Nedd4-2 double KO and Nedd4-2 single KO as compared control mice, indicating that Nedd4-1 and Nedd4-2 play a crucial role in the regulation of neuronal network through astrocytic network in the hippocampus. Moreover, we showed that Prr7 is a novel substrate of Nedd4-2 at postsynapses and Nedd4-2 conjugates K63linked polyubiquitin chains to cytoplasmic region of Prr7. Such ubiquitination of Prr7 might play a role in spine maturation during the development. Additionally, we identified CAPZA1, VCP and PKM2 as the novel substrate proteins of WWP1 and WWP2 E3 ligases. We showed that genetic deletion of WWP1 and WWP2 conditionally in neurons leads to enhanced dendrite growth in hippocampal neurons, indicating that WWP1 and WWP2 are negative regulator of dendrite development. Overexpression of CAPZA1 in wild type hippocampal neurons phenocopies the enhanced dendrite growth in WWP1; WWP2 double knockout neurons indicating that ubiquitination of CAPZA1 by WWP1 and WWP2 might play a crucial role in regulation of CAPZA1 activity thereby regulation of dendrite development. 


\section{INTRODUCTION}

The brain is the most complex human organ, perceiving all of the information from the environment, processing this information, and storing it as memories. The human brain has approximately $10^{8}$ neurons, each of which is connected to other neurons through a specialized subcellular compartment called the synapse. Neurites extending from the neuronal cell body provide the platform for synapse formation. Thus, neurite morphology is critical for determining the framework of neuronal connectivity. Because synaptic transmission is the temporal limiting factor for information transfer in the brain, studies on the development and the function of synapses and neurites are of particular importance for our understanding of brain action. The development and the function of neurons are regulated by numerous intracellular signaling pathways, which involve, for example, protein and lipid phosphorylation, calcium influx, and cyclic nucleotides. In this thesis, I describe how specific protein ubiquitination pathways regulate brain development and function.

\subsection{Ubiquitination}

Ubiquitination is a posttranslational modification by which a substrate protein is conjugated with one or more ubiquitin moieties. Ubiquitin is a protein composed of 76 amino acids with a molecular weight of approximately $8.5 \mathrm{kDa}$. Ubiquitination is achieved by a sequential reaction involving three classes of enzymes: ubiquitin-activating enzymes (E1), ubiquitin-conjugating enzymes (E2), and ubiquitin ligases (E3). In the first step, ubiquitin is activated at the expense of ATP hydrolysis by an E1 enzyme and linked with a high-energy thioester bond to the cysteine residue on an E1 enzyme. In the second step, ubiquitin is transferred to an E2 enzyme that directly interacts with an E3 enzyme. In the last step, the E3 enzyme assists with the transfer of the ubiquitin moiety onto a target protein through an isopeptide bond between the $\varepsilon$-amino group of the lysine residue on the substrate and the carboxyl group of the glycine at the C-terminus of the ubiquitin protein (Figure 1-1A-C). Based on their mode of action, E3 ligases fall into two families: Really Interesting New Gene type E3

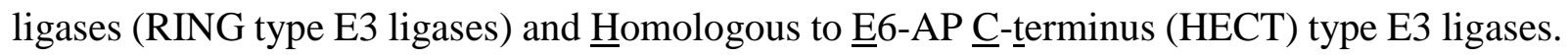
RING type E3 ligases form protein complexes with an E2-ubiquitin conjugate and the substrate. By leading the ubiquitin moiety into molecular proximity of the substrate protein, RING-type 
E3 ligases facilitate the transfer of ubiquitin onto a substrate protein. In HECT type E3 ligases, on the other hand, ubiquitin is transferred from an E2 enzyme to the cysteine residue on the catalytic HECT domain. The thioester bond between ubiquitin and the HECT domain is transient and the ubiquitin moiety is transferred to the substrate, forming a complex with the HECT type E3 ligase. Thus, the RING type E3 ligases act as a scaffold protein to bridge the E2 ligase and the substrate to facilitate ubiquitination while the HECT type E3 ligases act as a ubiquitin acceptor and then transfer it to their substrates. In vertebrates, E3 ligases are highly diverse. While only two E1 enzymes and thirty-seven E2 enzymes are encoded by the human genome, more than 600 genes encode vertebrate E3 ligases, of which 28 belong to the HECT type E3 ligase family (Komander, 2009; Rotin and Kumar, 2009). All E3 ligases form complexes with their cognate substrate proteins, so that E3 ligases are the main determinants of substrate specificity in ubiquitination.

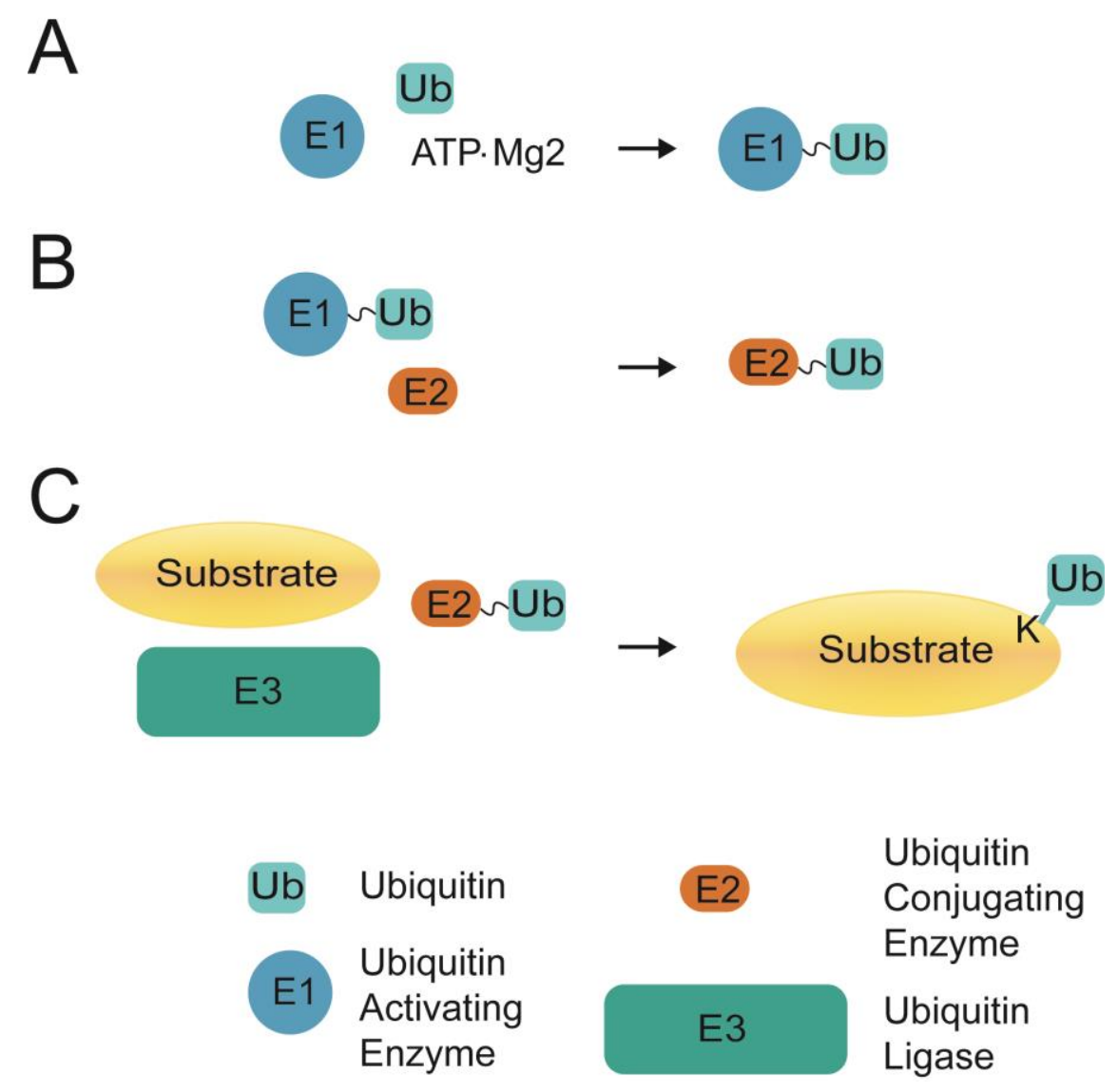

\section{Figure 1-1. Mechanism of protein ubiquitination.}

Protein ubiquitination includes 3 sequential reactions. (A) First, ubiquitin is activated in an ATPdependent manner by a ubiquitin activating enzyme (E1). Ubiquitin forms a thioester bond on a 
cysteine residue of the E1 enzyme. (B) Activated ubiquitin is transferred to a cysteine residue on a ubiquitin conjugating enzyme (E2), which is recognized by a ubiquitin ligase. (C) Ubiquitin ligase (E3 or E3 ligase) facilitates the transfer of ubiquitin from E2 to the substrate protein. Substrate specificity of ubiquitination is mainly defined by E3 enzymes.

\subsubsection{Forms of Ubiquitination}

A single ubiquitin moiety can be conjugated to a defined lysine residue (monoubiquitination) or to multiple lysine residues (multimonoubiquitination) of a substrate protein. Moreover, ubiquitination can occur in the form of polyubiquitin chains. Chain formation is achieved by the modification of the $\mathrm{N}$-terminal methionine residue on ubiquitin or by one of seven lysine residues on ubiquitin (K6, K11, K27, K29, K33, K48, K63: Figure 1-3AB). All seven lysine residues on ubiquitin can be processed in vivo to form different types of polyubiquitin chains, which then may have different functional consequences on the target protein (Figure 1-2C). For instance, K48 polyubiquitin chain formation leads a substrate protein to proteasomal degradation, whereas mono- or multimonoubiquination leads to endocytosis of plasma membrane proteins. K63 polyubiquitin chains play crucial roles in the NF-kappaBmediated signaling pathway, DNA damage response, and endocytosis and lysosomal degradation of substrate proteins. K11 polyubiquitin chains are important for cell cycle regulation and endoplasmic reticulum-associated degradation (ERAD), while K29 polyubiquitin chains are involved in lysosomal degradation and the regulation of protein functions (Ikeda and Dikic, 2008; Komander, 2009). Mass spectrometric analysis of S. cerevisiae revealed that 29\% of polyubiquitin chains are K48 polyubiquitin chains whereas 17\% of chains are K63 polyubiquitin chains. Surprisingly, K11 polyubiquitin chains are the second most abundant chain type making up $28 \%$ of the polyubiquitin chains, whereas K6, K27, K29 and K33 polyubiquitin chains have relative abundances of 11, 9, 3 and 3\% respectively (Xu et al., 2009). Recent studies have shown that mouse and rat brains have a relatively high abundance of K63 and K48 polyubiquitin chains, $\sim 55 \%$ and $\sim 30 \%$ respectively, indicating that lysosomal degradation of ubiquitinated transmembrane proteins may be as important as proteasomal degradation for neuronal development and function (Dammer et al., 2011). 


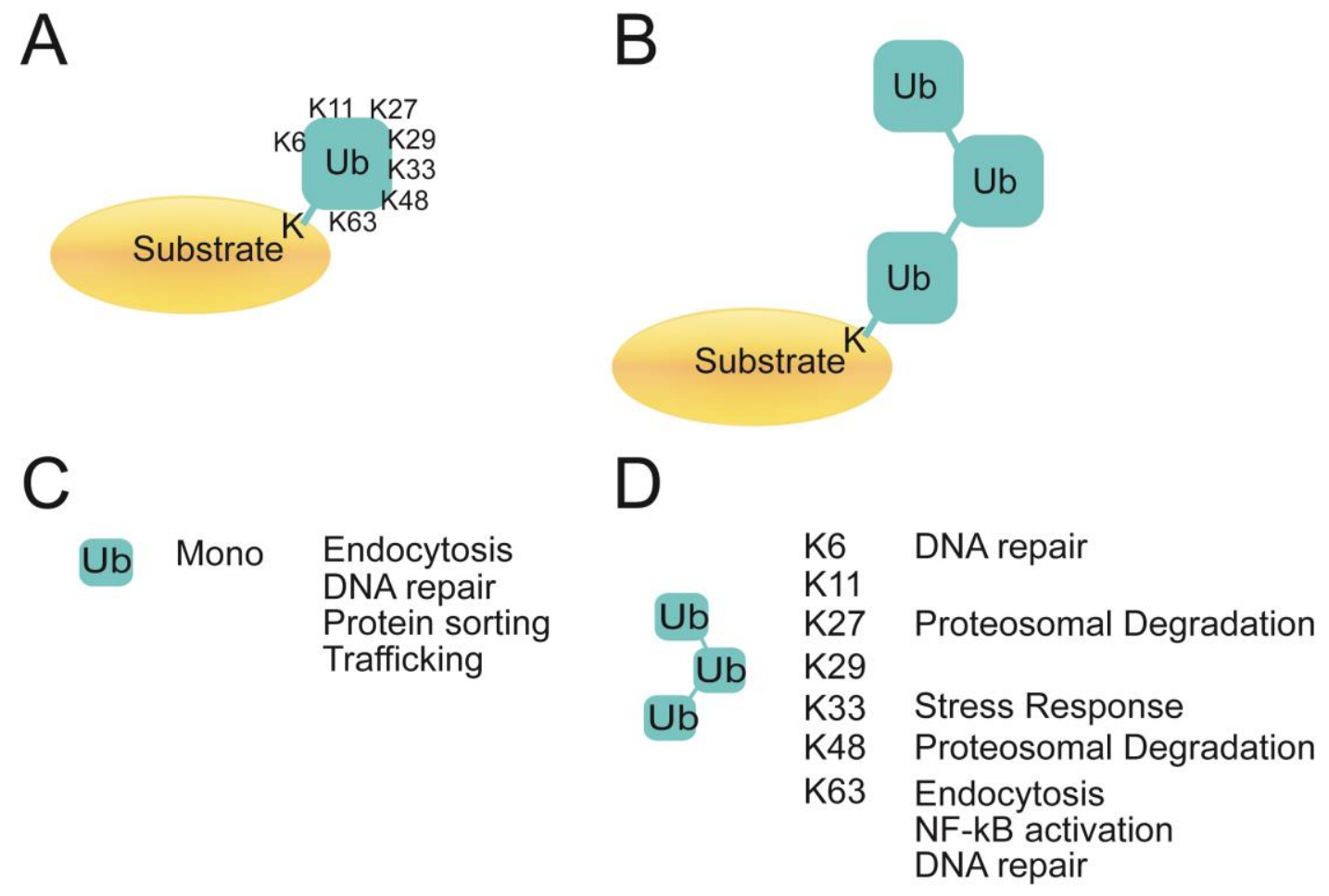

Figure 1-2. Forms of ubiquitination

(A) A single ubiquitin is conjugated on a defined lysine residue of a specific substrate protein (monoubiquitination). Conjugated ubiquitin can act as a ubiquitin acceptor for chain formation. Ubiquitin chains can be formed by modification of lysine residues at the position of K6, K11, $\mathrm{K} 27, \mathrm{~K} 29, \mathrm{~K} 33$, K48, K63 on ubiquitin. (B) Ubiquitin chains are formed in a lysine specific manner. (C) Different forms of ubiquitination have different functional consequences in the cell. Monoubiquitination is involved in endocytosis of a substrate protein, DNA repair, protein sorting and trafficking. K6-linked polyubiquitin chains are involved in DNA repair mechanism while K11-, K27- and K48-linked chains are involved in proteosomal degradation. K33-linked polyubiquitin chains are involved in stress responses, while K63-linked polyubiquitin chains play roles in endocytosis, protein-protein interactions and DNA repair process.

\subsubsection{HECT type E3 ligases}

HECT type E3 ligases are composed of 28 members with a common catalytic HECT domains at their C-termini. The HECT domain is a bilobal domain with approximately 350 amino acids. The $\mathrm{N}$-terminal part of the HECT domain is known as the $\mathrm{N}$ lobe and is responsible for the interaction of the E3 ligase with an E2 enzyme, whereas the C-terminus, called the $\mathrm{C}$ lobe, accepts the ubiquitin moiety on its catalytic cysteine residue (Figure 1-3B). Structural studies on the HECT domain of the human papilloma virus E6-associated protein (E6AP) revealed that the $\mathrm{N}$ and $\mathrm{C}$ lobes are bridged with a flexible hinge loop (Huang, 1999). The chain type specificity of HECT type E3 ligases is dependent on the structure of the C-terminus of the 
C lobe (Kim and Huibregtse, 2009; Maspero et al., 2013). Based on the similarities in the amino acid sequences, the HECT type E3 ligase superfamily is further divided into three subfamilies. The Nedd4 family has nine members, the HERC family has six members, and the other HECT type E3 ligases have thirteen members (Rotin and Kumar, 2009).

\subsubsection{Role of Nedd4 family $E 3$ ligases in the brain}

The members of the Nedd4 family of E3 ligases have a common domain structure that includes a $\mathrm{C} 2$ domain at the $\mathrm{N}$ terminus, two to four WW domains, and a HECT domain at the $\mathrm{C}$ terminus (Figure 1-3A). The $\mathrm{C} 2$ domain binds to phospholipids in a calcium-dependent manner and determines the localization of the E3 ligase within the cell (Dunn et al., 2004; Plant et al., 1997). The C2 domain also plays a regulatory role in autoinhibition by interacting with HECT domain on the same protein (Wiesner et al., 2007). The WW domain is named due to two conserved tryptophan residues and is composed of approximately 35 amino acids. In the Nedd4 family of E3 ligases, the WW domains mainly mediate substrate interaction and recognize PPXY, PPXYXXL, and LPXY motifs on substrates, where $X$ can be any amino acid (Ingham et al., 2004). However, it has been reported that the $\mathrm{C} 2$ domain can also be involved in substrate recognition. The $\mathrm{C} 2$ domain of Smurf1 directly interacts with RhoA and this interaction is necessary for the ubiquitination of RhoA by Smurf1 (Tian et al., 2011).

Based on their homologies, the members of the Nedd4 family of E3 ligases fall into four subfamilies: the Nedd4 subfamily (Nedd4-1 and Nedd4-2), the WWP subfamily (WWP1, WWP2 and Itch), the Smurf subfamily (Smurf1 and Smurf2), and the NeddL subfamily (NeddL1, NeddL2) (Figure 1-3C: Scheffner and Kumar, 2014). Nedd4-1 acts as a crucial,

positive regulator of dendrite branching by conjugating monoubiquitin to Rap2A (Kawabe et al., 2010), and is also important for the regulation of axonal growth in hippocampal neurons downstream of PTEN (Hsia et al., 2014). Finally, Nedd4-2 has been reported as a regulator of several voltage-gated sodium and potassium channels (Arevalo, 2015). 
A



B

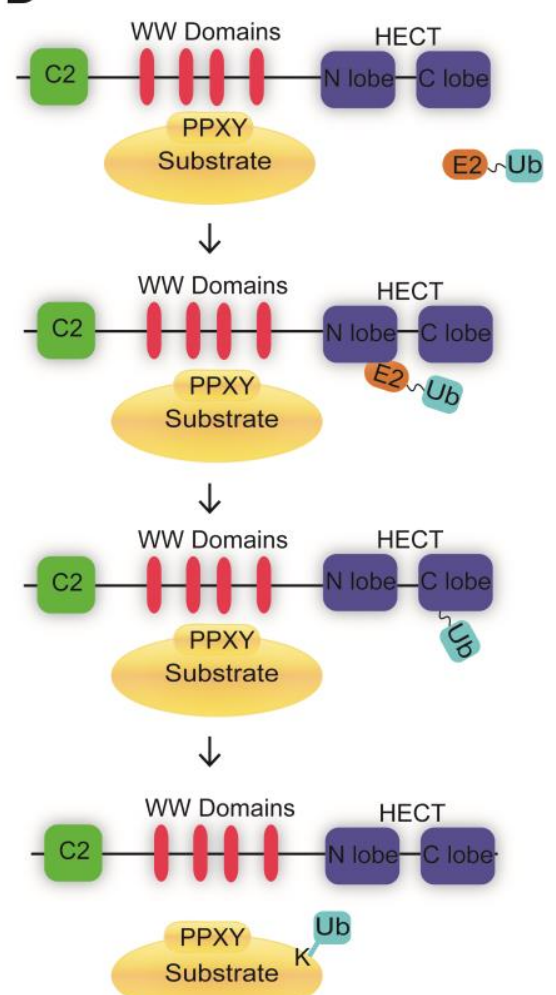

C

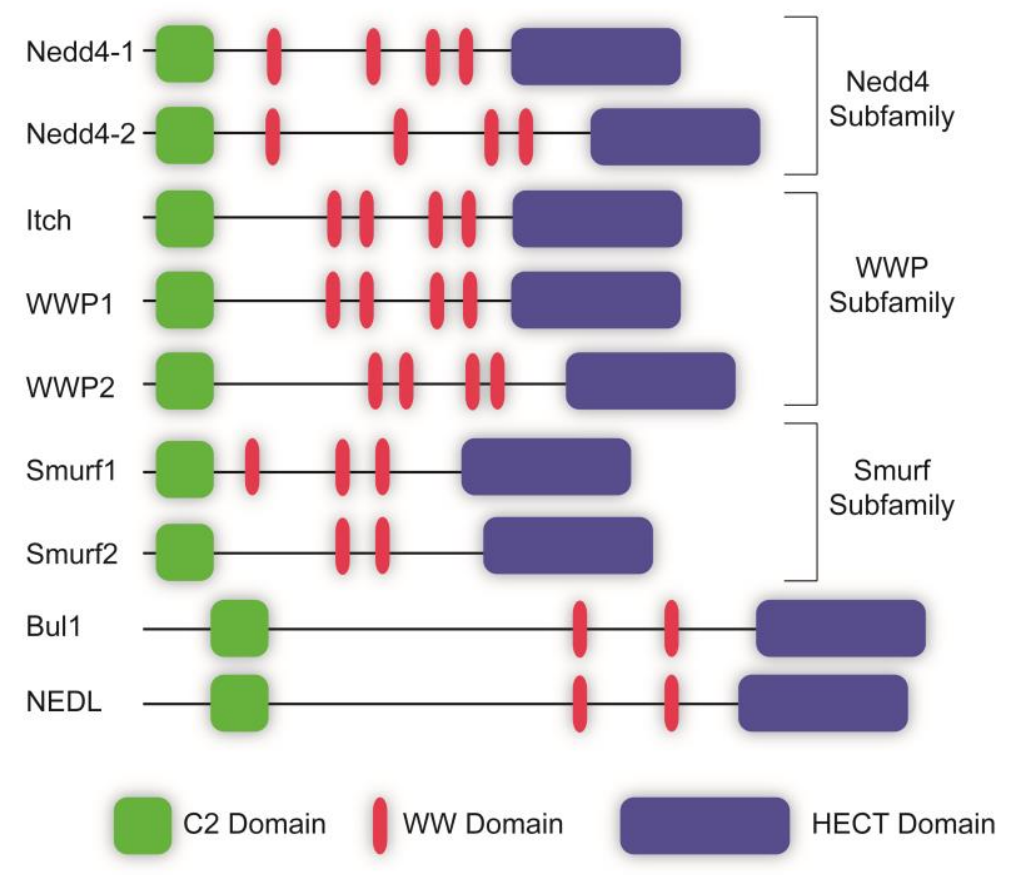

Figure 1-3. Structure and ubiquitination mechanism of Nedd4 lamily HECT-type E3 ligases

(A) Main structure of Nedd4 family E3 ligases. Nedd4 family HECT-type E3 ligases have a conserved domain structure consisting of an N-terminal C2 domain followed by 2 to $4 \mathrm{WW}$ domains, and a C-terminal HECT domain. The $\mathrm{C} 2$ domain is responsible for autoinhibition and localization of the ligase protein, while WW domains are involved in substrate recognition. The HECT domain is responsible for the catalysis of ubiquitination. (B) Ubiquitination mechanism of Nedd4 family E3 ligases. Substrate protein is recruited by the interaction of WW domains of the E3 ligase and a PPXY motif in the substrate protein. The N-lobe, the N-terminal region of the HECT domain, interacts with E2 enzyme conjugated to ubiquitin. Subsequently, ubiquitin is transferred from the $\mathrm{E} 2$ enzyme to the C-terminal region of the HECT domain, $\mathrm{C}$ lobe. Next, ubiquitin is conjugated to a defined lysine residue on the substrate protein. (C) Subfamily of Nedd4 family E3 ligases. According to their homologies, Nedd4 family E3 ligases are grouped into 4 subfamilies; Nedd4 subfamily, WWP subfamily, Smurf Subfamily, NEDL subfamily (NEDL, Bul1). 


\subsection{Development of Neuronal Cells}

In the mammalian embryonic brain, cortical neurons differentiate and develop in four partially overlapping stages. In the first stage, neurogenesis, neuronal progenitor cells generate immature neurons. This process is followed by the second stage, neuronal migration. During and after this migration, in the stage of neuritogenesis, the axons and dendrites of neurons extend and branch, providing the framework for the fourth stage, synapse formation (Figure 1-4: Kawabe and Brose, 2011).

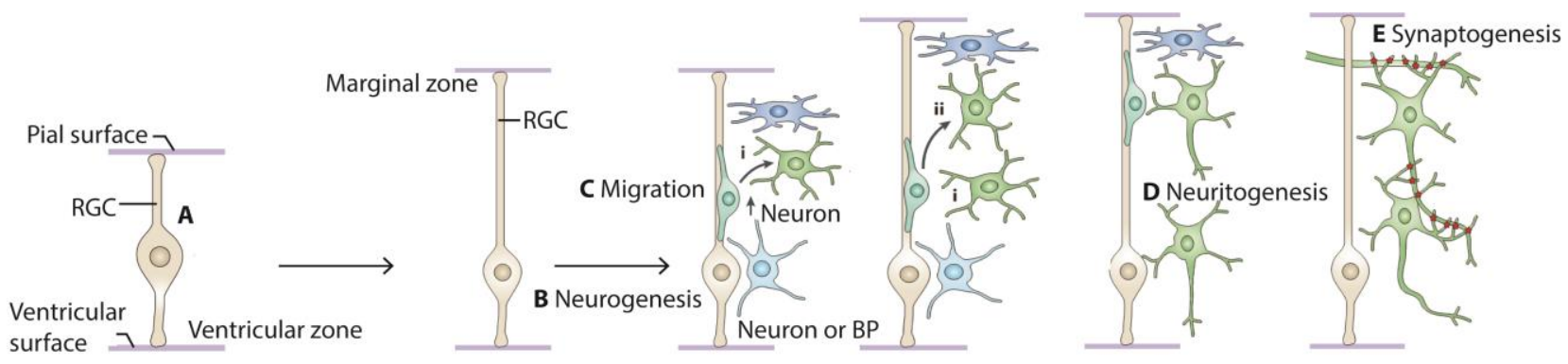

Figure 1-4. Main steps of neuronal development in mammalian brain.

(A) After neural tube closure, neuroepithelial cells divide and differentiate to radial glial cells (RGCs). The RGCs proliferate and enlarge their population. (B) RGCs give rise to premature neurons or basal progenitor cells upon asymmetric division (C) Neurons migrate along the RGC processes and termination of migration is regulated by extracellular cues from Catjal-Retzius cells which are generated in the very early phase of neurogenesis. (i) Upon receiving the termination signal, migrating newborn neurons detach from RGCs and distribute in the proper cortical layer. (ii) Neurons generated at a later stage of development migrate further towards to marginal zone. (D) Neurons in appropriate cortical layers establish their polarity and generate neurites which are specified later into axon and dendrites. (E) Neurons form synapses by synaptogenesis and synapse elimination and integrate into neuronal networks. Adapted by permission from Macmillan Publishers Ltd: Nat. Rev. Neurosci. License number 3693560392918, Copyright, 2011 (Kawabe and Brose, 2011).

\subsubsection{Neurogenesis}

In the developing neocortex, neurons originate from three types of neurogenic progenitor cells: neuroepithelial cells, radial glial cells (RGCs), and basal progenitors (BPs). Before neurogenesis, the neural tube is composed of a single type of cells called neuroepithelial cells, which undergo symmetric and proliferative cell divisions to increase the number of epithelial cells and to form the neuroepithelium. The neuroepithelium is a pseudostratified epithelial tissue due to the interkinetic nuclear migration along the apico-basal axis during symmetric cell 
division. Neuroepithelial cells are connected to each other through tight junctions and adherens junctions. Like most epithelial cells, these cells are highly polarized along their apico-basal axis. As development proceeds, neuroepithelial cells divide asymmetrically to generate neurons, forming a tissue with multiple cell layers. The layer facing the ventricle is referred to as the ventricular zone (VZ). After the onset of neurogenesis, neuroepithelial cells can give rise to RGCs upon asymmetric, differentiative divisions (Götz and Huttner, 2005).

RGCs are more fate-restricted progenitors than neuroepithelial cells and subsequently replace neuroepithelial cells during the development. In contrast to neuroepithelial cells, RGCs are connected to each other through adherens junctions without functional tight junctions, and they exhibit several astroglial properties. Although apico-basal polarity in RGCs is less prominent than in neuroepithelial cells, RGCs share important features with neuroepithelial cells, including an apical surface rich in cilia and interkinetic nuclear migration. RGCs can generate neurons by either symmetric, neurogenic division or an asymmetric division to form one neuron and one RGC. Through asymmetric cell division, RCGs and neuroepithelial cells can generate another type of progenitor cells, BPs. As the name indicates, BPs divide once to generate two neurons at the basal side of the VZ, while neuroepithelial cells and RGCs undergo neurogenic cell division at the apical side of VZ. Subsequent to migration of their nuclei to the basal side of the VZ, basal progenitors retract their processes from the apical surface. In the later stage of the development, at the basal side of VZ, BPs form a mitotic cell layer called the subventricular zone (SVZ). BPs in the SVZ differ from the neuroepithelial cells and the RGCs with their specific gene expression patterns. Some differentially expressed genes include the non-coding RNA, SVET1, and the transcription factors TBR2, CUX1, and CUX2.

\subsubsection{Neuronal Migration}

In the developing cortex, neurons originating from the progenitor cells migrate radially along the projections of radial glial cells (from the VZ or the SVZ towards the cortical plate) to their final destination. Neuronal migration is regulated by numerous intracellular and extracellular cues. Reelin is an extracellular protein secreted from Cajal-Retzuis cells at the marginal zone (MZ) of the developing cortex, forming a gradient of Reelin that decreases in concentration from the MZ to the VZ (Ogawa and Miyata, 1995). Transmembrane receptors such as very-low density lipoprotein receptor (VLDLR), low-density lipoprotein receptor 
related protein 8 (also known as apolipoprotein E receptor 2, APOER2), and photocadherins interact with Reelin, triggering intracellular signaling cascades (Arcangelo et al., 1999; Senzaki et al., 1999). Upon Reelin binding to receptors, SRC family tyrosine kinases, SRC or FYN, phosphorylate the adaptor protein Disabled Homologue 1 (DAB1), which interacts with several signaling proteins (Hiesberger et al., 1999; Trommsdorff et al., 1999). Phosphorylated DAB1 interacts with (CRK) and CRK-like (CRKL) proteins, leading to the activation of $\mathrm{C} 3 \mathrm{G}$, a guanine nucleotide exchange factor for the small GTPase RAP1. Activation of RAP1 mediates the activation of integrin, resulting in the adhesion of neurons to fibronectin in the $\mathrm{MZ}$ and the termination of migration (Sekine et al., 2012). Phosphorylated DAB1 is also involved in the formation of the dynein motor complex, composed of lissencephaly type 1 (LIS1), distribution protein nude-like 1 (NUDEL) and cytoplasmic dynein 2 heavy chain 1 . This complex affects microtubule organization, thereby playing an important role in arresting neuronal migration by arresting nucleokinesis (Kawabe and Brose, 2011; Sasaki et al., 2000). Therefore, the Reelin gradient from the $\mathrm{MZ}$ to the $\mathrm{VZ}$ supports the migrating neurons to form an inside-out pattern. Early-born neurons stop migrating shortly after starting their migration, staying in the deep cortical layers of the mature cortex, while late-born neurons migrate to reach and get ahead of early-born neurons, and form the surface layers of the cortex (Lambert de Rouvroit and Goffinet, 2001).

\subsubsection{Neuritogenesis; Axon and Dendrite Growth}

Neurons have a highly polarized structure with one long axon and multiple highly branched dendrites. The dendrites of many neurons harbor numerous synaptic spines rich in neurotransmitter receptors. Upon binding of neurotransmitters to the channel type receptors, channels open and allow sodium or calcium ions to pass from the extracellular space into the cytoplasm, causing depolarization of the membrane potential. Upon the membrane potential reaching its threshold, neurons fire an action potential. Myelinated axons conduct action potentials with a high speed to the presynaptic terminals, where neurotransmitters are released. Neuronal polarity is essential for this flow of electrical and chemical signals. Neurons develop axons and dendrites in three stages. Before starting radial migration, newborn neurons at the VZ have a multipolar structure with several immature neurites. Upon attaching to the RGCs, they convert to a bipolar spindle-like shape with the leading process directed towards the direction 
of the migration and the trailing process directed to the VZ. In the final stage, the neuron detaches from the RGC, and the leading process develops into a highly branched dendrites while the trailing process acquires axon characteristics, such as the accumulation of voltage dependent sodium channels at the proximal axon initial segment, fast conductance by being myelinated, and assembly of neurotransmitter release machinery at presynaptic terminals (Barnes and Polleux, 2009).

Extension of neurites involves three main molecular pathways: (1) the local activation of signaling molecules that provide the accumulation of new plasma membrane by vesicle transport and fusion, (2) microtubule assembly, and (3) an alteration in the dynamics of the actin cytoskeleton (Arimura and Kaibuchi, 2007a). These molecular pathways are tightly regulated by extracellular cues. Extracellular cues that affect neurite growth include secreted chemoattractants and chemorepellants [e.g. Netrin, WNT, nerve growth factor (NGF), brainderived neurotrophic factor (BDNF), and neurotrophin 3 (NT3)], membrane anchored or transmembrane ligands [e.g. neuron-glia cell adhesion molecule ( $\mathrm{NgCAM}$ ), semaphorines, ephrins, and slit], and extracellular matrix proteins (e.g. laminin) (Arimura and Kaibuchi, 2007a; O'donnell et al., 2009). Stimulation of neurons with neurotrophic factors such as BDNF or NT3 leads to the activation of PI3K, which converts phosphatidylinositol 4,5-biphosphate $\left(\operatorname{Ptd} \operatorname{Ins}(3,4,5) \mathrm{P}_{2}\right)$ to phophotidylinositol-3,4,5-triphospahte $\left(\operatorname{Ptd} \operatorname{Ins}(3,4,5) \mathrm{P}_{3}\right)$ at the tips of neurites (Schwamborn and Püschel, 2004; Shi et al., 2003; Yamada et al., 1997). PtdIns(3,4,5)P 3 recruits PDK1 to the plasma membrane, where PDK1 phosphorylates AKT1, resulting in inactivation of GSK3 $\beta$ (Burgering and Coffer, 1995; Jiang et al., 2005). In most cases, the recruitment of signaling molecules in the axon is established through active transport mechanisms involving kinesins and cargo receptors (Arimura and Kaibuchi, 2007b; Shi et al., 2004). However, within the neurite that develops into an axon, the enrichment of AKT1 is achieved by the proteasomal degradation of AKT1 in the dendrites (Yan et al., 2006). The disruption of the polarized localization of AKT1 by proteosomal inhibitors results in an increase in the number of axons projected from a single neuron.

Actin filaments (F-actins) are important for bud formation, where the polarity of growth depends on the orientation of F-actin. Organization of the actin cytoskeleton is regulated by several small GTPases, including the negative regulator, RhoA, and the positive regulators, Rac1 and Cdc42 (Puram and Bonni, 2013; Scott and Luo, 2001). RhoA activates Rho-associated 
kinase (ROK) which phosphorylates and controls myosin light chains and actomyosin contractility (Kimura et al., 1996; Mulder et al., 2004; Winter et al., 2001). Rac1 and Cdc42 activate an actin-binding protein complex called the Arp2/3 complex through their effector proteins, WASP, N-WASP, Scar/WAVE1, Scar/WAVE2 and Scar/WAVE3 (Goley and Welch, 2006). The Arp $2 / 3$ complex interacts with the side of an existing F-actin (mother filament), where it promotes the nucleation of another F-actin (daughter filament) to form a branch of Factin. Such branched F-actin often appears at the leading edge of migrating cells or at the tip of extending neurites (Jan and Jan, 2011; Nicholson-Dykstra et al., 2005). Newly formed branched actin filaments generate a force from the actin cytoskeleton to the adjacent plasma membrane, triggering the formation and extension of neurites. The generation and maintenance of this force are regulated by several actin-binding proteins, such as profilin, capping proteins, and ADF/cofilin. When actin monomers (G-actin) assemble into F-actin, G-actin is added faster to one end of the filament known as the 'barbed' end than to the other end known as the 'pointed' end. Profilin binds to G-actin and inhibits its spontaneous nucleation. In addition, Gactin/profilin complexes are only added to barbed end, limiting elongation effectively to the barbed end. ADF/cofilin elevates the rate of depolymerization and actin monomer recycling (Carlier et al., 1997; Svitkina and Borisy, 1999). The capping proteins, on the other hand, bind to the end of actin filaments and prevent further elongation. However, high concentrations of barbed-end capping proteins are essential for force generation by actin cytoskeleton, because the capping of barbed ends distal from the plasma membrane limits and channels actin polymerization to the barbed ends that are in close proximity to the membrane. Thus, the temporal and spatial regulation of capping proteins plays an essential role for neurite formation (Schafer and Cooper, 1995; Vignjevic et al., 2003).

\subsection{Contribution of Astrocytes to Neuronal Networks}

The nervous system consists of two main cell types; neuron and glia (Virchow, 1856). In vertebrates, glial cells within the central nervous system (CNS) can be categorized as astrocytes, oligodendrocytes, and microglia (Corty and Freeman, 2013). As the word glia originates from the Greek word for glue, glia cells are commonly known by their supportive functions of surrounding neurons as a connective tissue, supplying nutrients and oxygen to neurons, forming myelin sheaths, insulating neurons, protecting neurons from pathogens, and 
cleaning up dead cells within the nervous system (Verkhratsky and Butt, 2013). However, after the pioneering study by Kuffler and Potter (Kuffler and Potter, 1964), the idea of glia function not being limited to support roles within the nervous system has emerged and subsequent studies have focused on other glial functions. Advanced techniques in genetics, imaging, and electrophysiology have greatly expanded our knowledge of the essential roles of glia in regulating neuronal stem cell proliferation and precursor differentiation (Ables and DrummondBarbosa, 2011; Callan et al., 2012; Chell and Brand, 2010), neuronal migration, axon guidance (Chotard and Salecker, 2004; Rakic, 1971; Shapiro et al., 2005), and programmed cell death during the development (Marín-Teva et al., 2004). In recent years, astrocytes were shown to play key roles in the formation of both excitatory and inhibitory synapses (Christopherson et al., 2005; Elmariah et al., 2005; Mauch et al., 2001), in synapse maturation (Allen et al., 2012; Benediktsson et al., 2005; Haber et al., 2006), and in the regulation of synapse function by secretion- or contact-dependent signal transduction pathways. Thus, astrocytes have direct effects on synapse number, synapse strength and the function of neuronal circuits (Barker and Ullian, 2010).

\subsubsection{Potassium Spatial Buffering}

In the 1990s, the concept of the 'tripartite synapse' emerged. Several lines of evidence revealed the presence of bidirectional communication between astrocytes and neurons. Astrocytes obtain information from synaptic elements and they respond to synaptic activity, contributing to neuronal networks. In fact, astrocytes release various neuroactive substances such as D-serine, ATP, or neurotrophic factors when they are depolarized or stimulated at certain receptors (mGluR2 or mGluR5) (Coco et al., 2003; Mothet et al., 2005). These substances act on their receptors to modulate synaptic activity (Newman, 2003; Panatier et al., 2006). Thus, brain function depends on the activity of a neuron-glia network rather than on the neuronal network alone (Olsen and Sontheimer, 2008).

Following the onset of the action potential, the neuronal membrane potential repolarizes by an efflux of potassium, which causes a transient increase in the local extracellular potassium concentration. Despite such a transient elevation, the extracellular potassium concentration is maintained at approximately $3 \mathrm{mM}$, independent from the concentration changes in blood serum levels (Katzman, 1976; Somjen, 1979). In the tripartite synapse, one of the main functions of 
astrocytes is the clearance of excess potassium in the extracellular space, a function known as potassium spatial buffering (Orkand et al., 1966). Within the CNS, astrocytes are characterized by their highly selective membrane permeability to potassium and their strong negative resting membrane potential (Kuffler, 1967; Ransom and Goldring, 1973). Electrophysiological studies demonstrated that these two unique features of astrocytes arise from inwardly rectifying channels (Kir) expressed specifically within astrocytes (Kofuji and Newman, 2004). The family of Kir genes is composed of 16 members, sharing a common membrane topology with two transmembrane domains and cytoplasmic $\mathrm{N}$ and $\mathrm{C}$ terminals. Each channel has four subunits that assemble as homomultimers or heteromultimers. The Kir channel family is divided into seven subfamilies, which are named Kir1.x-Kir7.x (Nichols and Lopatin, 1997). Although 16 different subunits exist, all Kir channels exhibit several common features. First, they preferentially conduct potassium toward the inside of the cell in spite of also having some outward currents. Second, the potassium current increases with a high extracellular potassium concentration. Finally, the currents are blocked by micromolar concentration of $\mathrm{Ba}^{2+}(\mathrm{Olsen}$ and Sontheimer, 2008).

Although several Kir channel subunits have been identified in astrocytes, numerous studies revealed that Kir4.1, encoded by KCJN10 gene, is the main subunit of astrocytic Kir channels. Indeed, knock-out studies have shown that in the absence of Kir4.1, astrocytes show a dramatic reduction of potassium permeability, depolarized resting membrane potential, and the lack of $\mathrm{Ba}^{2+}$ sensitive currents (Djukic et al., 2007; Neusch et al., 2006; Olsen et al., 2006). These results are supported by studies showing that siRNA-mediated knockdown of Kir4.1 in the spinal cord or cortical astrocytes results in the complete loss of $\mathrm{Ba}^{2+}$ sensitive currents, a 5fold increase in input resistance (i.e a decrease in the resting ion permeability), and a depolarized resting membrane potential (Kucheryavykh et al., 2007; Olsen et al., 2006).

Kir4.1 contributes to another important functional aspect of astrocytes within tripartite synapses: the uptake of glutamate released from presynaptic neurons (Anderson and Swanson, 2000). Glutamate uptake by astrocytes mainly relies on the activity of two glutamate transporters, GLT1 and GLAST. These transporters function in an electrogenic fashion and the uptake of glutamate is more favorable at a negative resting membrane potential (Danbolt, 2001). The expression of Kir4.1 can affect the glutamate uptake through these transporters, because Kir4.1 sets the membrane potential of astrocytes to around $-85 \mathrm{mV}$. Indeed Kir4.1 knock-down 
using siRNA or blocking functional Kir4.1 by $\mathrm{Ba}^{2+}$ results in a $30 \%$ reduction of glutamate uptake via astrocytic GLT1 transporters (Kucheryavykh et al., 2007). Similar results were also reported in brain specific Kir4.1 conditional knockout mice. During Schaffer collateral stimulation, whole cell currents of astrocytes are mainly dependent on potassium influx through Kir channels and the uptake of glutamate by GLT1. Upon application of $\mathrm{Ba}^{2+}$ and/or DL-threo$\beta$-benzyloxyaspartic acid (a nonselective GLT1 inhibitor), whole cell current within astrocytes can be studied by Schaffer collateral stimulation. GLT1 generated peak currents in astrocytes upon Schaffer collateral stimulation was reduced by $50 \%$ in brain-specific Kir4.1 conditional knockout mice, indicating a critical role of Kir4.1 in the glutamate uptake by astrocytes (Djukic et al., 2007).

\subsubsection{Intercellular Calcium Waves}

Since the late 1980's, it has been known that astrocytes express many ion channels and membrane receptors by which they can change their membrane potential and intracellular calcium levels, e.g. upon the activation of neurons (Barres et al., 1990; Macvicar and Tse, 1988; Marrero et al., 1989; Salm and McCarthy, 1990; Usowicz et al., 1989). It was also proposed that astrocytes transmit increased calcium levels to adjacent astrocytes through GAP junctions, thereby creating intercellular calcium waves (ICWs) along their networks in the form of rapid long-distance signal transmission with a velocity of 15-20 $\mu \mathrm{m} / \mathrm{s}$ (Charles et al., 1991; CornellBell et al., 1990). Interestingly, neuronal activity can modulate astroglial activity and vice versa. Low stimulation of neurons within the dentate gyrus of brain slices results in continuous calcium waves in astrocytes in the CA3 region (Dani et al., 1992), indicating that neuronal networks affect astrocytic function. In turn, it was also shown by utilizing both calcium imaging techniques and the dual patching of astrocytes and pyramidal neurons within the CA1 region that astrocytes are involved in the potentiation of miniature inhibitory postsynaptic currents (mIPSCs) in pyramidal neurons (Kang et al., 1998). The impact of astrocytic networks on excitatory neuronal function has been reported as spontaneous calcium increases in astrocytes, which is mediated by NMDA-dependent neuronal excitation (Parri et al., 2001). Thus, there is an extraneuronal pathway composed of the astroglial network in the brain and the reciprocal communication between neuronal and astrocytic networks jointly control the function of the brain. 
The transfer of information from neurons to astrocytes in the form of a calcium signal occurs mainly through the spillover of synaptic neurotransmitters such as glutamate or ATP. These ligands bind to G-protein coupled receptors (GPCRs) on astrocytes to activate a $\mathrm{G}_{\mathrm{q}}$ heterotrimeric G-protein. The $\mathrm{G}_{\mathrm{aq}}$ subunit of a heterotrimeric G-protein activates phospholipase $\mathrm{C}$ (PLC), resulting in the synthesis of diacylglycerol and inositol triphosphate ( $\left.\mathrm{IP}_{3}\right)$ from phosphatidylinositol biphosphate $\left(\mathrm{PIP}_{2}\right)$. $\mathrm{IP}_{3}$ then binds to its receptor on the endoplasmic reticulum, leading to the release of calcium into the cytoplasm. ICWs can be blocked by thapsigargin, an inhibitor of sarco/endoplasmic reticulum calcium ATPase, indicating that calcium release from an internal calcium store is required for ICWs (Charles et al., 1993). However, calcium increases in astrocytic cells can also be induced by calcium permeable ligandgated ionotropic receptors (e.g. glial NMDA receptors in the cerebral cortex or glial AMPA receptors in the cerebellum), activity of $\mathrm{Na}^{+} / \mathrm{Ca}^{2+}$ exchangers, or Transient Receptor Potential A1 (TRPA1) channels (Kirischuk and Ketfenmann; Matsui and Jahr, 2006; Shigetomi et al., 2011, 2013; Verkhratsky and Kirchhoff, 2007). An intracellular calcium increase in astrocytes results in the release of gliotransmitters, which can modulate neighboring glia, neurons and vascular cells (Bezzi and Volterra, 2001; Parpura et al., 1994). Upon a calcium increase, astrocytes release ATP through Connexin-43 hemichannels, and they stimulate intercellular calcium signaling through neighboring astrocytes. It has been shown, for example, that the connexin hemichannel activator quinine induces ATP release and a calcium increase in astrocytes (Stout et al., 2002).

\section{$1.4 \quad$ Neuronal Oscillations}

The human brain is composed of 100 billion neurons, each of which can fire actin potentials at $5-50 \mathrm{~Hz}$. Every neuron has 1,000 to 10,000 synapses, thereby forming a functional network, wherein synchronized activities take place (Buzsaki and Wang, 2012; Kawabe and Brose, 2011). Neuronal oscillations within a neuronal network are rhythmic and repetitive fluctuations of activity, which can be measured as periodic local field potentials using electrodes or by electrocorticography, electroencephalography or magnetoencephalography. These oscillations have been characterized as sine waves of different frequencies, peak amplitudes, and phases (Mathalon and Sohal, 2015). The temporal frequencies of natural network oscillations range from 0.5 to $200 \mathrm{~Hz}$. Based on these frequencies, they are divided into delta oscillations $(0.5-3 \mathrm{~Hz})$, theta oscillations $(3-8 \mathrm{~Hz})$, alpha oscillations $(8-13 \mathrm{~Hz})$, gamma 
oscillations (30-90 Hz), and ultrafast oscillations $(90-200 \mathrm{~Hz})$. Oscillatory activities take place in various brain regions, including the neocortex, the hippocampus, the thalamus and the olfactory bulb (Bartos et al., 2007). The oscillatory activity of neuronal networks has been correlated with higher brain functions such as perception, attention, consciousness, and memory (Basar et al., 2000).

\subsubsection{Gamma Oscillations}

Within the different frequency spectrums, gamma oscillations have mostly been related to cognitive functions such as temporal encoding, recall of information, sensory processing, and memory formation. Gamma oscillations can be detected in many cortical and subcortical areas; however, the hippocampus is the region containing one of the highest amplitudes and it is important for exploratory behavior, working memory, encoding memories, and the retrieval of memory (Bragin et al., 1995; Colgin and Moser, 2010; Fuchs et al., 2007; Hájos and Paulsen, 2009). Gamma oscillations can be induced in acute hippocampal slices in vitro by the application of agonists such as 3,5 dihydroxyphenylglycine (DHPG) for metabotropic glutamate receptors (mGluRs), carbachol for muscarinic acetylcholine receptors (mAChRs), or kainate for kainate receptors (KR; Fellous and Sejnowski, 2000; Fisahn, 2004; Fisahn et al., 1998; Whittington et al., 1995), as well as by application of high potassium solutions (LeBeau et al., 2002). The gamma oscillations induced by the individual stimuli differ from each other in terms of their dependencies on pharmacological inhibitors, indicating that multiple mechanistic pathways are involved. For example, in the CA1 region of the hippocampus, mGluR-induced gamma oscillations and kainate-induced gamma oscillations are blocked by bicuculline (a GABA receptor antagonist) but are not affected by AMPA receptor antagonists, indicating that mGluRand KR-mediated oscillations are dependent mainly on GABAergic activation (Fisahn, 2004; Whittington et al., 1995). Carbachol-induced oscillations in the CA3 region, on the other hand, are blocked by both $\mathrm{GABA}_{\mathrm{A}}$ receptor antagonists and AMPA receptor antagonists, indicating that $\mathrm{mAChR}$-mediated oscillations are dependent on both excitatory and inhibitory pathways (Fisahn et al., 1998; Mann et al., 2005). Interestingly, potassium-induced oscillations in the CA1 and the $\mathrm{CA} 3$ regions are blocked completely by $\mathrm{GABA}_{\mathrm{A}}$ receptor antagonists but are only partially inhibited by AMPA receptor blockers (LeBeau et al., 2002). These differences in the dependency of gamma oscillations on inhibitory and excitatory pathways can be explained by 
the expression profiles of receptors within the relevant networks. KRs and mGluRs are mainly expressed in interneurons, so the agonists of them are thought to activate the interneurons directly, and this occurs without assistance from glutamatergic neurons. mAChRs are mainly expressed in hippocampal pyramidal neurons and the activation of interneurons by pyramidal neurons depends on the excitation of mAChRs. In potassium induced oscillations, potassium activates both interneurons and pyramidal neurons; thus, the oscillations are partially dependent on excitation from pyramidal neurons (Bartos et al., 2007).

\subsection{Aims of the Present Study}

The present study was designed to unveil the roles of Nedd4 Family E3 ligases (Nedd41, Nedd4-2, WWP1 and WWP2) in mouse brain by identifying novel substrate proteins of these E3 ligases.

In first part of this study, we focused on the roles of Nedd4-1 and Nedd4-2 E3 ligases in mouse brain by identifying novel substrate proteins of these ligases at synapses. In the second part of this study, we focused on characterizing the roles of the WWP subfamily E3 ligases, WWP1 and WWP2, in the developing nerve cells by identifying novel substrate proteins in mouse brain homogenates. 


\section{MATERIAL and METHODS}

\subsection{Animals}

All experiments conducted on mice were performed in compliance with the guidelines for the welfare of experimental animals approved by the State Government of Lower Saxony (Niedersächsisches Landesamt für Verbraucherschutz und Lebensmittelsicherheit Permission 33.9.42502-04/103/08) and the Max Planck Society (comparable to National Institute of Health Guidelines). To obtain conditional knock-out mice, the $N e d d 4-1^{\mathrm{f} / \mathrm{f}}, N e d d 4-2^{\mathrm{f} / \mathrm{f}}, W W P 1^{\mathrm{f} / \mathrm{f}}$, and $W W P 2^{\mathrm{f} / \mathrm{f}}$ mouse lines were generated by Dr. Hiroshi Kawabe and Prof. Dr. Nils Brose. The EMX1-Cre line was generated and provided by Kevin R. Jones (Jessica A. Gorski et al. 2002). The NEX1-Cre line was generated and provided by Sandra Goebbels and Prof. Dr. Klaus A. Nave (Goebbels et al. 2006)

\section{$2.2 \quad$ Reagents}

\subsubsection{Chemicals}

Table 1. List of chemicals used in this study

\begin{tabular}{|l|l|}
\hline Product & Company \\
\hline Acrylamide/N,N-Methylene-bis-Acrylamide & National Diagnostics \\
\hline Adenosine Triphosphate & Sigma-Aldrich \\
\hline Agarose & Invitrogen \\
\hline Ammonium Persulfate & Sigma-Aldrich \\
\hline Ampicilin & Invitrogen \\
\hline Aprotinin & Roche \\
\hline Bacto-Agar & Invitrogen \\
\hline Bovine Serum Albumin (BSA), Fraction V & Thermo Scientific \\
\hline Boric acid & Sigma-Aldrich \\
\hline Bromophenol Blue & Sigma-Aldrich \\
\hline Calcium Chloride & Sigma-Aldrich \\
\hline CHAPS & Biomol Feincgemikalien \\
\hline Comassie Brillant Blue R250 & Biomol Feincgemikalien \\
\hline Cycloheximide & Sigma-Aldrich \\
\hline Dimethyl Sulfoxide (DMSO) & Sigma-Aldrich \\
\hline Dithiothreitol (DTT) & Sigma-Aldrich \\
\hline DNA Ladder Mix Sample, GeneRuler & Fermentas \\
\hline DNAase & Sigma-Aldrich \\
\hline dNTPs & GE Healthcare \\
\hline & \\
\hline
\end{tabular}




\begin{tabular}{|l|l|} 
Skim Milk & Nestle \\
\hline ECL Reagents & GE Healthcare \\
\hline Ethanol & Sigma-Aldrich \\
\hline Ethidium Bromide & Carl Roth \\
\hline Ethylenediaminetetraacetic acid (EDTA) & Sigma-Aldrich \\
\hline Fish Skin Gelatin & Sigma-Aldrich \\
\hline GelRed & Biotium \\
\hline Glucose Monohydrate & Sigma-Aldrich \\
\hline Glutathione & Sigma-Aldrich \\
\hline Glycerol 99\% & Merck \\
\hline Goat Serum & Invirogen GIBCO \\
\hline HEPES & Sigma-Aldrich \\
\hline Hydrochloric acid (HCl) & Sigma-Aldrich \\
\hline IPTG & Biomol Feincgemikalien \\
\hline Kanamycin & Invitrogen \\
\hline Leupeptin & Roche \\
\hline Lysozyme & Roche \\
\hline Luria Broth (LB) & Invitrogen \\
\hline Magnesium Chloride & Sigma-Aldrich \\
\hline Methanol & Sigma-Aldrich \\
\hline N-ethylmaleimide (NEM) & Sigma-Aldrich \\
\hline N'N'N'-Tetramethylethyl Enediamine (TEMED) & BioRad \\
\hline Photassium Chloride (KCl) & Sigma-Aldrich \\
\hline Phenymethylsulfonyl Fluoride (PMSF) & Roche \\
\hline Sodium Butyrate & Merck \\
\hline Sodium Chloride & Sigma-Aldrich \\
\hline Sodium Dodecyl Sulfate (SDS) & Roche \\
\hline Sodium Phosphate & Roche \\
\hline Sucrose & Sigma-Aldrich \\
\hline Tris Base & Sigma-Aldrich \\
\hline Triton X-100 & Roche \\
\hline Tween 20 & Sigma-Aldrich \\
\hline X-Gal & BioMol \\
\hline & \\
\hline
\end{tabular}




\subsubsection{Bacterial strains}

Table 2. List of bacteria strains used during this study

\begin{tabular}{|l|l|}
\hline E. coli XL-1 Blue Electrocompotent cells & Stratagene \\
\hline E. coli Electro10-Blue Electrocompetent cells & Stratagene \\
\hline E. coli TOP10 Electrocompotent cells & Invitrogen \\
\hline E. coli JM109 Electrocompotent cells & Promega \\
\hline E. coli BL21 Rosetta Electrocompotent cells & Stratagene \\
\hline
\end{tabular}

\subsubsection{Kits and apparatus}

Table 3. List of kits and apparatus used in this study

\begin{tabular}{|l|l|}
\hline PureLink Quick Plasmid Miniprep Kit & Invitrogen \\
\hline PureLink HiPure Plasmid Midiprep Kit & Invitrogen \\
\hline EndoFree Plasmid Maxi Kit & QIAGEN \\
\hline PureLink Gel Extraction Kit & Invitrogen \\
\hline TOPO-TA Cloning Kit & Invitrogen \\
\hline Bradford Protein Assay Kit & Bio-Rad \\
\hline
\end{tabular}

\subsubsection{Enzymes from commercial sources}

Table 4. List of commercial enzymes used in this study

\begin{tabular}{|l|l|}
\hline Alkaline Phophatase & Roche \\
\hline Cloned Pfu DNA Polymerase AD & Agilent \\
\hline DNaseI & Sigma-Aldrich \\
\hline Pfu Turbo DNA Polymerase & Agilent \\
\hline Taq Polymerase (RedTaq) & Sigma-Aldrich \\
\hline
\end{tabular}

\subsubsection{Oligonucleotides}

Oligonucleotides used in this study were synthesized in MPI-EM DNA Core Facility and listed below. 
Table 5. List of oligonucleotides used in this study (Restriction enzyme sites employed for molecular cloning are underlined. RS; Restriction sites)

\begin{tabular}{|l|l|l|}
\hline Number & Sequence (5'-3') & RS \\
\hline 30925 & 5'-TCTAGTCGACCTATACGGCTGTAGTCCTCCCGAACAAG -3' & Sal I \\
\hline 30954 & 5'-TATAGAATTCCGCCGCCGCCTCAAACGG -3' & EcoRI \\
\hline 30660 & 5'-AGGGAATTCCTTGCAAAGATTGCCCGGCC -3' & EcoRI \\
\hline 30661 & 5'-CCTCTCGAGTCAGACGTTGCTGATGCGCACAC -3' & XhoI \\
\hline
\end{tabular}

\subsubsection{Vector plasmids}

Table 6. List of vector used in this study

\begin{tabular}{|l|l|}
\hline pcDNA3.1-myc-VCP & Provided by Dr. Hemmo Meyer \\
\hline pcDNA3.2-HA-Connexin-43 & Addgene \#49851 \\
\hline pCMV2-FLAG-Nedd4-1 & Provided by Hiroshi Kawabe \\
\hline pCMV2-FLAG-Nedd4-2 & Provided by Hiroshi Kawabe \\
\hline pCMV2-FLAG-Prr7 & Provided by Dr. Yoshinori Fujiyoshi \\
\hline pCMV2b-FLAG-Kir4.1 Cyt & Generated in this study \\
\hline pCRII-TOPO & Invitrogen \\
\hline pCR2.1-TOPO-Prr7 Cyt & Generated in this study \\
\hline pCR2.1-TOPO-Kir4.1 Cyt & Generated in this study \\
\hline pDEST-hNedd4-1 & Provided by Dr. Daniela Rotin \\
\hline pDEST-hNedd4-2 & Provided by Dr. Daniela Rotin \\
\hline pET28-mE1 & Addgene \#32534 \\
\hline pEF1-CAND1 & Provided by Dr. Hiroshi Kawabe \\
\hline pEGFP-C1-Nedd4-1 & Provided by Hiroshi Kawabe \\
\hline pEGFP-C2-Nedd4-2 & Provided by Hiroshi Kawabe \\
\hline pEGFP-CMV-Kir4.1 & Provided by Dr. Johannes Hirrlinger \\
\hline pGEX4T-1 & GT Healthcare Life Sciences \\
\hline pGEX4T-1-WWP1 WT & Provided by Dr. Hiroshi Kawabe \\
\hline pGEX6P-1 & GT Healthcare Life Sciences \\
\hline pGEX6P-1-WWP2 WT & Provided by Dr. Hiroshi Kawabe \\
\hline pGEX4T-1- UbcH5b & Provided by Dr. Andrea Pichler \\
\hline pGEX6P-1- UbcH5c & Provided by Dr. Andrea Pichler \\
\hline pGEX4T-1- UbcH6 & Provided by Dr. Andrea Pichler \\
\hline pGEX4T-1- UbcH7 & Provided by Dr. Andrea Pichler \\
\hline pRaichuMyc & Provided by Dr. Hiroshi Kawabe \\
\hline pRaichuMyc-CAPZA1 & Provided by Dr. Hiroshi Kawabe \\
\hline & \\
\hline
\end{tabular}




\begin{tabular}{|l|l|} 
pRaichuMyc-Prr7 Cyt & Generated in this study \\
\hline pRaichuMyc-Pkm2 & Provided by Dr. Hiroshi Kawabe \\
\hline pQE9-p97 & Provided by Dr. Hemmo Meyer \\
\hline
\end{tabular}

\subsubsection{Antibodies}

Table 7. List of antibodies used in this study

\begin{tabular}{|l|c|c|c|c|}
\hline \multirow{2}{*}{ Antibody } & Host & \multirow{2}{*}{ Origin } & \multicolumn{2}{c|}{ Usage and Dilution } \\
\cline { 3 - 5 } & Species & Wig & ICC \\
\hline Actin (AC40) & Mouse & Sigma-Aldrich & $1: 2000$ & - \\
\hline Actin & Rabbit & Sigma-Aldrich & $1: 1000$ & - \\
\hline Nedd4-1 & Mouse & BD & $1: 500$ & - \\
\hline Nedd4-2 & Rabbit & Cell Signaling & $1: 2000$ & - \\
\hline MAP2 & Chicken & Novus & - & $1: 1000$ \\
\hline EGFP & Mouse & Roche & $1: 1000$ & $1: 1000$ \\
\hline EGFP & Rabbit & SySy & $1: 1000$ & $1: 1000$ \\
\hline PSD-95 & Mouse & BD & $1: 500$ & - \\
\hline RabGDI & Mouse & SySy & $1: 1000$ & - \\
\hline Prr7 & Mouse & Acris & $1: 1000$ & - \\
\hline Prr7 & Rabbit & from Dr. Yoshinori Fujiyoshi & $1: 2000$ & - \\
\hline Kir4.1 & Rabbit & Labome & $1: 1000$ & - \\
\hline Cnx43 & Rabbit & from Dr. Irina Majoul & $1: 2000$ & - \\
\hline c-myc & Rabbit & Sigma-Aldrich & $1: 1000$ & - \\
\hline c-myc (9E10) & Mouse & Sigma-Aldrich & $1: 1000$ & $1: 1000$ \\
\hline HA & Rabbit & Sigma-Aldrich & $1: 1000$ & - \\
\hline FLAG & Mouse & Sigma-Aldrich & $1: 1000$ & - \\
\hline FLAG & Rabbit & Cell Signaling & $1: 1000$ & - \\
\hline$\beta-$ Tubulin & Mouse & Sigma-Aldrich & $1: 10000$ & - \\
\hline Ubiquitin P4D1 & Mouse & Santa Cruz & $1: 1000$ & - \\
\hline Ubiquitin K48 & Rabbit & Cell Signaling & $1: 500$ & - \\
\hline Ubiquitin K63 & Rabbit & Cell Signaling & $1: 100$ & - \\
\hline
\end{tabular}

Table 8. List of secondary antibodies used in this study

\begin{tabular}{|c|c|c|c|c|}
\hline Antibody & $\begin{array}{c}\text { Host } \\
\text { Species }\end{array}$ & $\begin{array}{c}\text { Conjugated } \\
\text { Substrate/Dye }\end{array}$ & Origin & Usage Dilution \\
\hline $\begin{array}{c}\text { anti-Mouse } \\
\text { IgG }\end{array}$ & Goat & $\begin{array}{c}\text { Horse Radish } \\
\text { Peroksidase }\end{array}$ & BioRad & WB; $1: 10000$ \\
\hline $\begin{array}{c}\text { anti-Rabbit } \\
\text { IgG }\end{array}$ & Goat & $\begin{array}{c}\text { Horse Radish } \\
\text { Peroksidase }\end{array}$ & BioRad & WB; $1: 10000$ \\
\hline
\end{tabular}




\begin{tabular}{|c|c|c|c|c|}
$\begin{array}{c}\text { anti-Mouse } \\
\text { IgG }\end{array}$ & Goat & $\begin{array}{c}\text { IL-COR IRDye } \\
800\end{array}$ & Rockland & WB; $1: 3000$ \\
\hline $\begin{array}{c}\text { anti-Mouse } \\
\text { IgG }\end{array}$ & Goat & $\begin{array}{c}\text { IL-COR IRDye } \\
700\end{array}$ & Rockland & WB; $1: 3000$ \\
\hline $\begin{array}{c}\text { anti-Rabbit } \\
\text { IgG }\end{array}$ & Goat & $\begin{array}{c}\text { IL-COR IRDye } \\
800\end{array}$ & Rockland & WB; $1: 3000$ \\
\hline $\begin{array}{c}\text { anti-Rabbit } \\
\text { IgG }\end{array}$ & Goat & $\begin{array}{c}\text { IL-COR IRDye } \\
700\end{array}$ & Rockland & WB; $1: 3000$ \\
\hline $\begin{array}{c}\text { anti-Mouse } \\
\text { IgG }\end{array}$ & Goat & $\begin{array}{c}\text { Alexa Fluor } \\
488 / 555 / 633\end{array}$ & Invitrogen & $\begin{array}{c}\text { IHC/ICC; } \\
1: 1000\end{array}$ \\
\hline $\begin{array}{c}\text { anti-Rabbit } \\
\text { IgG }\end{array}$ & Goat & $\begin{array}{c}\text { Alexa Fluor } \\
488 / 555 / 633\end{array}$ & Invitrogen & $\begin{array}{c}\text { IHC/ICC; } \\
1: 1000\end{array}$ \\
\hline $\begin{array}{c}\text { anti-Chicken } \\
\text { IgG }\end{array}$ & Goat & $\begin{array}{c}\text { Alexa Fluor } \\
488 / 555 / 633\end{array}$ & Invitrogen & $\begin{array}{c}\text { IHC/ICC; } \\
1: 1000\end{array}$ \\
\hline
\end{tabular}

\subsection{Molecular Biology}

\subsubsection{Plasmid DNA preparation using miniprep, midiprep and endofree maxiprep}

The plasmid DNA preparation was performed using the PureLink ${ }^{\mathrm{TM}}$ Quick Plasmid Miniprep Kit, HiPure Plasmid Filter Midiprep Kit, HiPure Plasmid Maxiprep Kit (Invitrogen), or EndoFree Plasmid Maxi Kit (Qiagen). For each kit, manufacturers' instructions were followed and DNA was resuspended in TE buffer. Final concentration of the plasmid DNA was adjusted to $2 \mathrm{mg} / \mathrm{mL}$ with TE Buffer.

TE Buffer: 10 mM Tris-HCl pH 7.4 (Sigma-Aldrich), 1 mM EDTA (Sigma-Aldrich)

\subsubsection{Glycerol stocks}

$800 \mu \mathrm{L}$ of an overnight grown culture of $E$. coli electro-transformed with a construct was mixed with $200 \mu \mathrm{L}$ of $80 \%$ autoclaved glycerol, vortexed and stored at $-80^{\circ} \mathrm{C}$. To grow fresh cultures, small piece of glycerol stock was picked up using a pipette tip and transferred into LB medium with appropriate antibiotic.

\subsubsection{Plasmid transformation into bacteria}

Electroporation of electro-competent E. coli cells was carried out using Bio-Rad E. coli Pulser at $1.8 \mathrm{kV}, 25 \mu \mathrm{F}$ with pulse controller set at $200 \Omega$. Briefly, $50 \mathrm{ng}$ of plasmid DNA or 1$2 \mathrm{uL}$ of the ligation reactions (T4 Ligation or pCR2.1-TOPO Ligation) was mixed with $50 \mu \mathrm{L}$ of appropriate electro-competent $E$. coli cells thawed and kept on ice. The mixture of DNA and competent cell was transferred into pre-cooled electroporation cuvette $(0.1 \mathrm{~cm}$, Bio-Rad $)$. After 
application of the electric pulse, E. coli cells were resuspended in $800 \mu \mathrm{L}$ of LB medium and incubated for $1 \mathrm{~h}$ at $37^{\circ} \mathrm{C}$ for recovery. E. coli cells were centrifuged at $10000 \mathrm{~g}$ for $1 \mathrm{~min}$ and the pellet was resuspended in $100 \mu \mathrm{L}$ of $\mathrm{LB}$ medium and plated on the selection plate supplemented with appropriate antibiotic.

LB medium: $25 \mathrm{~g}$ of Luria Broth (Invitrogen) powder was dissolved in 1L of ultrapure water (with conductivity of $18.2 \mathrm{M} \Omega . \mathrm{cm}$ at $25^{\circ} \mathrm{C}$ ) and autoclaved.

LB plate: $25 \mathrm{~g}$ of Luria Broth (Invitrogen) powder and $15 \mathrm{~g}$ of Bacto-agar (Invitrogen) was dissolved in 1L of ultrapure water and autoclaved. After cooling down, stock solution of ampicillin or kanamycin was added to be at the final concentration of $50 \mu \mathrm{g} / \mathrm{mL}$ or $25 \mu \mathrm{g} / \mathrm{mL}$ respectively.

\subsubsection{DNA concentration measurements}

DNA samples were diluted 1:100 by TE buffer and UV absorbance of DNA solution at 260, 280 and $320 \mathrm{~nm}$ wavelength was measured by UltraSpec 3100pro (Amersham). Solutions with 1.8-2.0 $\mathrm{OD}_{260} / \mathrm{OD}_{280}$ ratio were accepted as pure DNA preparations (free from protein and RNA contamination)

\subsubsection{DNA sequencing}

All DNA sequencing was done by the Max Planck Institute of Experimental Medicine DNA Core Facility on an Applied Biosystems 373 DNA Sequencer.

\subsubsection{Polymerase Chain Reaction (PCR)}

DNA fragments of interest were amplified in 25 or $50 \mathrm{uL}$ PCR reaction mixtures containing the double stranded DNA template, oligonucleotide primers, dNTPs, DNA polymerase and appropriate buffer supplied for each DNA polymerase. For cloning, Pfu polymerase (Cloned Pfu Polymerase AD or Turbo Pfu Polymerase AD, Agilent Technologies) was used. For genotyping, Red-Taq DNA polymerase (Sigma-Aldrich) was used. All reactions were run on a Gene Amp 9700 PCR cycler (Applied Biosystems) with the following program;

Step 1: $94^{\circ} \mathrm{C}$ for 3 minuntes,

Step 2: $94^{\circ} \mathrm{C}$ for 30 seconds,

Step 3: annealing temperature for 30 seconds,

Step 4: $72^{\circ} \mathrm{C}$ for an appropriate extension time (30 cycles from Step 2 to 4 ) 
Step 5: $72^{\circ} \mathrm{C}$ for $7 \mathrm{~min}$

Annealing temperature was at least $5^{\circ} \mathrm{C}$ lower than the estimated melting temperature of primers used in reaction. Extension time was set as 1 minute per 250 bp and 500 bp of DNA for Cloned Pfu Polymerase and Turbo Pfu Polymerase respectively.

\subsubsection{TOPO-cloning kit}

In order to subclone the PCR products, pCR2.1-TOPO- or pCRII-TOPO-TA cloning kit (Invitrogen) was used following the manufacturer's protocol.

\subsubsection{DNA digestion with restriction endonucleases}

DNA digestion with specific restriction endonucleases was done using $20 \mathrm{U}$ of a given restriction endonuclease enzyme per $\mu$ g of plasmid DNA for 3 hours with appropriate temperature and buffer condition according to the New England Biolabs (NEB) manual.

\subsubsection{Agarose gel electrophoresis}

For size analyses and purification of DNA, PCR products or digested DNA were subjected to agarose gel electrophoresis in 1-2\% TBE-based agarose gel containing $0.1 \%$ GelRed (Biotium). DNA fragments were separated at the constant voltage of 100V in TBE buffer and then visualized under UV-light system (Intas). Gene Ruler DNA Ladder Mix (Fermantas $100 \mathrm{bp}$ or $1 \mathrm{~kb}$ ) was used to estimate approximate DNA size.

TBE buffer: $50 \mathrm{mM}$ Tris-Base, $50 \mathrm{mM}$ boric acid, $2 \mathrm{mM}$ EDTA, pH 8.0

DNA Loading Dye: $10 \mathrm{mM}$ Tris-HCl pH 7.6, 0.03\% (w/v) bromophenol blue (Sigma-Aldrich), $0.03 \%(\mathrm{w} / \mathrm{v})$ xylene cyanol FF (Sigma-Aldrich), 60\% (v/v) glycerol (Merck), 60 mM EDTA.

\subsubsection{DNA extraction and purification from agarose gel}

After agarose gel electrophoresis, DNA band of interest was excised using a surgical scalpel and DNA fragment was purified from gel piece using PureLink Gel Extraction Kit (Invitrogen) following manufacturer's protocol.

\subsubsection{De-phosphorylation of 5'-DNA ends}

The 5'-ends of DNA fragments with compatible ends were subjected to dephosphorylation in order to prevent the self-ligation of vectors in further ligation procedures. Briefly, plasmid DNA was treated with $2 \mu \mathrm{L}$ of shrimp alkaline phosphatase (Roche) in the 
supplied buffer for $1 \mathrm{~h}$ at $37^{\circ} \mathrm{C}$ in $50 \mu \mathrm{L}$ final volume of reaction. Alkaline phosphatase was inactivated upon incubation at $65^{\circ} \mathrm{C}$ for 20 minutes for further steps of cloning.

\subsubsection{DNA ligation}

Digested vector DNA and digested insert DNA with compatible end were mixed in a molar ratio of 1:1 to $1: 10$, each reaction supplemented with $2 \mathrm{mM}$ ATP, T4 DNA Ligase (Invitrogen), and the ligation buffer in $20 \mu \mathrm{L}$ of reaction mixture. The ligation reaction was performed in ice bucket/water, which provides little temperature increments from $4^{\circ} \mathrm{C}$ to room temperature for 20-24 hours.

\subsubsection{Cloning strategies for construct generated in this study}

pCRII-TOPO-Kir4.1 Cyt

cDNA fragment encoding cytoplasmic C-terminal region of mouse Kir4.1 was amplified by PCR from the pEGFP-C2-Kir4.1 vector using primers 30660/30661 and subcloned into pCRIITOPO vector

\section{pCMV2b-FLAG-Kir4.1 Cyt}

The cytoplasmic $\mathrm{C}$ terminal region of Kir4.1 cDNA was excised from the pCRII-TOPO-Kir4.1 Cyt vector using EcoRI/XhoI and ligated to the EcoRI/XhoI sites of pCMV2bFLAG vector

\section{pCRII-TOPO-Prr7 Cyt}

cDNA fragment encoding the cytoplasmic C-terminal region of mouse Prr7 was amplified by PCR from the pCMV2b-FLAG-Prr7 vector using primers 30925/30954 and subcloned into pCRII-TOPO vector

\section{pRaichuMyc-Prr7 Cyt}

The cytoplasmic region of mouse Prr7 was excised from the pCRII-TOPO-Prr7 Cyt vector using EcoRI/SalI and ligated to the EcoRI/SalI sites of pRaichuMyc

\subsection{Cell Biology}

\subsubsection{Chemical and reagents}

\begin{tabular}{|l|l|}
\hline Product & Company \\
\hline Bovine Serum Albumin & Sigma-Aldrich \\
\hline B27 Serum-Free Supplement (50X) & Thermo-Fischer \\
\hline Calcium Chloride dihydrate & Sigma-Aldrich \\
\hline Dimethyl sulfoxide (DMSO) & Sigma-Aldrich \\
\hline
\end{tabular}




\begin{tabular}{|l|l|} 
Dulbecco's Modified Eagle Medium (DMEM) & Gibco, Invitrogen \\
\hline Dulbecco's Phosphate Buffered Saline (DPBS) & Gibco, Invitrogen \\
\hline EDTA & Sigma-Aldrich \\
\hline Fetal Bovine Serum (FBS) & Gibco, Invitrogen \\
\hline Glutamax (200mM) & Gibco, Invitrogen \\
\hline Glucose & Sigma-Aldrich \\
\hline Hank's Balanced Salt Solution $\left(-\mathrm{Ca}^{2+} / \mathrm{Mg}^{2+}\right)$ & Gibco, Invitrogen \\
\hline HEPES & Sigma-Aldrich \\
\hline L-Cysteine & Sigma-Aldrich \\
\hline Mito+TM Serum Extender & Corning \\
\hline Neurobasal-A Medium & Gibco, Invitrogen \\
\hline Opti-MEM Reduced Serum Medium & Gibco, Invitrogen \\
\hline Papain & Worthington \\
\hline Penicillin/Streptomycin (100X) & Gibco, Invitrogen \\
\hline poly-L Lysine & Sigma-Aldrich \\
\hline Potassium Chloride & Sigma-Aldrich \\
\hline Sodium Butyrare & Merck \\
\hline Sodium Chloride & Sigma-Aldrich \\
\hline Sodium Phosphate Dibasic & Sigma-Aldrich \\
\hline Trypsin-EDTA (0.05\%), phenol red & Gibco, Invitrogen \\
\hline Trypsin Inhibitor & Sigma-Aldrich \\
\hline
\end{tabular}

\subsubsection{Culture media and solutions}

Papain Stock Solution: $0.2 \mathrm{mg} / \mathrm{mL}$ cycteine, $1 \mathrm{mM} \mathrm{CaCl}$, $0.5 \mathrm{mM}$ EDTA in Dulbecco's Modified Eagle's Medium (DMEM)

Papain Working Solution: 20 units of papain (Worthington) were added in $1 \mathrm{~mL}$ of Papain Stock Solution. Solution was saturated with carbogen (95\% oxygen, 5\% carbondioxide) until Papain was resolved, and then it was sterilized using a $0.22 \mu \mathrm{m}$ filter (Millipore).

Stop Solution: $2.5 \mathrm{mg} / \mathrm{mL}$ Bovine Serum Albumin (BSA), $2.5 \mathrm{mg} / \mathrm{mL}$ trypsin inhibitor and 10\% Fetal Bovine Serum (FBS) in DMEM.

Complete Neurobasal Medium: $500 \mathrm{~mL}$ Neurobasal A, $5 \mathrm{~mL}$ GlutaMAX, $10 \mathrm{~mL}$ B-27 supplement (Invitrogen), $1 \mathrm{~mL}$ Penicillin/Streptomycin.

Mito Solution: One vial of Mito ${ }^{+\mathrm{TM}}$ Serum Extender was dissolved in $5 \mathrm{~mL}$ DMEM. It was sterilized using a $0.22 \mu \mathrm{m}$ filter (Millipore), aliquoted and stored at $-20^{\circ} \mathrm{C}$ freezer. 
FBS Astrocyte Medium: $450 \mathrm{~mL}$ DMEM, $50 \mathrm{mM}$ FBS, $5 \mathrm{~mL}$ GlutaMAX, $1 \mathrm{~mL}$ Penicillin/Streptomycin, $500 \mu \mathrm{L}$ Mito Solution.

10\% FBS/DMEM: $450 \mathrm{~mL}$ DMEM, $50 \mathrm{~mL}$ FBS, $5 \mathrm{~mL}$ GlutaMAX, $5 \mathrm{~mL}$ Penicillin/Streptomycin.

10\% FBS/OPTI-MEM: 450 mL OPTI-MEM, 50 mL FBS.

2\% FBS/Sodium Butyrate/DMEM: $1 \mathrm{~mL}$ of FBS in $50 \mathrm{~mL}$ DMEM, $10 \mathrm{mM}$ Sodium Butyrate.

\subsubsection{HEK293FT cell line}

HEK293FT cells (Invitrogen) were maintained in 10\% FBS/DMEM using culture petri dishes (Corning) in $37^{\circ} \mathrm{C}$ incubator with $5 \% \mathrm{CO}_{2}$ (HERA-Cell 240 Heraeus). For passaging, confluent cells were washed with PBS followed by incubation with $1 \mathrm{~mL} 0.05 \%$ trypsin-EDTA solution for $3 \mathrm{~min}$ at $37^{\circ} \mathrm{C}$. Reaction was stopped by addition of $9 \mathrm{~mL}$ of $10 \% \mathrm{FBS} / \mathrm{DMEM}$. Cells were then split on new petri dishes.

\subsubsection{Freezing HEK293FT cell line}

Confluent HEK293FT cells in $15 \mathrm{~cm}$ dish (Corning) were washed with PBS and treated with $0.05 \%$ Trypsin-EDTA Solution for $3 \mathrm{~min}$ at $37^{\circ} \mathrm{C}$. Cells were harvested in $10 \mathrm{~mL}$ of $10 \%$ FBS/DMEM and centrifuged at $800 \mathrm{~g}$ for $5 \mathrm{~min}$. Later, cell pellet was resuspended in $1 \mathrm{~mL}$ of 10\% FBS/DMEM and $1 \mathrm{~mL}$ of Freezing Medium per each dish was added dropwise to the cell suspension. Next, $1 \mathrm{~mL}$ of cells in freezing medium was aliquoted into cryo-tubes and frozen at $-80^{\circ} \mathrm{C}$ in Cryo Freezing Container (Nalgene) filled with isopropanol. One aliquot of cell suspension was thawn into new $15 \mathrm{~cm}$ dish to achieve approximately $50 \%$ confluency for the first day of seeding.

2X Freezing Medium: 20\% DMSO, 20\% FBS in DMEM

\subsubsection{Transfection of HEK293FT cells}

Transfection of HEK293FT was carried out at 80-90\% cell confluency using Lipofectamine2000 (Invitrogen) following manufacturer's instructions.

\subsubsection{Primary mouse hippocampal culture preparation}


Hippocampi of P0 mice were dissected in ice-cold HBSS under stereomicroscope and incubated in $1 \mathrm{~mL}$ of Papain Working Solution at $37^{\circ} \mathrm{C}$ with gentle shaking for $1 \mathrm{~h}$. Papain solution was replaced by Stop Solution pre-warmed at $37^{\circ} \mathrm{C}$. After incubation of hippocampi at $37^{\circ} \mathrm{C}$ for 15 minutes with gentle agitation, Stop Solution was discarded and the hippocampi were washed twice with $500 \mu \mathrm{L}$ of Complete Neurobasal Medium pre-warmed at $37^{\circ} \mathrm{C}$. Next, medium was removed and hippocampi were triturated with $200 \mu \mathrm{L}$ Complete Neurobasal Medium 10 times using P200 pipette tip. The sample was left in the cell culture hood for 1 minute to let debris to sink, isolated neurons in the supernatant was harvested and transferred into $1 \mathrm{~mL}$ of Complete Neurobasal Medium pre-warmed at $37^{\circ} \mathrm{C}$. The trituration was repeated using $200 \mu \mathrm{L}$ fresh Complete Neurobasal Medium and supernatant was collected in same eppendorf tube. Cells were counted using Naubauer counting chamber and neurons were seeded on coverslips or on plastic wells coated by poly-L-lysine (Sigma-Aldrich) for immunocytochemistry or for biochemistry respectively. For immunocytochemistry experiments, 40,000-70,0000 cells were plated per each well of 24-well plate. For biochemical experiments, 120,000 or 500,000 cells were seeded per each well of 24- or 6-well plate respectively. The day of culture preparation was counted as day in vitro 0 (DIV0). Neurons were maintained in $1 \mathrm{~mL}$ or $2 \mathrm{~mL}$ of Complete Neurobasal Medium per each well of 24 - or 6-well plate at $37^{\circ} \mathrm{C}$ with $5 \% \mathrm{CO}_{2}(\mathrm{HERA}-\mathrm{cell} 240$ incubator, Heraeus). After 2-4 hours of seeding the cells, the medium was changed to fresh Complete Neurobasal Medium.

\subsubsection{Transfection of primary hippocampal culture}

Primary cultured hippocampal neurons were transfected at DIV1-3 using calcium phosphate transfection method. Briefly, Complete Neurobasal Medium in each well was replaced with $500 \mu \mathrm{L}$ of plane pre-warmed Neurobasal Medium and kept at $37^{\circ} \mathrm{C}$. For one well of 24-well plate, $1 \mu \mathrm{g}$ of plasmid DNA was mixed with $3.1 \mu \mathrm{L}$ of $2 \mathrm{M} \mathrm{CaCl}_{2}$ and the volume of the mixture was adjusted to $25 \mu \mathrm{L}$ with sterile ultra-pure water. The DNA mixture was added to $25 \mu \mathrm{L}$ of $2 \mathrm{XHBS}$ dropwise with a gentle agitation. After incubation of the transfection solution for 30 minutes in the dark, $50 \mu \mathrm{L}$ of the transfection solution was applied on each well. The plate was incubated in $37^{\circ} \mathrm{C}$ incubator with $5 \% \mathrm{CO}_{2}$ for 20 to 25 minutes until the precipitates were observed under the light microscope. Each well was subsequently washed three times with $500 \mu \mathrm{L}$ of $\mathrm{HBSS}$ pre-equilibrated at $37^{\circ} \mathrm{C}$ in $10 \% \mathrm{CO}_{2}$ until calcium phosphate-DNA precipitates 
were resolved. Original Complete Neurobasal Medium was placed on the wells and plates were maintained at $37^{\circ} \mathrm{C}$ incubator with $5 \% \mathrm{CO}_{2}$.

2X HBS: 274mM NaCl, 10 mM KCl, 1.4 mM Na2HPO4, 15 mM Glucose, 42 mM HEPES, pH7.8 adjusted with $10 \mathrm{M} \mathrm{NaOH}$.

\subsubsection{Primary mouse cortical culture preparation}

Cortices from one E16 embryo were dissected on ice-cold Hank's Balanced Salt Solution (HBSS, Gibco, Life Sciences) under stereomicroscope and incubated in $1 \mathrm{~mL}$ of Papain Working Solution at $37^{\circ} \mathrm{C}$ with gentle agitation for 1 hour. Papain digestion and the trituration of the tissue, and plating of the neuron were performed in the same way as for hippocampal neurons.

\subsubsection{Primary mouse astrocyte culture preparation}

Cortices from one P0 mouse were dissected on ice-cold Hank's Balanced Salt Solution (HBSS) under stereomicroscope and incubated in $1 \mathrm{~mL}$ of trypsin solution at $37^{\circ} \mathrm{C}$ with gentle agitation for 15 minutes. Then, trypsin solution was removed and cortices were wash three times with $1 \mathrm{~mL}$ of FBS Astrocyte Medium pre-incubated in $37^{\circ} \mathrm{C}$ incubator with $5 \% \mathrm{CO}_{2}$. After the final wash, cortices were triturated 20 times with $750 \mu \mathrm{L}$ of fresh FBS Astrocyte Medium using P1000 pipette tip. After incubation in the cell cultre hood for 1 minute, dissociated astrocytes in the supernatant was transferred into $10 \mathrm{~mL}$ of FBS Astrocyte Medium in a $75 \mathrm{~cm}$ flask (Corning). Next day, the medium was changed to fresh FBS Astrocyte Medium to remove the floating cell clamps. Cultures were maintained until they became confluent at $37^{\circ} \mathrm{C}$ in $5 \% \mathrm{CO}_{2}$. Then, cells were split using trypsin and seeded on coverslips or into 6-well plates coated with poly-L-lysine for calcium imaging and for biochemistry experiments respectively.

\subsubsection{Lentivirus preparation}

HEK293FT cells were used to produce lentivirus particles. Briefly, HEK293FT cells were cultured in 10\% FBS/DMEM in $15 \mathrm{~cm}$ petri dish until confluency and one plate was split into two poly-L-lysine coated $15 \mathrm{~cm}$ dish so that the confluency will be $80-90 \%$ for optimal transfection in 24 hours. Next day, 10\% FBS/DMEM was placed with $20 \mathrm{~mL}$ of $10 \%$ FBS/OPTIMEM pre-warmed at $37^{\circ} \mathrm{C}$ water bath. The cells were co-transfected with $40 \mu \mathrm{g}$ of lentivirus backbone vector (FUGW or FUGW-iCre) with $16 \mu \mathrm{g}$ of plasmids encoding Gag polyprotein 
(pCMVdeltaR8.2) and envelope protein (pMD2.G) using Lipofectamine2000. Cultures were maintained in $37^{\circ} \mathrm{C}$ incubator with $5 \% \mathrm{CO}_{2}$. After 8 hours of transfection, $10 \%$ FBS/OPTI-MEM was changed to $20 \mathrm{~mL}$ of $2 \% \mathrm{FBS} /$ Sodium Butyrate/DMEM pre-warmed to $37^{\circ} \mathrm{C}$ and plates were incubated at $37^{\circ} \mathrm{C}$ incubator with $5 \% \mathrm{CO}_{2}$. At 48 hours post-transfection, culture medium was harvested in $50 \mathrm{~mL}$ Falcon tubes and cell debris was spin down at $2000 \mathrm{~g}$ at $4^{\circ} \mathrm{C}$ for $10 \mathrm{~min}$. Supernatant was filtered using $45 \mu \mathrm{m}$ filters (Millipore) and applied to AMICON Centrifugal Filter (100 kDa, Millipore) to concentrate viral particles by centrifugation at $3500 \mathrm{~g}$ at $4^{\circ} \mathrm{C}$. The volume of concentrated virus solution was adjusted to $1 \mathrm{~mL}$ per $15 \mathrm{~cm}$ petri dish with cold Neurobasal-A Medium and solution was dialyzed overnight against TBS at $4^{\circ} \mathrm{C}$ in a sterilized beaker using Slide-A-Lyzer ${ }^{\mathrm{TM}}$ dialysis casette (Thermo-Scientific). After dialysis, virus solution was flash-frozen as aliquots in liquid nitrogen and stored at $-80{ }^{\circ} \mathrm{C}$ until used.

TBS: 20mM Tris-Cl pH 8.0, $150 \mathrm{mM} \mathrm{NaCl}$

\subsubsection{Lentiviral infection of neurons}

After lentivirus preparation, virus solution was applied to cultured neurons at DIV1. After 48 hours of infection, cultures were fixed, stained for GFP, MAP2 and DAPI and observed under fluorescent microscope. Appropriate amount of virus solution was determined as the amount of virus solution resulting expression of GFP in all DAPI or MAP2 positive cells. For experiments, determined virus amounts was applied to each well at DIV1 and cells were fixed for immunocytochemistry or harvested for protein chemistry after washing four times with PBS at appropriate stages of the culture.

\subsubsection{Immunocytochemistry}

Neurons cultured on coated coverslips were fixed with Fixation Buffer for 15 min in cold room under gentle agitation. After washing three times with PBS, cells were incubated with Blocking Buffer at room temperature for 1 hour. Fixed cells were incubated with primary antibodies diluted in Blocking Buffer (dilutions in Table 7) for 20-24 hours at $4^{\circ} \mathrm{C}$ in humidified chamber with gentle agitation. After incubation with the primary antibody, cells were washed three times for 15 minutes with PBS at room temperature on moderate shaking. Next, cells were incubated with secondary antibodies diluted in Blocking Buffer for 1 hour at room temperature. After washing the cells with PBS three times for 15 minutes and once brief rinsing with ultra- 
pure water, coverslips were mounted on glass slides (Superfrost Plus, Thermo Scientific) with Immu-Mount mounting medium (Shandon, Thermo-Scientific).

Fixation Buffer: $4 \%$ paraformaldehyde (PFA), 4\% Sucrose in PB

Blocking Buffer: $5 \%$ Goat serum, $0.3 \%$ Triton X-100, 0.1\% fish skin gelatin in PBS

\subsubsection{Sholl analysis}

In order to analyze the dendrite arborization, EGFP-expressing primary hippocampal neurons were fixed at DIV7 and images were acquired using $25 \mathrm{X}$ objective (with water immersion) of AxioImager Z.1 (Carl Zeiss). After thresholding and binarization of images in ImageJ, Sholl analysis was applied with $10 \mu \mathrm{m}$ interval between Sholl circles. Total numbers of crossings on Sholl circles were quantified in given distances for each neuron. For statistical analysis, t-test was used to compare two independent samples after f-test to detect t-test type.

\subsection{Biochemistry}

\subsubsection{Mouse brain homogenization}

Mice were anesthetized and decapitated. The cortices were dissected in cold $0.32 \mathrm{M}$ Sucrose and homogenized with 10 strokes at $900 \mathrm{rpm}$ (Braun POTTER'S Homogenizer) in 2 $\mathrm{mL}$ of Homogenization solution in a Teflon-glass homogenizer (Sartorius) at $4^{\circ} \mathrm{C}$.

Homogenization solution: $0.32 \mathrm{M}$ Sucrose, $0.1 \mu \mathrm{M}$ Aprotinin, $50 \mu \mathrm{M}$ Leupeptin, $0.2 \mathrm{mM}$ PMSF

\subsubsection{Harvesting cell cultures}

For biochemical experiments, neurons at appropriate stage of cell culture were washed three times with PBS and harvested with $50 \mu \mathrm{L}$ or $150 \mu \mathrm{L}$ of Laemmli Buffer for each well of 24-well plate or 6-well plate respectively.

Laemmli Buffer: 10\% Glycerol, 50 mM Tris-Cl pH 6.8, 2 mM EDTA, 2\% SDS, 100 mM DTT, $0.05 \%$ Bromophenol blue

\subsubsection{Measurement of protein concentration}

Protein amount in samples were measured using Bradford (Bio-Rad) or the bichichoninic acid (BCA, Thermo Scientific) kit according to the manufacturer's instructions. 
Briefly, protein samples were incubated with the Bradford solution for 1 min at room temperature or with the $\mathrm{BCA}$ reagent at $37^{\circ} \mathrm{C}$ for $30 \mathrm{~min}$. Next, the abrorbance at $560 \mathrm{~nm}$ or 652 $\mathrm{nm}$ of each samples was measured for Bradford or BCA kit respectively. The BSA samples with known concentrations were used as standards in order to estimate protein concentrations of individual samples.

\subsubsection{Purification of synaptic plasma membrane fraction}

Cortices of 6 weeks old mice were dissected in ice cold Solution A using a petri dish on ice. After homogenization in $2 \mathrm{~mL}$ Solution A with 10 strokes at $900 \mathrm{rpm}$, homogenates were applied to discontinuous sucrose density gradient with $0.85 \mathrm{M}, 1.0 \mathrm{M}$ and $1.2 \mathrm{M}$ sucrose layers. After centrifugation at 82,500 g for 2 hours at $4{ }^{\circ} \mathrm{C}$ (Rotor SW41, Beckmann Ultracentrifuge), the supernatant above the $0.85 \mathrm{M}$ sucrose were collected as soluble fraction $(\mathrm{S})$ and interfaces between $0.32 \mathrm{M}-0.85 \mathrm{M}$, between $0.85-1.0 \mathrm{M}$, and between $1.0-1.2 \mathrm{M}$ were harvested as myeline-enriched fraction (P2A), ER-Golgi-Enriched Fraction (P2B), and synaptosome fraction (P2C). The pellet was collected as mitochondria-enriched fraction (P2D). The P2C fraction then diluted 1:2 with Solution A and centrifuged at $100000 \mathrm{~g}$ for 20 minutes (TLA100.3 Rotor). The pellet of P2C was resuspended in $2.5 \mathrm{~mL}$ of $6 \mathrm{mM}$ Tris-Cl, $\mathrm{pH} 8$ using $21 \mathrm{G}$ and $27 \mathrm{G}$ needles (BD Microlance) and incubated on ice for 45 minutes for osmotic shock. Then, samples were centrifuged at $32.8 \mathrm{~kg}$ for 20 minutes (TLA100.3 Rotor) in order to separate synaptic membranes from synaptosomes. The supernatant was harvested as synaptic cytoplasm (SC) and crude synaptic vesicle (CSV) fraction while pellet was resuspended as crude synaptic membrane (CSM) fraction with $3 \mathrm{~mL}$ of Solution A using $21 \mathrm{G}$ and $27 \mathrm{G}$ neddles. Further, the CSM fraction was applied to discontinuous sucrose density gradient with $0.85 \mathrm{M}, 1.0 \mathrm{M}, 1.2 \mathrm{M}$ sucrose layers followed by centrifugation at $82500 \mathrm{~g}$ for 2 hours at $4{ }^{\circ} \mathrm{C}$ (Rotor SW41, Beckmann Ultracentrifuge). The interface between 1.0 M-1.2 M sucrose was harvested as pure synaptic membrane fraction (SM3) and diluted 1:2 with solution A followed by centrifugation at 100000 $\mathrm{g}$ for 20 minutes at $4^{\circ} \mathrm{C}$ (TLA100.3). Later, the pellet was resuspended in $500 \mu \mathrm{L}$ of $6 \mathrm{mM}$ Tris$\mathrm{Cl}$ pH 8.0 using $21 \mathrm{G}$ and $27 \mathrm{G}$ needles. Purified fractions were stored in $-80^{\circ} \mathrm{C}$ freezer and used for mass-spectroscopy analysis, western blots for verification of mass-spectroscopy results or protein localization experiment.

Solution A: $0.32 \mathrm{M}$ Sucrose, $1 \mathrm{mM} \mathrm{NaHCO}, 2 \mathrm{mM}$ PMSF, $1 \mu \mathrm{g} / \mathrm{mL}$ Aprotinin, $0.5 \mu \mathrm{g} / \mathrm{mL}$ Leupeptin 


\subsubsection{Sodium dodecyl sulfate polyacrylamide gel electrophoresis (SDS-PAGE)}

Protein samples were applied to SDS-PAGE under the denaturating conditions in order to separate proteins based on their molecular sizes. Briefly, protein samples prepared in Laemmli buffer was boiled at $65^{\circ} \mathrm{C}$ for 20 minutes and loaded onto two-layered polyacrylamide gel consisting the upper stacking gel and lower separating gel (Bio-Rad Mini-PROTEAN 251 Casting System). Electrophoresis was performed with a constant current at 20-30 mA per gel in Bio-Rad electrophoresis system filled with Running Buffer. The PageRuler or PageRuler Plus Prestained Protein Ladders (Thermo Scientific) were used as protein markers.

Laemmli Buffer: 10\% Glycerol, 50 mM Tris-Cl pH 6.8, 2 mM EDTA, 2\% SDS, 100 mM DTT, 0.05\% Bromophenol blue

Upper Stacking Gel: 5\% AMBA, $125 \mathrm{mM}$ Tris-Cl pH 6.8 at RT, 0.1\% SDS, 0.05\% APS, 0.005\% TEMED

Lower Seperating Gel: 7-15\% AMBA, $325 \mathrm{mM}$ Tris-Cl pH 8.8 at RT, $0.1 \%$ SDS, $0.05 \%$ APS, $0.005 \%$ TEMED

Running Buffer: $25 \mathrm{mM}$ Tris-Cl pH 8.8 at RT, $250 \mathrm{mM}$ Glycine, $0.1 \%$ SDS

\subsubsection{Western blotting}

Proteins separated in SDS-PAGE were transferred onto a nitrocellulose (Protran $0.2 \mu \mathrm{m}$, GE Healthcare) or PVDF membrane (Hybond $0.2 \mu \mathrm{m}$, GE Healthcare) with a constant current of $80 \mathrm{~mA}$ or $200 \mathrm{~mA}$ for 10 hours respectively. Proteins were transferred in a blotting tank unit (Hoefer, TE22) filled with Transfer Buffer. After transfer, membranes were washed with ultrapure water and incubated with MemCode Reversible Protein Stain Kit in order to stain proteins transferred on nitrocellulose membranes (Thermo Scientific) according to manufacturer's protocol. After destaining and washing, membranes were incubated in Blocking Buffer for 1 hour at room temperature with moderate shaking followed by incubation with primary antibodies listed in Table 7 for 3 hours at room temperature with gentle agitation. Membranes were washed three times with Washing Buffer for 15 minutes and incubated with secondary antibodies listed in Table 8 for 1 hour at room temperature. Secondary antibodies nonspecifically attached on membranes were washed with Washing Buffer three times for 15 minutes. The signal on the membrane was detected with enhanced chemiluminescence (ECL) system (Solution I and II, GE Healthcare) or with Odyssey Infrared Imaging System (LI-COR 
Biosciences). Quantification of protein bands was performed using the Image-Studio Software (Odyssey System) or ImageJ.

Transfer Buffer: $25 \mathrm{mM}$ Tris-base, $190 \mathrm{mM}$ Glycine. 20\% methanol

TBS: $20 \mathrm{mM}$ Tris-Cl pH 7.5 at RT, $137 \mathrm{mM} \mathrm{NaCl}$

Blocking Buffer: $5 \%$ milk powder in TBS-T

Washing Buffer: $1 \%$ Tween20 (w/v) (Sigma-Aldrich) in TBS

\subsection{7 iTRAQ labelling and quantitative mass spectroscopy}

iTRAQ labelling of SM3 fractions was performed by Dr. Olaf Jahn using an automated in-gel digestion/iTRAQ-labelling method (Schmidt et al., 2013). Mass spectrometry was performed in collaboration with Dr. Samir Karaca and Dr. Henning Urlaub.

\subsubsection{Purification of recombinant mouse ubiquitin activating enzyme (E1)}

For in vitro ubiquitination assays, recombinant mouse ubiquitin activating enzyme E1 was expressed in E. coli and purified according to published protocols (Carvalho et al., 2012) with slight modifications. Briefly, pET28-mE1 plasmid was electro-transformed into BL21(DE3) E. coli cells and plated on LB-agar plate supplemented with kanamycin. After overnight incubation, colony was picked and inoculated into $50 \mathrm{~mL}$ LB medium supplemented with $25 \mu \mathrm{g} / \mathrm{mL}$ kanamycin and grown overnight at $37^{\circ} \mathrm{C}$. The pre-culture was diluted to 1 liter of fresh LB medium with kanamycin and grown at $37^{\circ} \mathrm{C}$ until the optical density at $600 \mathrm{~nm}$ $\left(\mathrm{OD}_{600}\right)$ reached 0.6. Next, the culture was cooled down to $16^{\circ} \mathrm{C}$ and recombinant protein expression was induced by the addition of $0.5 \mathrm{mM}$ isopropyl- $\beta$-D-1-thiogalactosypyranoside (IPTG) for 20 hours at $16^{\circ} \mathrm{C}$. Later, the $E$. coli cells were pelleted by centrifugation at $3500 \mathrm{~g}$ at $4^{\circ} \mathrm{C}$ for 45 minutes and resuspended in $25 \mathrm{~mL}$ of ice-cold Lysis Buffer A followed by flashfreezing in liquid nitrogen and storage at $-80^{\circ} \mathrm{C}$ for at least one overnight. Further, lysate was thawn on ice, and sonicated with 30\% of maximum power using Sonopuls HD 2200 Sonicator equipped with Probe VS 70 two times for 20 seconds in ice-cold water with 20 second intervals of incubation on ice. After sonication, proteins were solubilized by addition of the same volume of Lysis Buffer B. Insoluble fractions were removed by centrifugation at $10000 \mathrm{~g}$ for $10 \mathrm{~min}$ at $4^{\circ} \mathrm{C}$ (Rotor 22.5, Heraeus Varifuge 20 RS Centrifuge) and supernatant was incubated with 1.5 $\mathrm{mL}$ bed volume of His-nickel beads for 6 hours at $4^{\circ} \mathrm{C}$ with gentle rotation. Later, beads were spin downed by centrifugation at $800 \mathrm{~g}$ for 1 minute and washed three times with $30 \mathrm{~mL}$ of 
Wash Buffer A and two times with $30 \mathrm{~mL}$ of Wash Buffer B. The recombinant proteins were eluted from beads three times with $2.5 \mathrm{~mL}$ of Elution Buffer. The eluted proteins were concentrated and buffer-exchanged to Exchange Buffer using Vivaspin 10000 MWCO concentrator (Sartorius). Purified His6-fused mouse E1 enzymes were aliquoted, flash frozen in liquid nitrogen and stored $-80{ }^{\circ} \mathrm{C}$ until use.

Lysis Buffer A: 50 mM Tris-Cl pH 8.0, 150 mM NaCl, 1 mM EDTA-NaOH pH8, 1 mM DTT, $0.2 \mathrm{mM}$ PMSD, $1 \mu \mathrm{g} / \mathrm{mL}$ Aprotinin, $0.5 \mu \mathrm{g} / \mathrm{mL}$ Leupeptin

Lysis Buffer B: 50 mM Tris-Cl pH 8.0, $150 \mathrm{mM} \mathrm{NaCl}, 0.2 \%$ (w/v) Triton X-100, 1 mM EDTA$\mathrm{NaOH}$ pH8, 1 mM DTT, $0.2 \mathrm{mM}$ PMSD, $1 \mu \mathrm{g} / \mathrm{mL}$ Aprotinin, $0.5 \mu \mathrm{g} / \mathrm{mL}$ Leupeptin Wash Buffer A: $50 \mathrm{mM}$ Sodium Phosphate $\mathrm{pH} 8.0,300 \mathrm{mM} \mathrm{NaCl}, 20 \mathrm{mM}$ Imidazole Wash Buffer B: $50 \mathrm{mM}$ Sodium Phosphate $\mathrm{pH}$ 8.0, $150 \mathrm{mM} \mathrm{NaCl}, 20 \mathrm{mM}$ Imidazole Elution Buffer: $50 \mathrm{mM}$ Sodium Phosphate $\mathrm{pH} 8.0,150 \mathrm{mM} \mathrm{NaCl}, 100 \mathrm{mM}$ Imidazole Exchange Buffer: $10 \mathrm{mM}$ Tris-Cl pH 8.0, 1 mM EDTA-NaOH pH 8.0, 1 mM DTT

\subsubsection{Purification of recombinant ubiquitin conjugating enzymes (E2s)}

pGEX-4T1-UbcH5b, -UbcH6, -UbcH7 and pGEX-4P-UbcH5c were electrotransformed into BL21(DE3) E. coli cells and plated on LB-agar plate supplemented with ampicillin. After overnight incubation, colony was picked and inoculated into $50 \mathrm{~mL}$ LB medium supplemented with $25 \mu \mathrm{g} / \mathrm{mL}$ ampicillin and grown overnight at $37^{\circ} \mathrm{C}$. The pre-culture was diluted to 1 liter of fresh $\mathrm{LB}$ medium with ampicillin and grown at $37^{\circ} \mathrm{C}$ until the optical density at $600 \mathrm{~nm}\left(\mathrm{OD}_{600}\right)$ reached 0.5-0.6. Next, the culture was cooled down to $20^{\circ} \mathrm{C}$ and recombinant protein expression was induced by the addition of $1 \mathrm{mM} \mathrm{IPTG}$ for 6 hours at $20^{\circ} \mathrm{C}$. After harvesting by centrifugation at $3500 \mathrm{~g}$ at $4^{\circ} \mathrm{C}$ for 45 minutes, E. coli pellet was resuspended in $30 \mathrm{~mL}$ of ice-cold Lysis Buffer, flash-frozen in liquid nitrogen and stored at $-80^{\circ} \mathrm{C}$ for at least one overnight. Later, lysate was thawn on ice, and supplemented with $1 \mathrm{mg} / \mathrm{mL}$ Lysozyme (Roth) and incubated for 1 hour on ice. Insoluble material was removed by centrifugation at 10 $000 \mathrm{~g}$ for 10 minutes at $4^{\circ} \mathrm{C}$. The supernatant was incubated for 2 hours at $4^{\circ} \mathrm{C}$ on gentle rotation with $1.5 \mathrm{~mL}$ bed volume of Glutathione Sepharose 4B beads (GST-beads, GE-Healthcare) preequilibrated with ice-cold Lysis Buffer. The GST-beads were harvested by centrifugation at 800 $\mathrm{g}$ for $1 \mathrm{~min}$ at $4^{\circ} \mathrm{C}$ and washed 4 times with ice-cold $30 \mathrm{~mL}$ of Washing Buffer and 2 times with $30 \mathrm{~mL}$ of Cleavage Buffer. The beads were harvested in $5 \mathrm{~mL}$ of Cleavage Buffer and incubated with $5 \mu \mathrm{L}$ of untagged thrombin (restriction grade, Millipore). 
Lysis Buffer: $50 \mathrm{mM}$ Tris-Cl pH 8.0, $300 \mathrm{mM} \mathrm{NaCl}, 1 \mathrm{mM}$ DTT, $0.1 \mathrm{mM}$ PMSF, $1 \mu \mathrm{g} / \mathrm{mL}$ Aprotinin, $1 \mu \mathrm{g} / \mathrm{mL}$ Leupeptin

Washing Buffer: $50 \mathrm{mM}$ Tris-Cl pH 8.0, $500 \mathrm{mM} \mathrm{NaCl}, 1 \mathrm{mM}$ DTT, $0.1 \mathrm{mM}$ PMSF, $1 \mu \mathrm{g} / \mathrm{mL}$ Aprotinin, $1 \mu \mathrm{g} / \mathrm{mL}$ Leupeptin

Cleavage Buffer: $50 \mathrm{mM}$ Tris-Cl pH 8.0, $100 \mathrm{mM} \mathrm{NaCl}, 1 \mathrm{mM}$ DTT, $0.1 \mathrm{mM}$ PMSF, $1 \mu \mathrm{g} / \mathrm{mL}$ Aprotinin, $1 \mu \mathrm{g} / \mathrm{mL}$ Leupeptin

\subsubsection{Purification of recombinant ubiquitin ligase enzymes (E3s)}

pGEX4T-WWP1, pGEX4T-Itch, pGEX6P-WWP2, pDEST-Nedd4-1 or pDESTNedd4-2 were electro-transformed into BL21 (DE3) E. coli cells and plated on LB agar with ampicillin. After overnight incubation, colony was picked and inoculated into $50 \mathrm{~mL} \mathrm{LB}$ medium supplemented with $25 \mu \mathrm{g} / \mathrm{mL}$ ampicillin and grown overnight at $37^{\circ} \mathrm{C}$. The pre-culture was diluted to 1 liter of fresh $\mathrm{LB}$ medium with ampicillin and grown at $16^{\circ} \mathrm{C}$ until the optical density at $600 \mathrm{~nm}\left(\mathrm{OD}_{600}\right)$ reached 0.5-0.6. Next, recombinant protein expression was induced by the addition of $0.5 \mathrm{mM}$ IPTG for 6 hours at $20^{\circ} \mathrm{C}$. After harvesting by centrifugation at 3500 $\mathrm{g}$ at $4^{\circ} \mathrm{C}$ for 45 minutes, E. coli pellet was resuspended in $30 \mathrm{~mL}$ of ice-cold Lysis Buffer, flashfrozen in liquid nitrogen and stored at $-80^{\circ} \mathrm{C}$ for at least one overnight. Later, lysate was thawn on ice, and supplemented with $1 \mathrm{mg} / \mathrm{mL}$ Lysozyme (Roth) and incubated for 1 hour on ice. Insoluble material was removed by centrifugation at $10000 \mathrm{~g}$ for 10 minutes at $4^{\circ} \mathrm{C}$. The supernatant was incubated for 2 hours at $4^{\circ} \mathrm{C}$ on gentle rotation with $1.5 \mathrm{~mL}$ bed volume of Glutathione Sepharose 4B beads (GST-beads, GE-Healthcare) pre-equilibrated with ice-cold Lysis Buffer. The GST-beads were harvested by centrifugation at $800 \mathrm{~g}$ for $1 \mathrm{~min}$ at $4^{\circ} \mathrm{C}$ and washed 4 times with ice-cold $30 \mathrm{~mL}$ of Washing Buffer and 2 times with $30 \mathrm{~mL}$ of Cleavage Buffer. The beads were harvested in $5 \mathrm{~mL}$ of Cleavage Buffer and incubated with $5 \mu \mathrm{L}$ of untagged thrombin (restriction grade, Millipore)

\subsubsection{Immunoprecipitation of proteins from HEK cell lysates}

In order to conduct in vitro ubiquitination assay, tagged (myc or FLAG) substrate proteins were overexpressed in HEK293FT cells for 48 hours after transfection. Later, cells were washed in PBS and harvested in $1 \mathrm{~mL}$ of Lysis Buffer and incubated for 30 minutes on ice. Next, 
lysates were cleared by centrifugation $10000 \mathrm{rpm}$ for 10 minutes at $4^{\circ} \mathrm{C}$ and supernatant was used for immunoprecipitation for tagged proteins.

\subsubsection{In vitro Ubiquitination Assay for Substrates Expressed in HEK293FT cells}

The in vitro ubiquitination reactions were performed in $20 \mu \mathrm{L}$ reactions in order to prove that substrates were ubiquitinated specifically by E3 ligases. Substrates were expressed in HEK293FT cells (grown in $10 \mathrm{~cm}$ petri dish) and immunoprecipitated using $10 \mu \mathrm{L}$ myc-beads (Sigma-Aldrich) or $10 \mu \mathrm{L}$ FLAG-beads (Sigma-Aldrich) per dish. Later, beads were washed three times with $1 \mathrm{~mL}$ of Reaction Buffer and $5 \mu \mathrm{L}$ of beads was used as substrate for each reaction. For each assay, substrate was incubated with $2 \mathrm{mM}$ ATP, $1 \mu \mathrm{M}$ His-Ubiquitin (Boston Biochem), 50 nM mouse E1 enzyme, 150 mM E2 enzyme (UbcH5b, UbcH5c, UbcH6, or UbcH7), 600 nM E3 enzyme purified from E. coli (Nedd4-1, Nedd4-2, WWP1, WWP2 or Itch). Reactions were incubated at $37^{\circ} \mathrm{C}$ for 1 hour with gentle agitation. Later, reactions were stopped by addition of $100 \mu \mathrm{L}$ of $1 \mathrm{X}$ Laemmli Buffer, and results were analyzed by western blotting. Ubiquitin western blotting was used as positive control for each assay, while western blotting for substrate was used to show specific ubiquitination of the substrate only in the presence of E2 and E3 enzymes. Reactions lacking only E2 enzyme, only E3 enzyme, or only substrate were used as negative controls.


Tween

\subsubsection{In vitro Ubiquitination Assay for Substrates Purified from E. coli}

For the ubiquitination assay using VCP as a substrate, recombinant myc-His-VCP purified from $E$. coli were used. Briefly, in the presence of $2 \mathrm{mM}$ ATP and $1 \mu \mathrm{M}$ ubiquitin, 2 $\mu \mathrm{M}$ VCP were incubated with $50 \mathrm{nM}$ mouse E1 enzyme, $150 \mathrm{mM}$ E2 enzyme (UbcH5b, UbcH6 and UbcH7) $600 \mathrm{nM} \mathrm{E3} \mathrm{enzyme} \mathrm{(WWP1,} \mathrm{WWP2,} \mathrm{Itch)} \mathrm{in} 20 \mu \mathrm{L}$ reaction in reaction buffer. After 1 hour incubation at $37^{\circ} \mathrm{C}$, reactions were stopped by addition of $1 \mathrm{X}$ Laemmli Buffer and results were analyzed by western blotting for ubiquitin to show enzymatic activity and for myc to show ubiquitination of VCP by E3 ligases.

Reaction Buffer: $50 \mathrm{mM}$ Tris- $\mathrm{Cl} \mathrm{pH} 8$ at $37^{\circ} \mathrm{C}, 100 \mathrm{mM} \mathrm{NaCl}, 5 \mathrm{mM} \mathrm{MgCl}, 1 \mathrm{mM}$ DTT, $0.05 \%$ Tween 


\subsubsection{In vivo Ubiquitination Assay}

In order to show ubiquitination of substrates in vivo, cell based ubiquitination assay were performed using HEK293FT cells. Briefly, substrate and E3 ligase were co-overexpressed in HEK293FT cells with ubiquitin for 48 hours after transfection. Cells were washed with PBS/NEM and harvested in $200 \mu \mathrm{L}$ of Ubiquitination Buffer containing 1\% SDS and boiled at $65^{\circ} \mathrm{C}$ for 20 minutes. Later, SDS were neutralized by diluting 10 times (up to $2 \mathrm{~mL}$ ) with Ubiquitination Buffer with 1\% Triton X-100. Cell lysate were cleared using centrifugation at 10 $000 \mathrm{rpm}$ for $10 \mathrm{~min}$ at $4^{\circ} \mathrm{C}$. Substrates were then immunoprecipitated using GFP-trap beads (Chromotek), or HA-beads (Sigma-Aldrich), or FLAG-beads (Sigma-Aldrich). After washing three times with Washing Buffer with 1\% Triton X-100, beads were harvested with Laemmli Buffer and subjected to SDS-PAGE and western blotting.

NEM: $125 \mathrm{mg}$ NEM was dissolved in $1 \mathrm{~mL} \mathrm{100 \%} \mathrm{Ethanol}$

PBS/NEM: $500 \mu \mathrm{L}$ of NEM was added into $50 \mathrm{~mL}$ ice-cold PBS

Ubiquitination Buffer with SDS: $50 \mathrm{mM}$ Tris-Cl pH 7.5 at $4^{\circ} \mathrm{C}, 300 \mathrm{mM} \mathrm{NaCl}, 1 \%$ Triton X100, 0.2 mM PMSF, $1 \mu \mathrm{g} / \mathrm{mL}$ Aprotinin, $0.5 \mu \mathrm{g} / \mathrm{mL}$ Leupeptin, $10 \mathrm{mM}$ NEM

Ubiquitination Buffer with Triton X-100: $50 \mathrm{mM}$ Tris- $\mathrm{Cl} \mathrm{pH} 7.5$ at $4^{\circ} \mathrm{C}, 300 \mathrm{mM} \mathrm{NaCl}, 1 \%$ Triton X-100, 0.2 mM PMSF, $1 \mu \mathrm{g} / \mathrm{mL}$ Aprotinin, $0.5 \mu \mathrm{g} / \mathrm{mL}$ Leupeptin, $10 \mathrm{mM}$ NEM

Washing Buffer: $50 \mathrm{mM}$ Tris-Cl pH 7.5 at $4^{\circ} \mathrm{C}, 300 \mathrm{mM} \mathrm{NaCl}, 1 \%$ Triton X-100, $0.2 \mathrm{mM}$ PMSF, $1 \mu \mathrm{g} / \mathrm{mL}$ Aprotinin, $0.5 \mu \mathrm{g} / \mathrm{mL}$ Leupeptin, $10 \mathrm{mM}$ NEM

\subsection{Electrophysiology}

\subsubsection{Buffers and Solutions}

Sucrose-Based Slicing Solution: $230 \mathrm{mM}$ Sucrose, $26 \mathrm{mM} \mathrm{NaHCO} 3,2 \mathrm{mM} \mathrm{KCl,} 1 \mathrm{mM}$ $\mathrm{KH}_{2} \mathrm{PO}_{4}, 1 \mathrm{mM} \mathrm{MgCl} 2 \times 6 \mathrm{H}_{2} \mathrm{O}, 10 \mathrm{mM}$ Glucose, $0.5 \mathrm{mM} \mathrm{CaCl}_{2}$

Artificial Cerebrospinal Fluid (ACSF) Solution: $120 \mathrm{mM} \mathrm{NaCl}, 26 \mathrm{mM}$ NaHCO3, $1 \mathrm{mM}$ $\mathrm{KH}_{2} \mathrm{PO}_{4}, 2 \mathrm{mM} \mathrm{KCl}, 2 \mathrm{mM} \mathrm{MgCl}{ }_{2} \times 6 \mathrm{H}_{2} \mathrm{O}, 10 \mathrm{mM}$ Glucose, $2 \mathrm{mM} \mathrm{CaCl} 2$

Kainate Solution: 100 nM Kainate in ACSF Solution

\subsubsection{Preparation of Acute Brain Slices}

Postnatal day 22-25 (P22-25) mice were anesthetized using Isofluorane (DeltaSelect) and decapitated. Brains were removed from skull and transverse brain sections with $300 \mu \mathrm{m}$ 
thickness were prepared from both hemispheres using a Leica VT1200S vibratome in ice-cold Sucrose-Based Slicing Solution saturated with carbogen gas (95\% oxygen, 5\% carbon dioxide). Brain slices were transferred to holding chamber filled with ACSF Solution saturated with carbogen gas and incubated for 20 minutes before recording.

\subsubsection{Measurement of Gamma Oscillations ( $\gamma$-Oscillations)}

Gamma-oscillations upon $100 \mathrm{nM}$ kainate application were recorded in the CA3 region of the hippocampus using interface recording chamber (BSCBU Base Unit with the BSC-HT Haas Top, Harvard Apparatus) with constant flow of ACSF at $33^{\circ} \mathrm{C}$. Briefly, extracellular recording electrodes (with a resistance between 2.0-3.0 M $\Omega$ ) filled with ACSF Solution were placed on the CA3 pyramidal cell layer of slices perfused constantly with ACSF Solution and baseline recordings were performed before kainate application for 15 minutes. The oscillatory recordings were performed for 25 minutes upon 100 nM Kainate Solution perfusion. Next, electrodes were re-positioned to find the maximum oscillations and slices were recorded for additional 25 minutes with Kainite Solution application. All measurements were performed using a 700B amplifier (Axon Instruments, Molecular Devices) and Digidata 1440A data acquisition system (Axon Instruments, Molecular Devices) with a Bessel filter at $3 \mathrm{kHz}$. Data analysis were performed using Axograph X software (Axon Instrumetns). The power spectrum from each traces were calculated for 10-minutes epochs. The baseline power spectrum was subtracted from the power spectrum of kainite-induced oscillatory recording. Frequency at maximum power peak, average power, area power and maximum power were determined between $25-45 \mathrm{~Hz}$ oscillatory frequency band.

\subsection{Statistics}

Statistical analyses were done using the Graphpad Prism 5 (Graphpad Software). A statistical significant was concluded when p-value was less than 0.05. In statistical analysis, unpaired, two-tailed Student`s T-test were performed. Variance is expressed as the standard error of mean (SEM). 
3 RESULTS

\subsection{Functions of Nedd4 subfamily E3 ligases in the developing and adult brain}

\subsubsection{Nedd4 family E3 ligases in astrocytes play important roles in neuronal network function}

\subsubsection{Proteome screening identified Kir4.1, Connexin43, and Prr7 as substrates of Nedd4-1 and Nedd4-2 in the mouse brain}

There have been several reports on the screening and identification of substrate proteins of E3 ligases (e.g. using protein chip assays, affinity binding assays, yeast two-hybrid screenings), but the biological relevance of the identified substrate in vivo is often open to dispute. In most of cases, screenings were based on intrinsic biochemical properties of the E3 ligase, such as their high affinities to substrate proteins and their activities to conjugate ubiquitin to substrates. In a high-throughput screening study, multiple potential substrates for human Nedd4-1 (hNedd-1) and human Nedd4-2 (hNedd4-2) have been identified using protein chips (Persaud et al., 2009). In order to identify binding partners for these E3 ligases, recombinant E3 ligases crosslinked with Alexa647 have been incubated on protein chip platform, on which more than 8,000 purified recombinant human proteins were arrayed in native form. In the same study, recombinant E3 ligases were incubated in the presence of E1 enzyme, E2 enzyme, FITC-labelled ubiquitin and ATP on protein chips for in vitro ubiquitination assay. Based on emitted fluorescence from Alexa647 and FITC, binding partners or substrates of Nedd4-1 and Nedd4-2 were identified. Approximately $90 \%$ of E3 ligase binding partners identified in this screening were ubiquitinated by the corresponding E3 enzyme in vitro. One of these binding proteins, FGFR1 was indeed upregulated and protein turnover of FGFR1 was delayed in Nedd4-1 knockdown fibroblasts (Persaud et al., 2011). However, most of the identified substrates contain PPXY motifs, which can be recognized by WW domains of other members of Nedd4 family E3 ligases. Similarly, in a recent proteomics screening study, candidates of HRD1 E3 ligase substrates were identified using siRNA mediated knockdown of HRD1 in cultured HeLa cells (Lee et al., 2011). Briefly, HeLa cells were co-tranfected with His-tagged ubiquitin and siRNA for HRD1 E3 ligase knockdown. Ubiquitination levels of proteins in cell lysates were analyzed using stable isotope labelling by amino acids in cell culture (SILAC) based quantitative mass spectrometry and any proteins showing a reduction in ubiquitination level were reported as substrates of HRD1 E3 ligase. However, siRNA mediated knockdown studies can be 
confounded by off-target effects which complicates the interpretation of data (Khvorova et al., 2003; Lin et al., 2005). Thus, in order to avoid artifacts and obtain more reliable results, it is of particular importance to identify substrates of a E3 ligases using tissue isolated from E3 ligase knockout and control mice.

It has been reported that Nedd4-1 and Nedd4-2 have a preference to form K63-linked polyubiquitin chains (Maspero et al., 2013), which are involved in the endocytosis of transmembrane substrate proteins. Given that Nedd4-1/Nedd4-2 are localized at synapses (Scudder et al., 2014; Kawabe et al., 2010), we hypothesized that in the absence of E3 ligases, endocytosis of relevant substrate proteins would be perturbed and substrate proteins of Nedd4 E3 ligases on synaptic membrane would be upregulated in the brain specific Nedd4-1 and Nedd4-2 double conditional KO (Nedd4-1/f/f; $N e d d 4-2^{\mathrm{fff} f} ;$ Emxl-Cre ${ }^{+/-}$; Nedd4-1/2 bDKO) mice

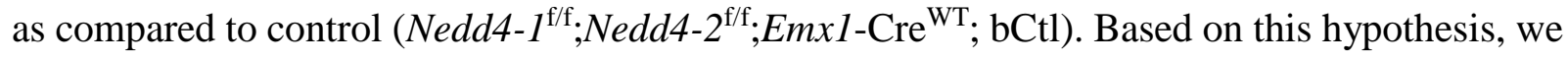
performed quantitative and comparative mass spectrotmetry. For this purpose, mouse cortices from Nedd4-1/2 bDKO and bCtl animals were homogenized in 0.32 $\mathrm{M}$ sucrose and subjected to sucrose gradient ultracentrifugation to isolate pure synaptic plasma membranes (Figure 31A). After the first ultracentrifugation, osmotic shock with $8 \mathrm{mM}$ Tris-Cl, $\mathrm{pH} 8.0$ was applied to synaptosome fractions (P2C) harvested from the interphase between $1.0 \mathrm{M}$ and $1.2 \mathrm{M}$ sucrose layers in order to disrupt the membrane. Later, crude synaptic membrane fractions (CSM) were sedimented by additional centrifugation, where crude synaptic vesicle/synaptic cytoplasm fractions (SC/CSV) were harvested as the supernatant (Figure 3-1B). CSM fractions were further subjected to sucrose gradient ultracentrifugation and pure synaptic membrane fractions (SM3) were harvested from the interphase between 1.0 M and 1.2 M sucrose layers (Figure 31C). Each purified subfraction was validated by Western blotting for PSD-95 and Rab-GDI. In CSM and SM3 fraction, PSD95, a marker for the postsynaptic density (PSD) membrane anchored protein, was enriched, whereas Rab-GDI, a cytoplasmic protein, was depleted (Figure 3-2A), indicating that the subcellular fractionation was performed successfully. Validated SM3 fractions from Nedd4-1/2 bDKO and bCtl animals were subjected to SDS-PAGE. All of the proteins in the polyacrylamide gel were digested by trypsin and peptides were labeled with isobaric Tags for Relative and Absolute Quantitation (iTRAQ) as described previously (Schmidt et al., 2013, with Olaf Jahn, MPI-EM, Goettingen). iTRAQ-labeled samples were subjected to LC-MS/MS for peptide mass quantification (Figure 3-2B). In order to compensate for biased 
efficiencies of labeling of certain peptides by iTRAQ116 and iTRAQ117 reagents, we took two strategies. First, we labeled peptides with two combinations of labeling reagents. For the first round of peptide mass quantification, peptides from Nedd4-1/2 bDKO were labeled with iTRAQ116 and the ones from bCtl were with iTRAQ117. The labelling was reversed for the second round of quantification. Second, relative levels of proteins were estimated with at least five peptides from each protein. In this way, an increase in peptide signals arising from biased labeling efficiencies will be diluted by signals from other peptides. Proteins upregulated in Nedd4-1/2 bDKO are shown in Figure 3-2C. Interestingly, we identified two astrocytic membrane proteins, ATP-sensitive inwardly rectifier potassium channel 10 (Kir4.1), and gap junction protein alpha 1 (Connexin-43, Cnx43), and one postsynaptic membrane protein, Proline-rich protein 7 (Prr7), to be upregulated in Nedd4-1/2 bDKO mice. We then validated the upregulation of Kir4.1 and Cnx43 in SM3 and P2C fractions by Western blotting using antibodies against Kir4.1 and Cnx43. As shown in Figures 3-3 to 3-6, both proteins showed dramatic upregulation in Nedd4-1/2 bDKO mice, indicating that Nedd4-1 and Nedd4-2 E3 ligases might be involved in endocytosis of these membrane proteins on the perisynaptic astrocytic end-feet of tripartite synapses.

\subsubsection{The cytoplasmic C-terminal region of Kir4.1 is ubiquitinated by Nedd4-1 and Nedd4-2 E3 ligases}

In order to study whether Nedd4-1/2 E3 ligases have the potential to ubiquitinate Kir4.1, we used purified recombinant Kir4.1 for in vitro ubiquitination experiment using recombinant Nedd4-1 and Nedd4-2 purified from E. coli. The FLAG-tagged cytoplasmic tail of Kir4.1 was expressed in HEK293FT cells and immunopurified using an anti-FLAG antibody coupled to agarose beads. The beads were incubated with recombinant ubiquitin, E1, E2 and E3 enzymes in the presence of ATP for 1 hour at $37^{\circ} \mathrm{C}$ with moderate agitation. Reactions were analyzed by Western blotting using an anti-FLAG antibody. Only in the presence of an E2 enzyme and Nedd4-1 or Nedd4-2 E3 ligase, the FLAG-tagged C-terminus of Kir4.1 showed a delay of migration in SDS-PAGE with a substantial signal at the higher molecular weight, indicating that Kir4.1 is ubiquitinated by Nedd4-1 and Nedd4-2 (Figure 3-3B). The ubiquitination level was stronger when Nedd4-2 was used as an E3 enzyme as compared to Nedd4-1, indicating that Nedd4-2 has a more potent intrinsic activity to ubiquitinate Kir4.1 than Nedd4-1. 


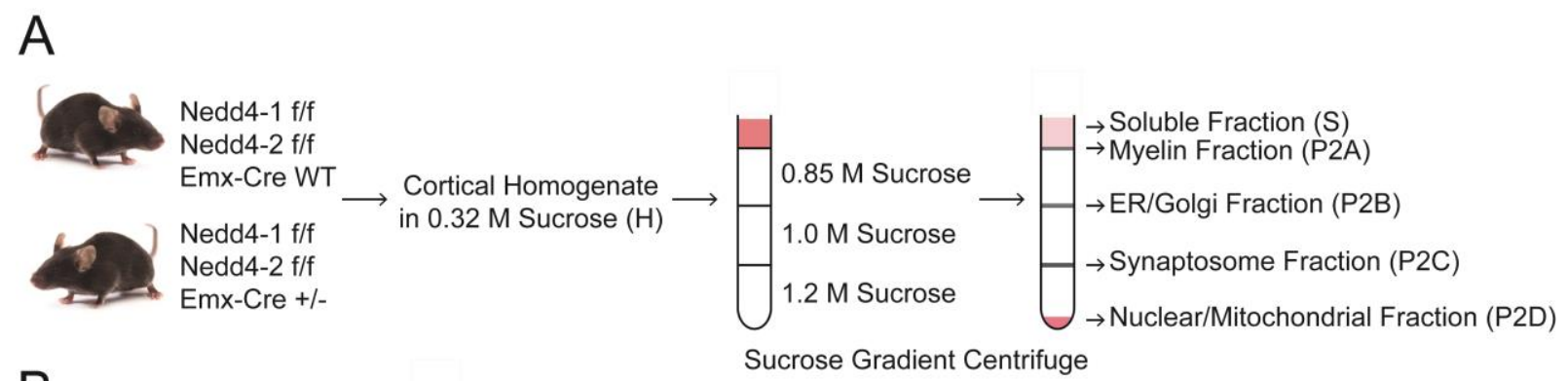

B

Synaptosome Fraction
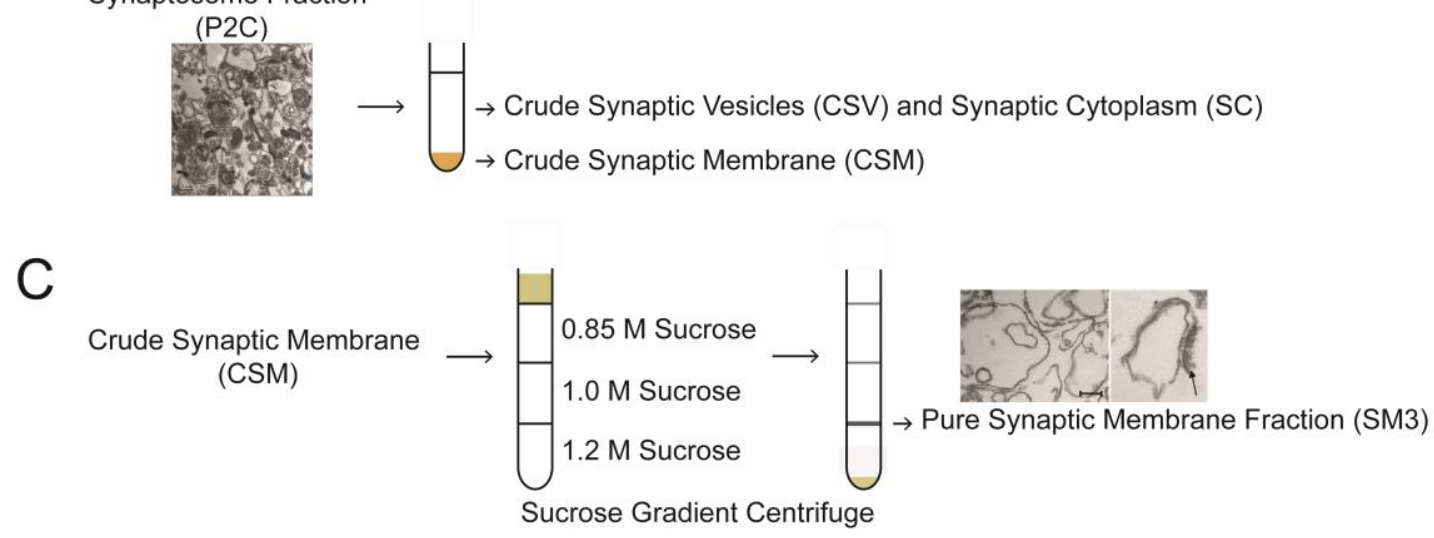

Figure 3-1. Pure synaptic membrane purification

(A) Cortices from Nedd4-1 $1^{\mathrm{fff}} ; \mathrm{Nedd} 4-2^{\mathrm{f} / \mathrm{f}} ; E m x l-\mathrm{Cre}^{+/-}$(Nedd4-1/2 bDKO) and Nedd4-1/f/f; Nedd4-2 ${ }^{\mathrm{f} / \mathrm{f}} ; \mathrm{Em} \times \mathrm{l}$-Cre ${ }^{\mathrm{WT}}$ (bCtl) were homogenized and subjected to discontinuous sucrose gradient ultracentrifugation. The interphase between $1.0 \mathrm{M}$ and $1.2 \mathrm{M}$ sucrose layers was harvested as the synaptosome fraction (P2C). (B) The P2C fraction rich in pre- and postsynaptic components was exposed to osmotic shock to disrupt the synaptic plasma membrane. The sample was further separated into crude synaptic vesicles/synaptic cytoplasm (CSV/SC) and crude synaptic membrane (CSM) fractions by centrifugation (image from Bai and Witzmann, 2007). (C) The CSM fraction was then applied to another round of sucrose density gradient ultracentrifugation in order to purify and enrich the synaptic plasma membrane (SM3) fraction at the interphase between 1.0 M and 1.2 M sucrose layer (image from Frykman et al., 2010). 


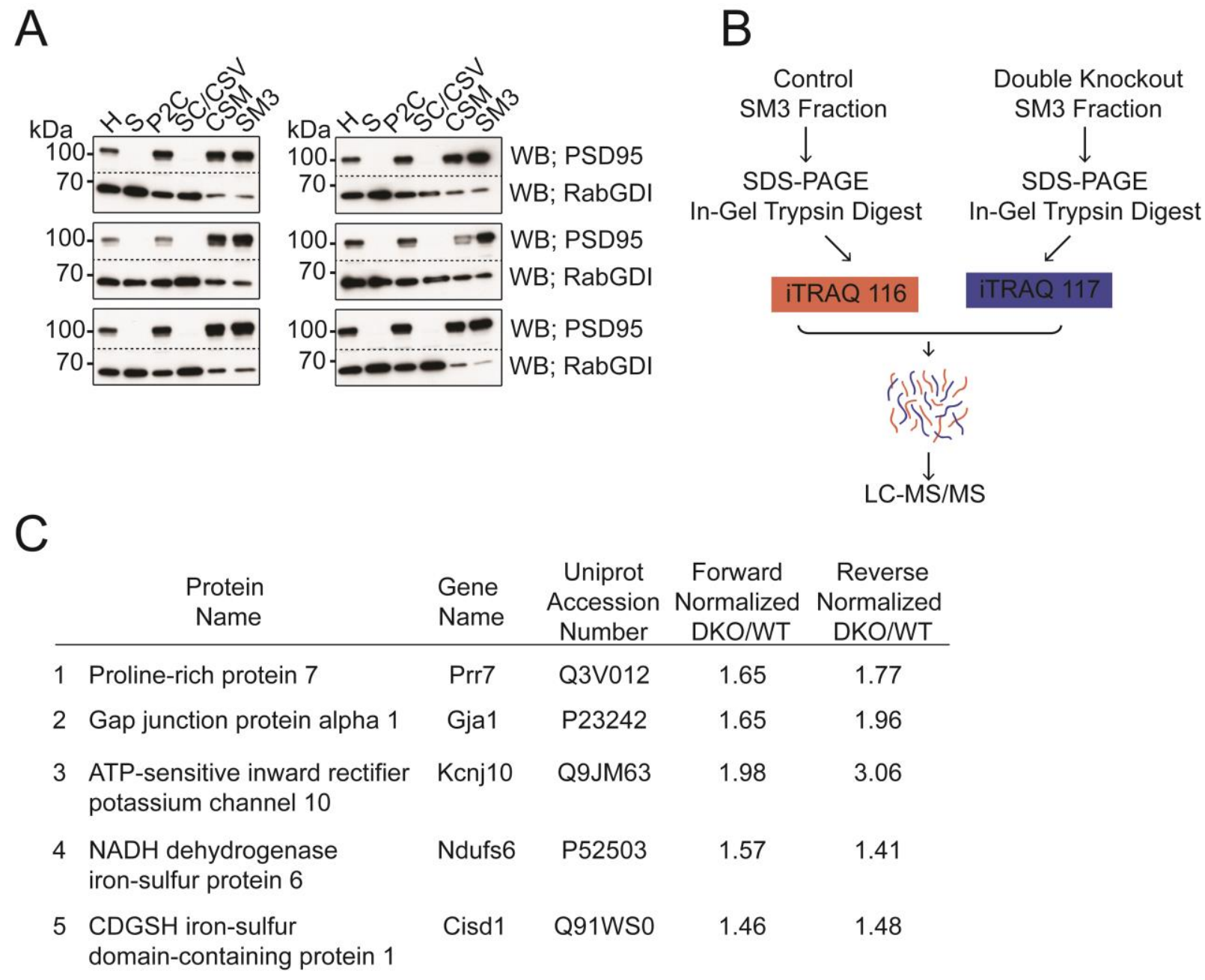

Figure 3-2. Quantitative mass spectroscopic analysis of SM3 fractions using iTRAQ

Validation of purified SM3 fractions. $3 \mu \mathrm{g}$ of each subcellular fraction were analyzed by Western blotting using antibodies against PSD-95 as a postsynaptic marker and against RabGDI as cytoplasmic marker on the same membrane. Note that PSD-95 was enriched in the SM3 fraction whereas Rab-GDI was depleted. (B) Workflow of iTRAQ labelling of SM3 samples. Validated SM3 fractions from Nedd4-1/2 bDKO and bCtl mice were loaded on SDS-PAGE and separated proteins in the polyacrylamide gel were digested with trypsin for iTRAQ labelling. Labelled peptides were analyzed by LC-MS/MS. (C) List of proteins upregulated in Nedd4-1/2 bDKO mice compared to bCtl mice.

\subsubsection{Full-length Kir4-1 is ubiquitinated in vivo by Nedd4-1 and Nedd4-2 E3 ligases}

To test if full-length Kir4.1 is ubiquitinated by Nedd4-1 and Nedd4-2 in vivo, we performed a cell-based ubiquitination assay. We overexpressed Kir4-1 with a C-terminal EGFPtag together with FLAG-tagged Nedd4-1 or Nedd4-2 in HEK293FT cells. Cells were treated with NEM to inhibit de-ubiquitinating enzymes prior to the extraction. Proteins were extracted a lysis buffer containing $1 \%$ SDS and the cell lysate was incubated at $65{ }^{\circ} \mathrm{C}$ for 20 min to 
denature proteins. After diluting the SDS by Triton X100-containing buffer, recombinant Kir4.1 was immunoprecipitated from cell lysates using GFP-trap agarose beads. Ubiquitination levels of Kir4-1 were examined by Western blotting using an anti-ubiquitin antibody. Indeed, Kir4-1 was ubiquitinated highly when Nedd4-1 or Nedd4-2 were co-expressed (Figure 3-3C). The increase in the ubiquitination signal was also apparent in Western blotting using an anti-GFP antibody for immunoprecipitated samples (Figure 3-3D). As seen in the top panel in Figure 33C, expression of Nedd4-1 was more robust than Nedd4-2 expression in this experiment. Nevertheless, the signals cross-reacting with the anti-ubiquitin antibody were comparable between the first and fourth lanes in Figure 3-3C in which FLAG-Nedd4-1 or FLAG-Nedd4-2 were co-expressed with EGFP-Kir4.1, indicating that Nedd4-2 has a stronger activity to ubiquitinate Kir4.1 than Nedd4-1. Thus, consistent with the in vitro ubiquitination assay, fulllength Kir4-1 is ubiquitinated by both Nedd4-1 and Nedd4-2 in vivo, preferentially by Nedd42.

A

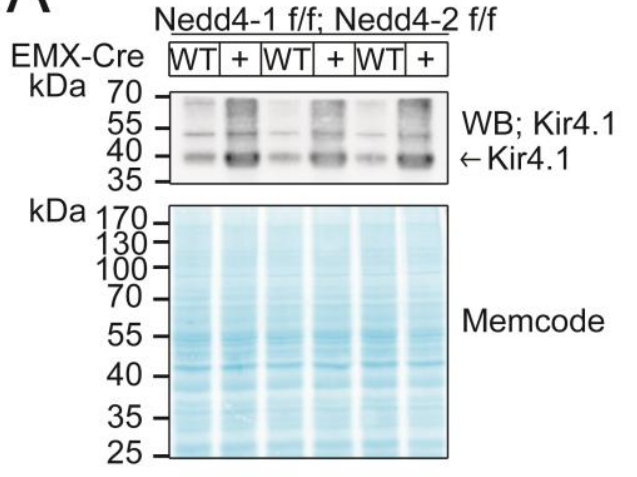

C

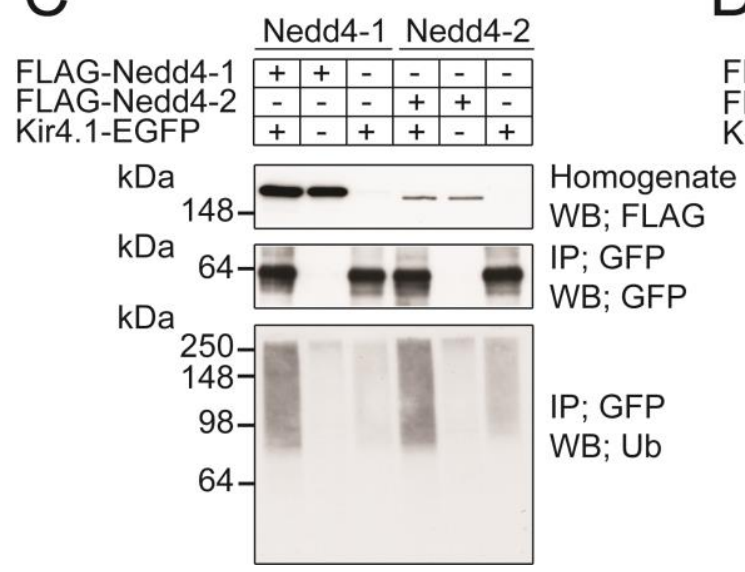

B

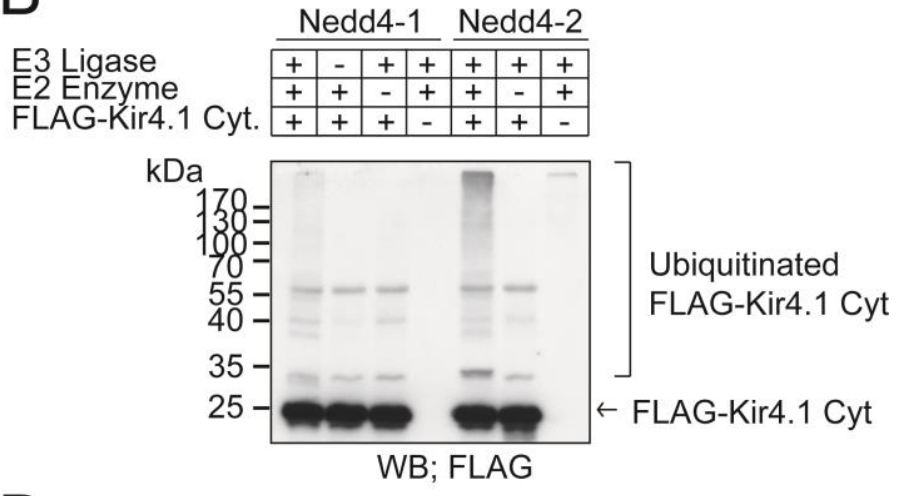

D

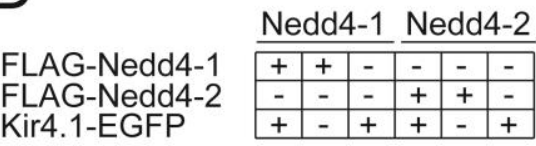

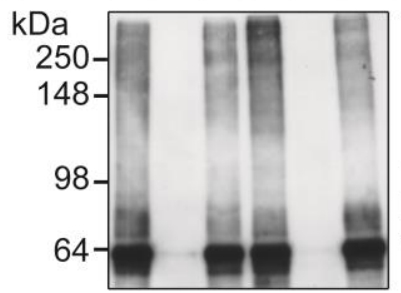

Ubiquitinated

Kir4-1-EGFP

IP; GFP

WB; GFP 
Figure 3-3. Ubiquitination of Kir4.1 by Nedd4-1 and Nedd4-2 mediated ubiquitination Validation of mass spectrometry data of the upregulation of Kir4.1 in Nedd4-1/2 bDKO mice. $10 \mu \mathrm{g}$ of purified SM3 fractions from Nedd4-1/2 bDKO and bCtl mice were loaded on an SDSPAGE gel and analyzed by Western blotting using an anti-Kir4.1 antibody. Signals from proteins stained by Memcode were used as loading controls. Note that Kir4-1 was dramatically upregulated in Nedd4-1/2 bDKO SM3 samples (EMX-Cre +) as compared to bCtl (EMX-Cre WT). Specific band for Kir4.1 is indicated by the arrow. (B) In vitro ubiquitination of Kir4.1 by Nedd4-1 and Nedd4-2 E3 ligases. FLAG-tagged C-terminal cytoplasmic tail of Kir4.1 (FLAGKir4.1 Cyt) was overexpressed in HEK293FT cells and purified using anti-FLAG agarose beads. Subsequently, the proteins on beads were used as substrates for the in vitro ubiquitination assay. Note the shift of FLAG-Kir4.1 Cyt to the range of the high molecular weight with a smear pattern only in the presence of both E2 and E3 enzymes (first lane for Nedd4-1 and fifth lane for Nedd4-2). (C and D) In vivo ubiquitination of Kir4.1 by Nedd4-1 and Nedd4-2 E3 ligases. Full-length Kir4.1 with a C-terminal EGFP tag (Kir4.1-EGFP) was overexpressed with FLAGNedd4-1 or FLAG-Nedd4-2 in HEK293FT cells and immunoprecipitated using GFP-trap agarose beads. The ubiquitination level of Kir4.1-EGFP was analyzed by Western blotting using an anti-ubiquitin antibody. The smear pattern of ubiquitinated Kir4.1-EGFP was increased when Kir4.1-EGFP was overexpressed with FLAG-Nedd4-1 or FLAG-Nedd4-2 (first and fourth lanes) as compared to negative controls. Note that the expression level of FLAG-Nedd4-2 in input samples was weaker than the expression level of FLAG-Nedd4-1 but ubiquitinated Kir4.1EGFP smear patterns were comparable for Nedd4-1 and Nedd4-2. Ubiquitination of Kir4-1EGFP was also apparent in Western blotting using an anti-GFP antibody for immunoprecipitated samples. Kir4-1-EGFP showed stronger smear patterns when co-expressed with FLAG-Nedd41 and FLAG-Nedd4-2 in GFP Western blot.

\subsubsection{Nedd4-2 is a physiological E3 ligase for Kir4.1}

Upon ubiquitination by an E3 ligase, transmembrane proteins undergo endocytosis with the help of adaptor proteins (e.g. epsin and Eps15). Endocytosed transmembrane proteins can be either recycled to the plasma membrane or degraded in lysosomes. To test, which of these effects is caused by Nedd4 subfamily E3 ligases, we studied the levels of Kir4.1 in cerebral homogenates. Our study was based on the hypothesis that a lack of lysosomal degradation would lead to the accumulation of a substrate protein in the entire cell while reduced recycling will result in an accumulation of a substrate solely on the plasma membrane. Interestingly, we detected 5.3-fold upregulation of Kir4.1 in homogenates from 6 week-old Nedd4-1/2 bDKO mice compared to 6 week-old Nedd4-1/2 bCtl mice (Figure 3-4A: Control $1.0 \pm 0.02, \mathrm{~N}=3$; DKO $5.28 \pm 0.29, \mathrm{~N}=3 ; \mathrm{p}=0.0001$ ), indicating that ubiquitination of Kir4.1 by Nedd4-1 and Nedd4-2 E3 ligases plays a role in lysosomal degradation rather than recycling.

Nedd4-1/2 bDKO mice were generated by crossing $N e d d 4-1^{\mathrm{f} / \mathrm{f}} ; N e d d 4-2^{\mathrm{f} / \mathrm{f}}$ mice with Emxl-Cre mice. In Emxl-Cre driven lines, the Cre recombinase is expressed in glutamergic 
neurons, astrocytes, and oligodendrocytes (Gorski et al., 2002). In order to test if the upregulation of Kir4.1 in Nedd4-1/2 bDKO is indeed due to the absence of Nedd4-1 and Nedd42 activity in glia cells, we tested the levels of Kir4.1 in cerebral homogenates from 6 weeks-old Nedd4-1 ${ }^{\mathrm{f} / \mathrm{f}} ; N e d d 4-2^{\mathrm{f} / \mathrm{f}} ; N E X 1-\mathrm{Cre}^{+/-}(\mathrm{Nedd} 4-1 / 2 \quad \mathrm{nDKO})$ and 6-week-old Nedd4-1 ${ }^{\mathrm{f} / \mathrm{f}} ; N e d d 4-$ $2^{\mathrm{f} / \mathrm{f}} ; N E X-\mathrm{Cre}^{\mathrm{WT}}(\mathrm{nCtl})$ animals. In Nedd4-1/2 nDKO, Cre recombinase is expressed mainly in postmitotic neurons but not in glia cells (Goebbels et al., 2006). Interestingly, the levels of Kir4.1 in Nedd4-1/2 nDKO animals were not changed compared to nCtl mice (Figure 3-4B: $\mathrm{nCtl}, 1.00 \pm 0.04, \mathrm{~N}=3 ; \mathrm{Nedd} 4-1 / 2 \mathrm{nDKO}, 1.03 \pm 0.09, \mathrm{~N}=3 ; \mathrm{p}=0.7685)$, indicating that the knockout Nedd4-1 and Nedd4-2 specifically in glia cells leads to an upregulation of Kir4.1 in glia cells.

Since Nedd4-2 showed stronger ubiquitination of Kir4.1 in ubiquitination assays in vivo and in vitro, we tested the Kir4.1 levels in Nedd4-1 ${ }^{\mathrm{f} / \mathrm{f}} ;$ Emxl-Cre $^{+/-}$single knockout (Nedd4-1 bKO) and Nedd4-2/f/f Emxl-Cre ${ }^{+/-}$single knockout mice (Nedd4-2 bKO). We found a 2.7-fold upregulation of Kir4.1 levels in cortical homogenates of Nedd4-2 bKO mice as compared to samples from control Nedd4-2 $2^{f / f}(\mathrm{Nedd} 4-2 \mathrm{bCtl})$ mice (Figure 3-4C: Nedd4-2 bCtl, $1.00 \pm 0.16$, $\mathrm{N}=3$; Nedd4-2 bKO, $2.64 \pm 0.04, \mathrm{~N}=3 ; \mathrm{p}=0.0006$ ). However, the level of Kir4.1 was not changed in cortical homogenates from Nedd4-1 bKO as compared to samples from control Nedd4-1 f/f (Nedd4-1 bCtl) mice (Figure 3-4D: Control, $1.00 \pm 0.18, \mathrm{~N}=4$; Nedd4-1 bKO, $0.92 \pm 0.10, \mathrm{~N}=4$; $\mathrm{p}=0.7364$ ). Based on these data, we conclude that Nedd4-2 predominantly ubiquitinates Kir4.1 in vivo, leading to its lysosomal degradation, even though Nedd4-1 has the potential to ubiquitinate Kir4.1.

\subsubsection{Connexin-43 is ubiquitinated by Nedd4-1 and Nedd4-2 E3 Ligases in vivo}

Connexin-43 was identified in the proteome screening to be upregulated proteins in Nedd4-1/2 bDKO as compared to Nedd4-1/2 bCtl. Indeed, Connexin-43 has been reported as a binding partner of Nedd4-1 in an in vitro binding assay, where the PPXY motif of Connexin-43 bound to the second WW domain of Nedd4-1 (Kawabe et al., 2010; Leykauf et al., 2006). In the cultured cells line, the level of Connexin-43 was significantly upregulated upon knock-down of endogenous Nedd4-1 (Girão et al., 2009). We validated the result of proteome screening by Western blotting for Connexin-43 in P2C fractions from Nedd4-1/2 bDKO and Nedd4-1/2 bCtl 
mice (Figure 3-5A). In order to test if Connexin-43 is ubiquitinated by Nedd4-1 and Nedd4-2 E3 ligases, we performed a cell based ubiquitination assay. C-terminally HA-tagged Connexin43 (Connexin-43-HA) was co-expressed with EGFP-Nedd4-1 and EGFP-Nedd4-2 in HEK293FT cells and immunoprecipitated using an anti-HA antibody coupled to agarose beads with the same protocol as used for Kir4-1-EGFP. Later, ubiquitination levels of Connexin-43HA were studied by Western blotting using an anti-ubiquitin antibody. Interestingly, the signal from the anti-ubiquitin antibody at high molecular weight was increased when EGFP-Nedd4-1 or EGFP-Nedd4-2 were co-expresesd (Figure 3-5B), indicating that Connexin-43-HA is ubiquitinated by EGFP-Nedd4-1 and EGFP-Nedd4-2 in vivo. The level of ubiquitinated Connexin-43-HA was higher when co-expressed with EGFP-Nedd4-2 than with EGFP-Nedd41, although the expression level of EGFP-Nedd4-2 was weaker than level of EGFP-Nedd4-1.

Next, we investigated the type of polyubiquitin chains conjugated to Connexin- 43 by Nedd4-1 and Nedd4-2 E3 ligases. For this purpose, the immunoprecipitated samples (Figure 35B) were subjected to Western blotting using antibodies specific for K63- or K48-linked polyubiquitin chains. In order to compare the signals from two anti-ubiquitin antibodies directly, $25 \mathrm{ng}$ of recombinant K48-linked and K63-linked tetrapeptides (BostonBiochem) were loaded on the SDS-PAGE gels together with immunoprecipitated samples. X-ray films for Western blotting were exposed so that signals from K63-linked and K48-linked tetrapeptides using the respective antibodies were comparable. When co-expressed with Nedd4-1 or Nedd4-2, the ubiquitinated Connexin-43-HA smear pattern was only increased upon K63 chain-specific ubiquitin Western blotting but not upon K48 chain-specific Western blotting as compared to negative controls (Figure 3-5C). Interestingly, the signal from the K48-linked polyubiquitin chain-specific antibody was most prominent when Connexin-43-HA was overexpressed without E3 ligase. We propose that this is due to increased removal of overexpressed Connexin-43-HA by endoplasmic reticulum-associated degradation (ERAD) where K48-linked polyubiquitin chain plays a crucial role (Lemus and Goder, 2014). Taken together, we conclude here that Nedd4-1 and Nedd4-2 conjugate Connexin-43 with K63-linked polyubiquitin chains and thereby target this transmembrane protein for degradation. 

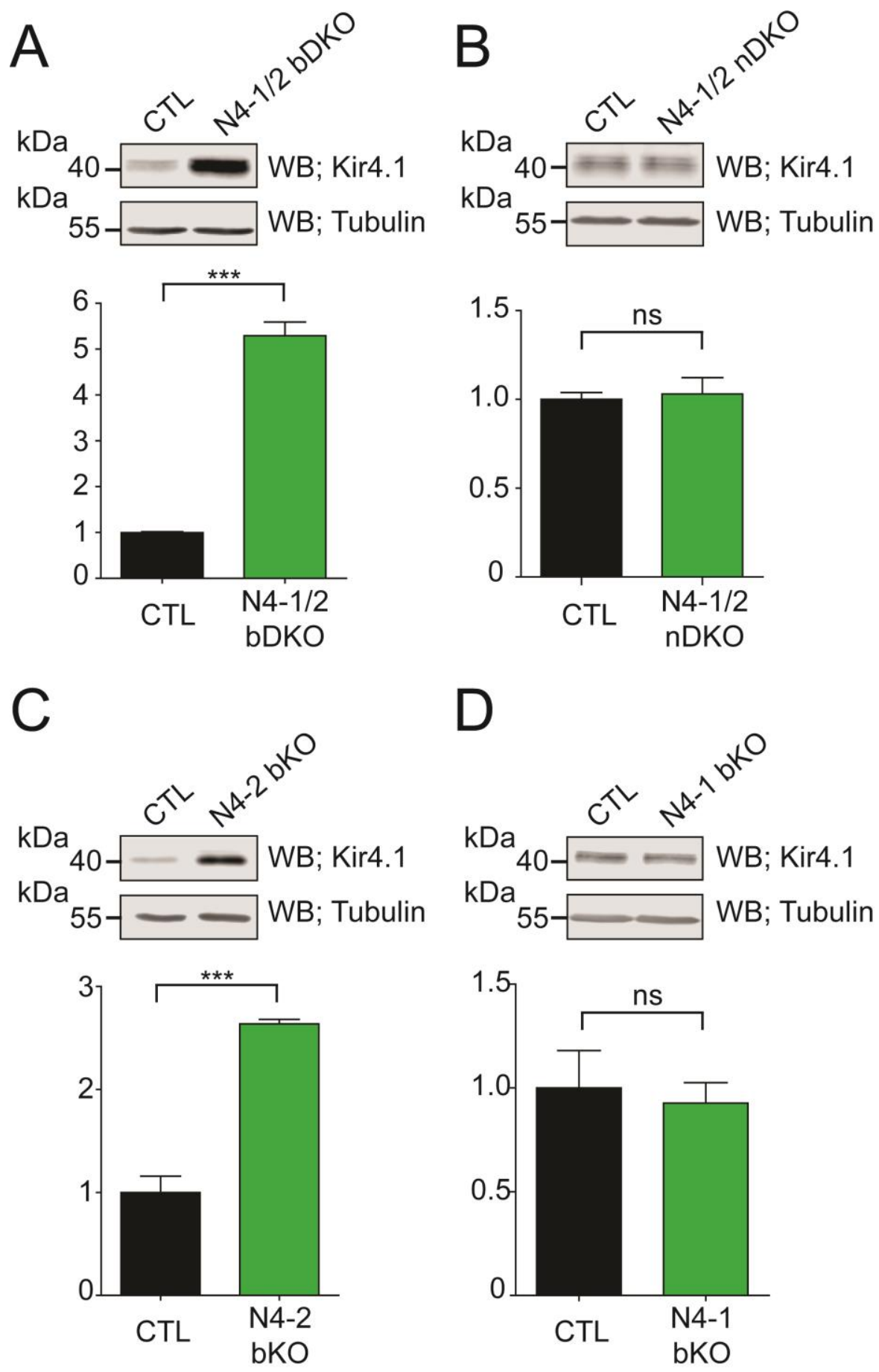

Figure 3-4. Kir4.1 levels in different Nedd4-1 and Nedd4-2 conditional knockout lines Cortical homogenates from 6 weeks-old conditional knockout and control animals were subjected to Western blotting using an anti-Kir4.1 antibody (upper panels) and an anti-tubulin antibody (bottom panels). Relative Kir4.1 levels were quantified by normalizing the signal from the anti-Kir4.1 antibody to that from the antibody against tubulin. Bar diagrams represent mean \pm SEM. (A) Kir4.1 is upregulated in Nedd4-1/2 bDKO cortical homogenates. Nedd4-1/2 bCtl, $1.0 \pm 0.02, \mathrm{~N}=3$; Nedd4-1/2 bDKO, $5.28 \pm 0.29, \mathrm{~N}=3 ; \mathrm{p}=0.0001$. (B) Kir4.1 levels are not 
changed in Nedd4-1/2 nDKO cortical homogenates. Nedd4-1/2 nCtl $1.00 \pm 0.04, \mathrm{~N}=3$; Nedd4$1 / 2 \mathrm{nDKO}, 1.03 \pm 0.09, \mathrm{~N}=3 ; \mathrm{p}=0.7685$. (C) Kir4.1 levels are upregulated in cortical homogenates from Nedd4-2 bKO. Nedd4-2 bCtl $1.00 \pm 0.16$, N=3; Nedd4-2 bKO, $2.64 \pm 0.04$, $\mathrm{N}=3 ; \mathrm{p}=0.0006$. (D) Kir4.1 protein levels are not changed in Nedd4-1 bKO cortical homogenates. Nedd4-1 bCtl, $1.00 \pm 0.18, \mathrm{~N}=4$; Nedd4-1 bKO, $0.92 \pm 0.10, \mathrm{~N}=4 ; \mathrm{p}=0.7364$.

A

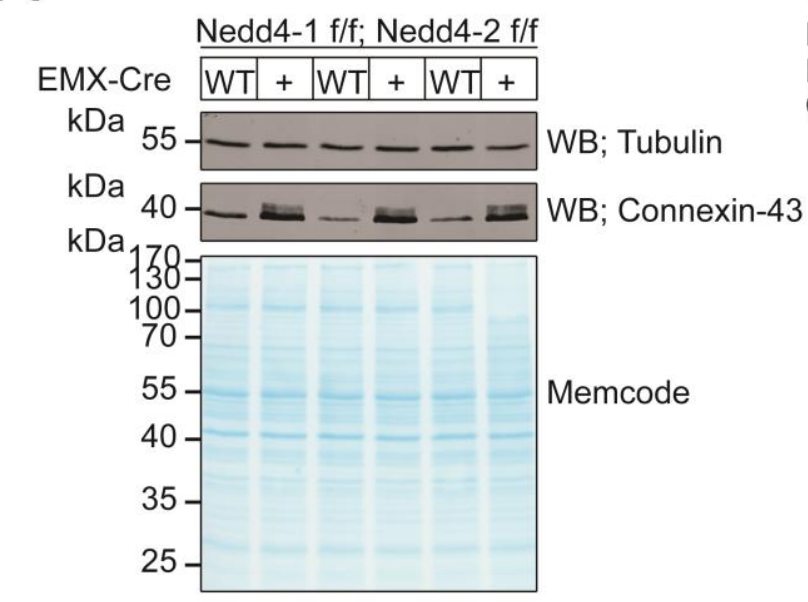

C
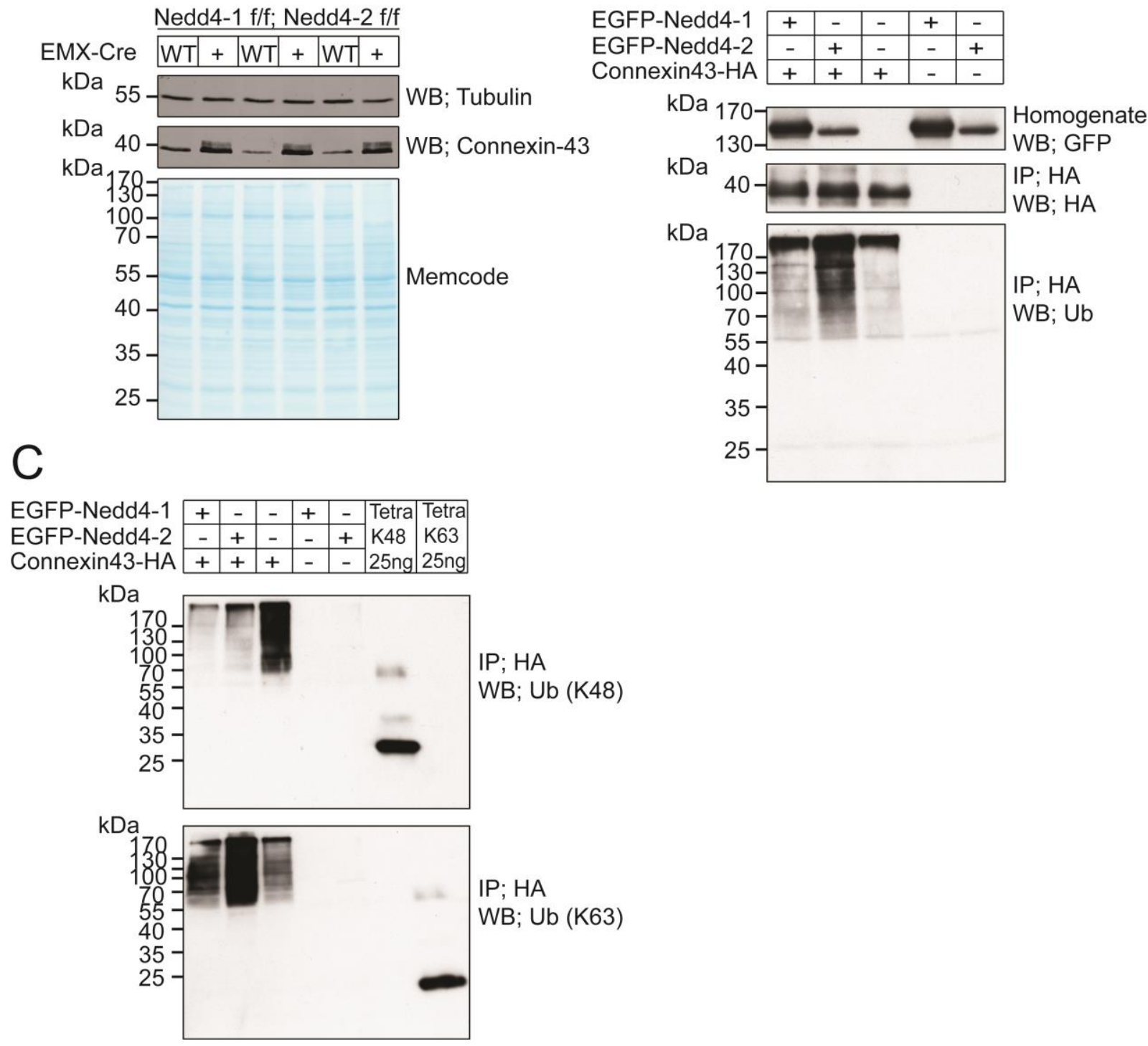

Figure 3-5. Ubiquitination of Connexin-43 by Nedd4-1 and Nedd4-2

Validation of proteome screening by Western blotting with an anti-Connexin-43 antibody. $8 \mu \mathrm{g}$ of P2C fractions from Nedd4-1/2 bDKO and Nedd4-1/2 bCtl mice were analyzed by Western blotting using antibodies against tubulin (upper panel) and Connexin-43 (middle panel) after memcode staining (bottom panel). (B) Connexin-43 is ubiquitinated in vivo by Nedd4-1 and Nedd4-2 E3 ligases. C-teminally HA-tagged Connexin-43 (Connexin-43-HA) was co-expressed with EGFP-Nedd4-1 or EGFP-Nedd4-2 in HEK293FT cells and immunoprecipitated using anti- 
HA agarose beads. Ubiquitination levels were analyzed by Western blotting using an antiubiquitin antibody. The signal from the anti-ubiquitin antibody was increased when Connexin43 was co-expressed with Nedd4-1 or Nedd4-2 (first and second lanes) as compared to negative controls (third to fifth lane). (C) Nedd4-1 and Nedd4-2 ubiquitinate Connexin-43 with K63 polyubiquitin chains. After co-expression with Nedd4-1 and Nedd4-2, Connexin-43-HA was immunoprecipitated and analyzed by Western blotting using antibodies specific to K48-linked or K63-linked polyubiquitin chains. Note that signals from $25 \mathrm{ng}$ of K48-linked and K63-linked tetrapeptides in top and bottom panels are comparable. Nedd4-1- and Nedd4-2-dependent increases in the signals were observed only when blotted with a K63-linked polyubiquitin specific antibody (bottom panel), but not with a K48-linked polyubiquitin specific antibody (top panel).

\subsubsection{Connexin-43 levels are regulated by Nedd4-1 and Nedd4-2 mediated ubiquitination}

Given that Connexin-43 is ubiquitinated by Nedd4-1 and Nedd4-2 with K63-linked polyubiquitin chains and that the Connexin-43 level is upregulated in the $\mathrm{P} 2 \mathrm{C}$ fraction of the Nedd4-1/2 bDKO, we tested Connexin-43 levels in cortical homogenates in order to assess if the ubiquitination of Connexin-43 leads to lysosomal degradation. Cortical homogenates from 6 week-old Nedd4-1/2 bDKO and Nedd4-1/2 bCtl mice were prepared and analyzed by Western blotting using an anti-Connexin-43 antibody. We detected a 1.95-fold upregulation of Connexin-43 in cortical homogenates of Nedd4-1/2 bDKO as compared to control cortical homogenates (Figure 3-6A: Nedd4-1/2 bCtl $1.00 \pm$ 0.035, N=3; Nedd4-1/2 bDKO 1.95 \pm 0.16, $\mathrm{N}=3 ; \mathrm{p}=0.0044$ ). This result indicates that ubiquitination of Connexin-43 by Nedd4-1 and Nedd4-2 plays a role in the endocytosis and lysosomal degradation of Connexin- 43.

Given that Connexin-43 is expressed mainly in astrocytes (Dere et al., 2012), we next tested if Connexin-43 upregulation in cortical homogenates of Nedd4-1/2 bDKO is due to the loss of function of Nedd4-1 and Nedd4-2 in astrocytes or due to a secondary effect from the loss of Nedd4-1 and Nedd4-2 in neurons. Cortical homogenates from 6 week-old Nedd4-1/2 nDKO and control mice were analyzed by Western blotting using an anti-Connexin-43 antibody. Indeed, the level of Connexin-43 was not changed in cortical homogenates of Nedd4-1/2 nDKO as compared to control cortical homogenates (Figure 3-6B: Nedd4-1/2 nCtl $1.00 \pm 0.012, \mathrm{~N}=3$; Nedd4-1/2 nDKO $1.051 \pm 0.04, \mathrm{~N}=3 ; \mathrm{p}=0.29)$, indicating that upregulation of Connexin-43 in Nedd4-1/2 bDKO is mainly caused in a cell-autonomous fashion by the loss of function of Nedd4-1 and Nedd4-2 in astrocytes. 

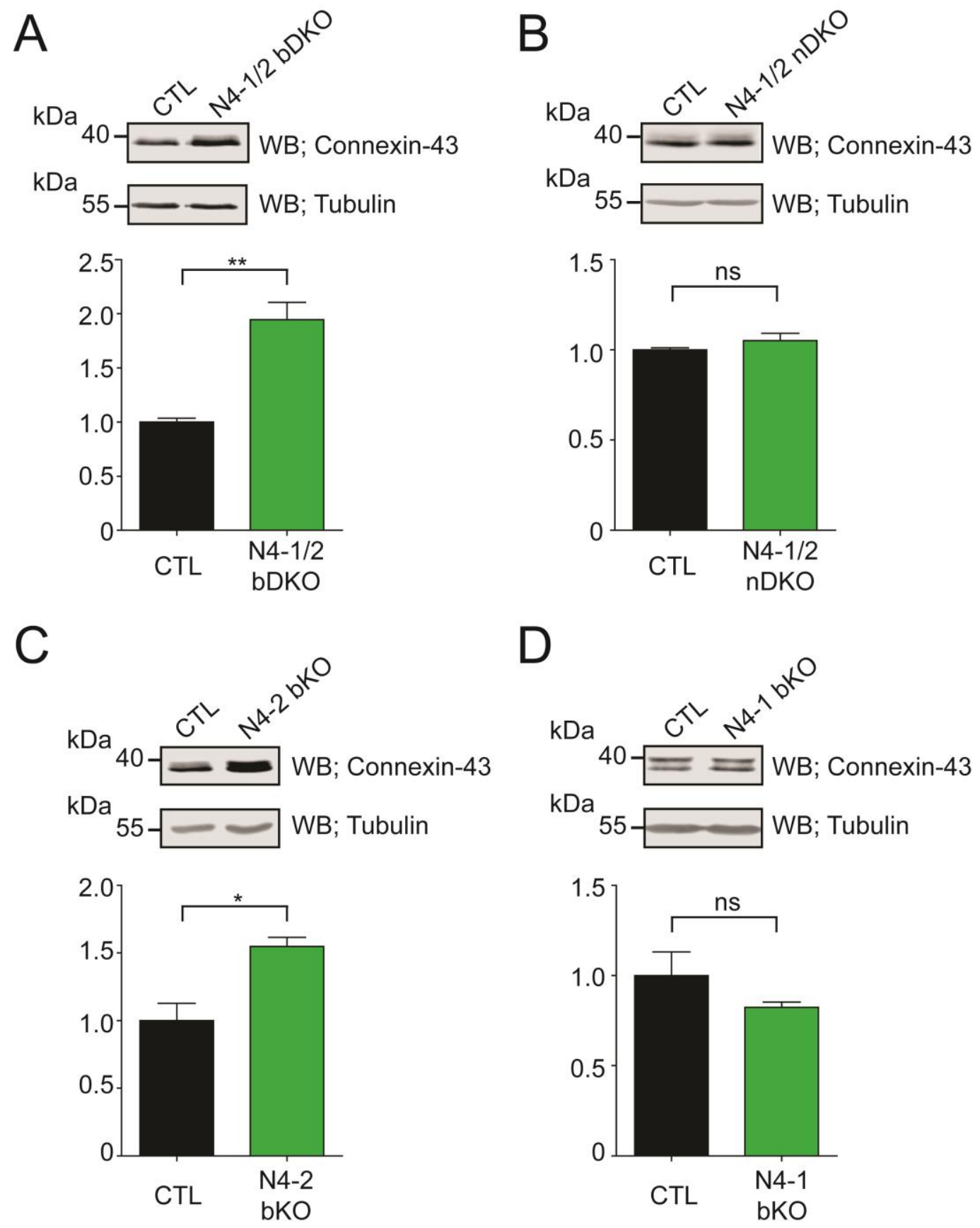

Figure 3-6. Connexin-43 levels in different Nedd4-1 and Nedd4-2 conditional knockout lines

Cortical homogenates from 6 weeks-old conditional knockout and control animals were analyzed by Western blotting using an anti-Connexin-43 antibody (upper panels), and an antitubulin antibody (bottom panels). Relative Connexin-43 levels were quantified by normalizing the signal from the anti-Connexin-43 antibody to the signal from the anti-tubulin antibody. Bar diagrams represent mean \pm SEM. (A) Connexin- 43 is upregulated in Nedd4-1/2 bDKO cortical homogenates. Nedd4-1/2 bCtl, $1.00 \pm 0.035, \mathrm{~N}=3$; Nedd4-1/2 bDKO, $1.95 \pm 0.16 \mathrm{~N}=3 ; \mathrm{p}=$ 0.0044. (B) Connexin-43 levels are not changed in Nedd4-1/2 nDKO cortical homogenates. Nedd4-1/2 nCtl $1.00 \pm 0.012, \mathrm{~N}=3$; Nedd4-1/2 nDKO, $1.051 \pm 0.040, \mathrm{~N}=3 ; \mathrm{p}=0.2927$. (C) 
Connexin-43 levels are upregulated in cortical homogenates from Nedd4-2 bKO. Nedd4-2 bCtl $1.00 \pm 0.13, \mathrm{~N}=3$; Nedd4-2 bKO, $1.55 \pm 0.068, \mathrm{~N}=3 ; \mathrm{p}=0.0193$. (D) Connexin-43 protein levels are not changed in Nedd4-1 bKO cortical homogenates. Nedd4-1 bCtl, $1.00 \pm 0.13, \mathrm{~N}=4$; Nedd4$1 \mathrm{bKO}, 0.82 \pm 0.029, \mathrm{~N}=4 ; \mathrm{p}=0.2382$.

Given the fact that Nedd4-2 has a more potent activity to conjugate K63-linked polyubiquitin chains to Connexin-43, we analyzed the levels of Connexin-43 in cortical homogenates from 6 week-old Nedd4-1 bKO and Nedd4-2 bKO as well as control mice. As shown in Figure 3-6C, Connexin-43 was upregulated 1.55-fold in cortical homogenates from Nedd4-2 bKO compared to cortical homogenates from control mice (Nedd4-2 bCtl 1.00 \pm 0.128 , $\mathrm{N}=3$; Nedd4-2 bKO $1.55 \pm 0.068, \mathrm{~N}=3 ; \mathrm{p}=0.019)$ but the level of Connexin-43 was not changed in Nedd4-1 bKO cortical homogenates compared to control mice (Figure 3-6D: Nedd4-1 bCtl $1.00 \pm 0.13 \mathrm{~N}=4$; Nedd4- 1 bDKO $0.82 \pm 0.029 \mathrm{~N}=4$; $\mathrm{p}=0.238$ ). Thus, ubiquitination and levels of Connexin-43 in vivo are mainly regulated by Nedd4-2.

\subsubsection{Hippocampal circuitry in Nedd4-1/2 bDKO mice is imbalanced}

Connexin-43 is a gap junction protein expressed in astrocytes, where it plays a crucial role in mediating astrocytic ICWs (Soroceanu et al., 2001; Yamamoto et al., 1992), and together with Kir4.1, Connexin-43 is involved in spatial potassium buffering (Neusch et al., 2001). Thus, Connexin-43 might coordinate synchronization of neuronal functions through the astrocytic network. Given that Connexin-43 and Kir4.1 levels are upregulated in Nedd4-1/2 bDKO as compared to control mice, we hypothesized that neuronal networks might be imbalanced in the hippocampus of Nedd4-1/2 bDKO mice. Gamma oscillations indicate neuronal synchronization, and kainate-induced gamma oscillations in the CA3 region of the hippocampus show an ageddependent reduction (Lu et al., 2011; Vreugdenhil and Toescu, 2005).

In order to test our hypothesis, we measured kainate-induced gamma oscillations in the CA3 pyramidal layer of the hippocampus in 3 week-old Nedd4-1/2 bDKO and control mice. Before the measurement of gamma oscillations, we checked if the expression levels of Kir4.1 and Connexin-43 are perturbed in 3 week-old Nedd4-1/2 bDKO and bCtl mice. Cortical homogenates from 3 week-old Nedd4-1/2 bDKO and bCtl animals were analyzed by Western blotting using anti-Kir4.1 and anti-Connexin-43 antibodies. Figures 3-7A to 3-7B show that Kir4.1 and Connexin-43 levels are upregulated in three week-old Nedd4-1/2 bDKO mice as compared to Nedd4-1/2 bCtl mice, to a similar extend as seen in 6 week-old animals. We next 
recorded gamma oscillations in the CA3 region of hippocampal brain slices prepared from P21P24 mice. Extracellular field potentials were recorded from the CA3 pyramidal layer of the hippocampus during constant perfusion of ACSF as baseline recordings. Gamma oscillation was induced by ACSF containing $100 \mathrm{nM}$ Kainate. After baseline subtraction, the averaged power of gamma oscillations between $25-45 \mathrm{~Hz}$ frequencies were analyzed. Gamma oscillatory activity in Nedd4-1/2 bDKO was dramatically reduced in comparison to control mice (Figures 3-8A to 3-8D). The averaged power of gamma oscillations in control mice was $333.0 \pm 72.82 \mu \mathrm{V}^{2} / \mathrm{Hz}$ (16 slices, 3 animals) whereas that in Nedd4-1/2 bDKO mice was $99.10 \pm 24.42 \mu \mathrm{V}^{2} / \mathrm{Hz}(21$ slices, 3 animals, $\mathrm{p}=0.0018$ ). The frequency of gamma oscillations, however, was not changed in the absence of Nedd4-1 and Nedd4-2 (Nedd4-1/2 bCtl, $32.04 \pm 0.66 \mathrm{~Hz}$; Nedd4-1/2 bDKO, $33.20 \pm 0.77 \mathrm{~Hz} ; \mathrm{p}=0.28$ ) (Figure 3-8 E).


Figure 3-7. Kir4.1 and Connexin-43 levels are upregulated in 3 weeks-old Nedd4-1/2 bDKO cortical homogenates

Cortical homogenates from 3 weeks-old conditional knockout and control animals were analyzed by Western blotting using anti-Kir4.1, anti-Connexin-43 (upper panels) and antitubulin antibodies (bottom panels). Relative Kir4.1 and Connexin-43 levels were quantified by normalizing the signals from the anti-Kir4.1 or anti-Connexin-43 antibody to the signal from the anti-tubulin antibody. Bar diagrams represent mean \pm SEM. (A) Kir4.1 levels are upregulated in cortical homogenates from Nedd4-1/2 bDKO as compared to control. Nedd4-1/2 bCtl, $1.00 \pm 0.19 \mathrm{~N}=3$; Nedd4-1/2 nDKO, $7.30 \pm 0.30, \mathrm{~N}=3 ; \mathrm{p}<0.0001$. (B) Levels of Connexin- 
43 are upregulated in Nedd4-1/2 bDKO cortical homogenates as compared to control cortical homogenates. Nedd4-1/2 bCtl, $1.00 \pm 0.13, \mathrm{~N}=3$; Nedd4-1/2 nDKO, $2.943 \pm 0.06, \mathrm{~N}=3$; $\mathrm{p}=0.0002$.

A

Before Kainate

Nedd4-1/f/f; Nedd4-2 $2^{\mathrm{ftf}}$

\lrcorner $0.1 \mathrm{mV}$
$60 \mathrm{msec}$

Before Kainate

Nedd4-1 ${ }^{\mathrm{fff}} ; \mathrm{Nedd} 4-2^{\mathrm{fff}} ; \mathrm{EMX}-\mathrm{Cre}^{+/-}$
B

After Kainate (100 nM)

Nedd4-1/f/f; Nedd4-2

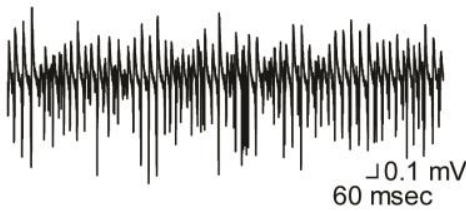

After Kainate (100 nM) Nedd4-1 ${ }^{\mathrm{ff} / \mathrm{F}}$ : Nedd4-2 ${ }^{\mathrm{fff}} ; \mathrm{EMX}-\mathrm{Cre}^{+/-}$

\lrcorner $0.1 \mathrm{mV}$
$60 \mathrm{msec}$

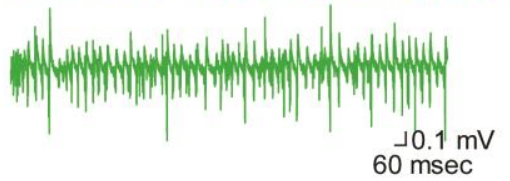

C

- Nedd4-1/f/f; Nedd4-2 $2^{\mathrm{f} / \mathrm{f}}$

- Nedd4-1/ff; Nedd4-2f/f; EMX-Cre ${ }^{+/-}$

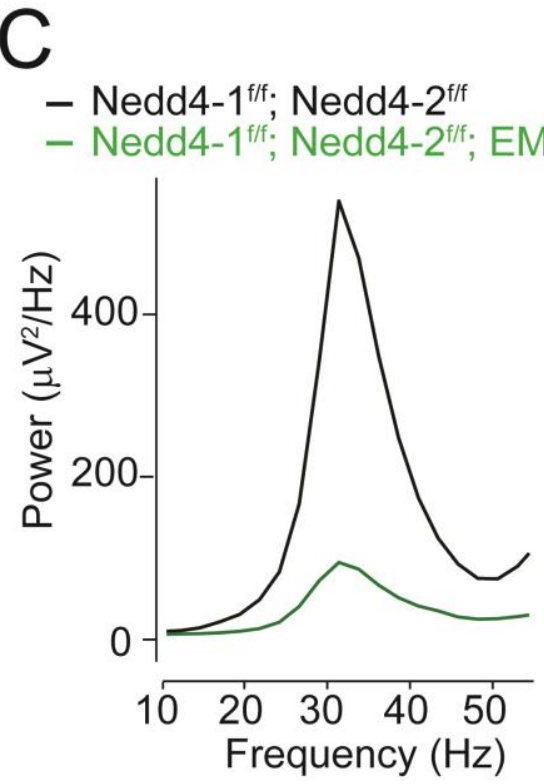

D

$E$

\lrcorner $0.1 \mathrm{mV}$
$60 \mathrm{msec}$
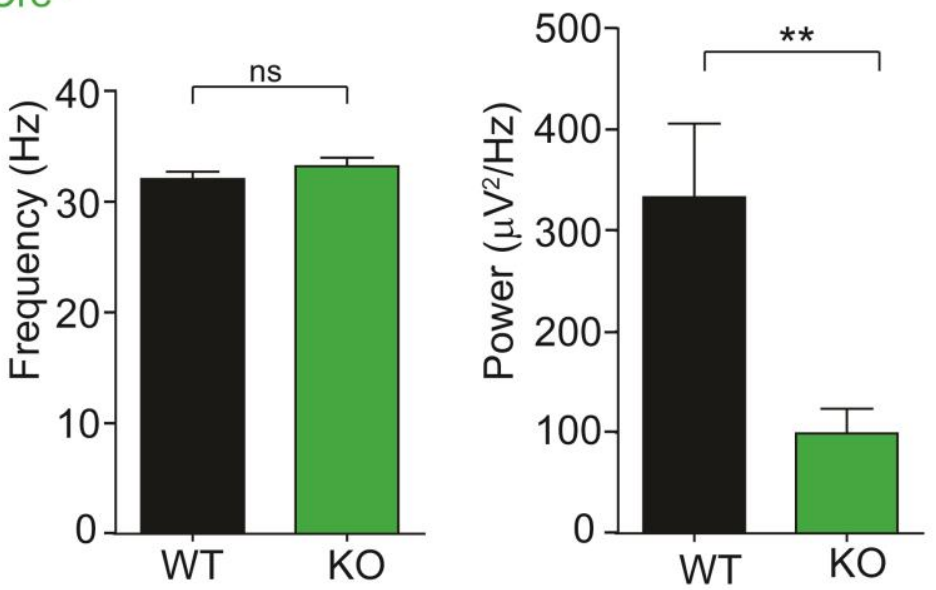

Figure 3-8. Nedd4-1/2 bDKO mice show reduced gamma oscillations in the CA3 pyramidal layer of hippocampus

Representative traces of gamma oscillations recorded in the CA3 pyramidal layer of the hippocampus before the kainate application in Nedd4-1/2 bCtl (upper traces) and Nedd4-1/2 bDKO (lower traces) (B) Representative traces of gamma oscillations after $100 \mathrm{nM}$ kainate application in control (upper traces) and Nedd4-1/2 bDKO mice (lower traces). Note the reduced power of oscillations in Nedd4-1/2 bDKO (C) Representative power spectrum of gamma oscillations induced by $100 \mathrm{nM}$ kainate application in brain slices from Nedd4-1/2 bCtl and Nedd4-1/2 bDKO mice. The power of gamma oscillations is reduced in Nedd4-1/2 bDKO as 
compared to control mice. (D) Frequency of gamma oscillations is not changed in Nedd4-1/2 $\mathrm{bDKO}$ as compared to control mice. Mean frequency of gamma oscillations at maximum peak in power spectrum is shown in the bar diagram $( \pm \mathrm{SEM})$. Nedd4-1/2 bCtl, $32.04 \pm 0.66, \mathrm{~N}=3$, $\mathrm{n}=16$; Nedd4- $1 / 2 \mathrm{bDKO}, 33.20 \pm 0.77, \mathrm{~N}=3, \mathrm{n}=21 ; \mathrm{p}=0.28$. (E) Averaged power of gamma oscillations is reduced in Nedd4-1/2 bDKO as compared to control mice. For each recording, the averaged power of gamma oscillations was analyzed between $25-45 \mathrm{~Hz}$ in 10 min epochs. Bar diagram represents the mean of averaged power of oscillations $( \pm$ SEM). Nedd4-1/2 bCtl $333.0 \pm 72.82, \mathrm{~N}=3, \mathrm{n}=16$; Nedd4 $-1 / 2 \mathrm{bDKO}, 99.10 \pm 24.42 \mathrm{~N}=3, \mathrm{n}=21, \mathrm{p}=0.0018$.

\subsubsection{Altered gamma oscillations in Nedd4-1/2 bDKO are caused by loss of glial Nedd4-}

\section{1 and Nedd4-2}

In Nedd4-1/2 bDKO mice, Nedd4-1 and Nedd4-2 are deleted in postmitotic neurons and glia cells. In the next set of experiments, we investigated if the reduction in gamma oscillatory activity in Nedd4-1/2 bDKO is caused by the loss of Nedd4-1 and Nedd4-2 in glia cells or in neurons. In order to study the impact of the loss of Nedd4 genes in the neuron, we measured gamma oscillations in the CA3 pyramidal layer of the hippocampus in Nedd4-1/2 nCtl and Nedd4-1/2 nDKO mice, where Cre recombinase is expressed only in postmitotic neurons. Interestingly, no significant differences in gamma oscillations were observed in Nedd4-1/2 nDKO as compared to Nedd4-1/2 nCtl mice (Figure 3-9 A-D: Nedd4-1/2 nCtl $114.5 \pm 20.66$, $\mathrm{N}=4, \mathrm{n}=19$; Nedd4-1/2 nDKO $89.53 \pm 16.43, \mathrm{~N}=4, \mathrm{n}=20, \mathrm{p}=0.3474)$, and the frequencies of gamma oscillations were also unchanged in Nedd4-1/2 nDKO mice (Figure 3-9E: Nedd4-1/2 $\mathrm{nCtl}, ; 33.28 \pm 0.78, \mathrm{~N}=4, \mathrm{n}=19 ; \mathrm{Nedd} 4-1 / 2 \mathrm{nDKO}, 34.66 \pm 0.76, \mathrm{~N}=4, \mathrm{n}=20 ; \mathrm{p}=0.2142)$. Taken together, our results indicate that glial Nedd4-1 and Nedd4-2 are involved in neuronal network regulation, likely through the perisynaptic end-feet of astrocytes and the astrocytic network, by regulating the protein levels of Kir4.1 and Connexin-43. 
A

Before Kainate

Nedd4-1 $1^{\mathrm{fff}} ;$ Nedd4- $2^{\mathrm{fff}}$

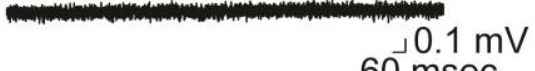

$60 \mathrm{msec}$

Before Kainate

Nedd4-1 ${ }^{\mathrm{flf}} ; \mathrm{Nedd} 4-2^{\mathrm{f} / \mathrm{f}} ; \mathrm{NEX}-\mathrm{Cre}^{+/-}$
B

After Kainate (100 nM)

Nedd4-1/f/f; Nedd4-2/f/

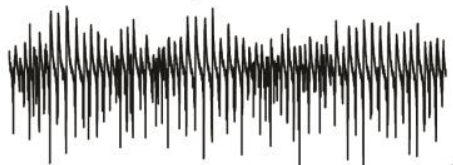

$0.1 \mathrm{mV}$

$60 \mathrm{msec}$

After Kainate (100 nM)

Nedd4-1 ${ }^{\mathrm{fff}} ; \mathrm{Nedd} 4-2^{\mathrm{ff/}} ; \mathrm{NEX}-\mathrm{Cre}^{+/-}$

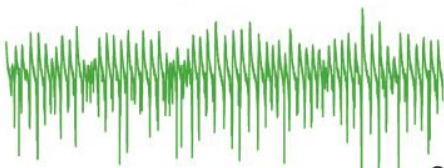

$60 \stackrel{0.1 \mathrm{mV}}{\mathrm{msec}}$

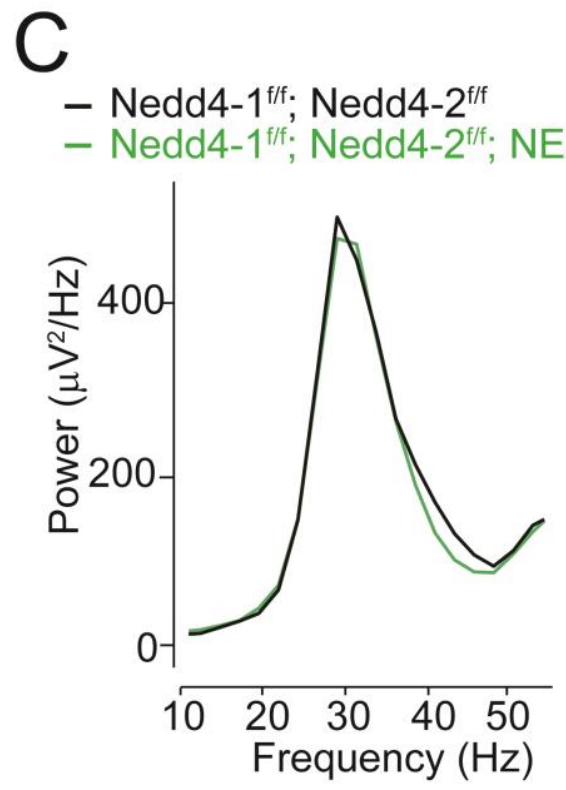

D

E

- Nedd4-1 $1^{\mathrm{f} / \mathrm{f}} ;$ Nedd4-2 $\mathrm{f/f}$

- Nedd4-1/ff; Nedd4-2 ${ }^{\mathrm{fff}} ; \mathrm{NEX}-\mathrm{Cre}^{+/-}$
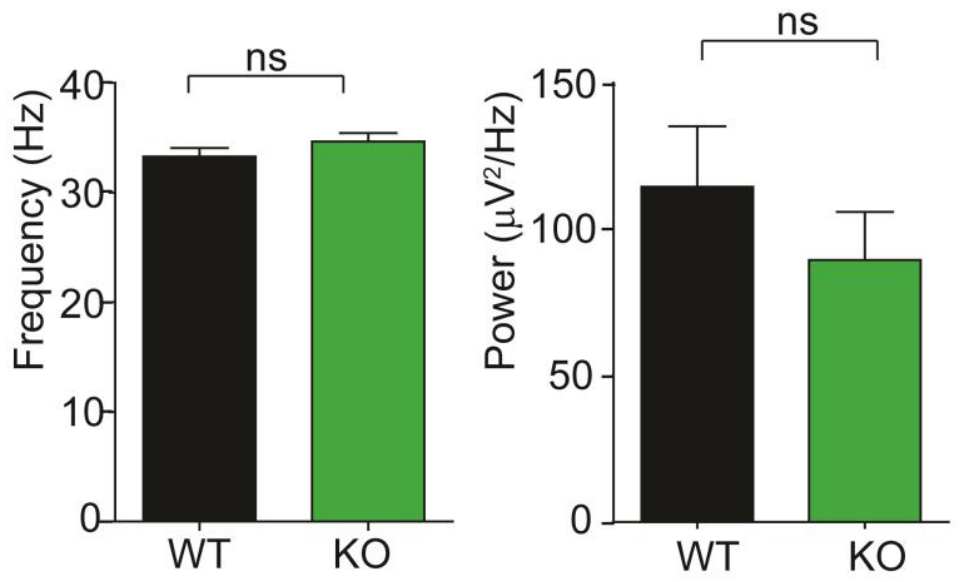

Figure 3-9. Gamma oscillations are not changed in Nedd4-1/2 nDKO mice

Representative traces of gamma oscillations measured in the CA3 region of the hippocampus before the kainate application in Nedd4-1/2 bCtl (upper traces) and Nedd4-1/2 bDKO (lower traces). (B) Representative traces of gamma oscillations after $100 \mathrm{nM}$ kainate application in control (upper traces) and Nedd4-1/2 bDKO mice (lower traces). (C) Representative power spectrum of gamma oscillations in the CA3 pyramidal layer of the hippocampus upon $100 \mathrm{nM}$ kainate application in brain slices from Nedd4-1/2 nCtl and Nedd4-1/2 nDKO mice. The power of gamma oscillations is not changed in Nedd4-1/2 $\mathrm{nDKO}$ as compared to control mice. (D) The frequency of gamma oscillations is not changed in Nedd4-1/2 nDKO as compared to Nedd4-1/2 $\mathrm{nCtl}$ mice. The bar diagram represents the mean frequency of gamma oscillations at the maximum peak in the power spectrum $( \pm \mathrm{SEM})$. Nedd4-1/2 $\mathrm{nCtl} ; 33.28 \pm 0.78, \mathrm{~N}=4, \mathrm{n}=19$; Nedd4-1/2 nDKO, $34.66 \pm 0.76, \mathrm{~N}=4, \mathrm{n}=20 ; \mathrm{p}=0.2142$. (E) The averaged power of gamma 
oscillations is not significantly changed in Nedd4-1/2 nDKO as compared to Nedd4-1/2 nCtl mice. The averaged power of gamma oscillations was analyzed between $25-45 \mathrm{~Hz}$ in 10 min epochs. The bar diagram represents the mean of averaged power of oscillations \pm SEM. Nedd4$1 / 2$ nCtl 114.5 $\pm 20.66, \mathrm{~N}=4, \mathrm{n}=19 ; \mathrm{Nedd} 4-1 / 2$ nDKO $89.53 \pm 16.43 \mathrm{~N}=4, \mathrm{n}=20, \mathrm{p}=0.3474$.

\subsubsection{Nedd4-2 bKO mice show reduced gamma oscillations in the CA3 pyramidal layer of the hippocampus}

Given that Nedd4-2 has a stronger ubiquitination activity towards Connexin-43 and Kir4.1 than Nedd4-1, we tested if Nedd4-2 bKO show reduced gamma oscillatory activity as compared to control mice. We recorded gamma oscillations induced by $100 \mathrm{nM}$ Kainate in the CA3 pyramidal layer within the hippocampus. Remarkably, the power of gamma oscillations was reduced in Nedd4-2 bKO mice as compared to control mice (Figure 3-10A-D: Nedd4-2 bCtl; $258.4 \pm$ 46.48; N=5 Animals, n=26 Slices; Nedd4-2 bKO; $146.3 \pm 26.21$; N=5 Animals, $\mathrm{n}=25$ slices; $\mathrm{p}=0.0430$ ), with no significant difference in the frequency of oscillations (Figure 3-10E: Nedd4-2 bCtl, $31.90 \pm 0.82, \mathrm{~N}=5, \mathrm{n}=26$; Nedd4-2 bKO, $33.83 \pm 0.56 \mathrm{~N}=5, \mathrm{n}=25$; $\mathrm{p}=0.0615)$. Taken together, these data show that Nedd4-2 has a role in the regulation of gamma oscillatory activity in the CA3 pyramidal layer of the hippocampus by regulating the protein levels of Connexin-43 and Kir4.1. 
A

Before Kainate

Nedd4-2 $/ / 4$

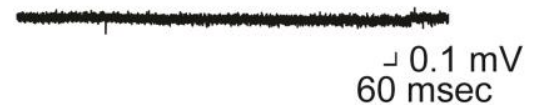

Before Kainate

Nedd4-2/ff; EMX-Cre ${ }^{+/-}$

\lrcorner $0.1 \mathrm{mV}$
B

After Kainate (100 nM)

Nedd4-2 ${ }^{\text {f/f }}$

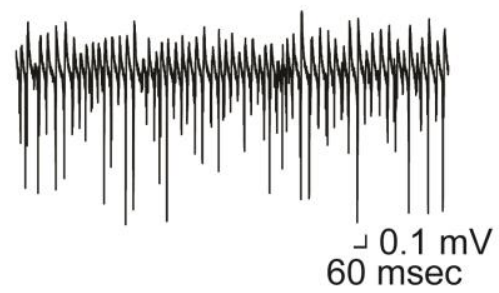

After Kainate (100 nM) Nedd4-2 ${ }^{\mathrm{ft} ;} ; \mathrm{EMX}-\mathrm{Cre}^{+/-}$

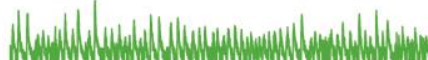

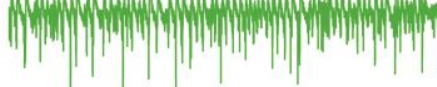

\lrcorner $0.1 \mathrm{mV}$
C

- Nedd4-2 $2^{\mathrm{ft}}$

- Nedd4-2fff; EMX-Cre ${ }^{+/}$

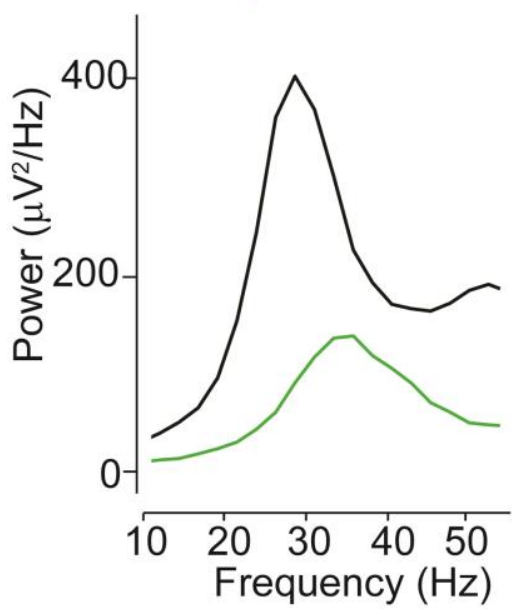

$D$

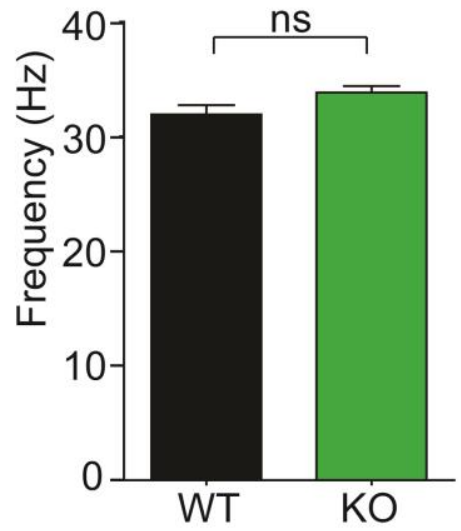

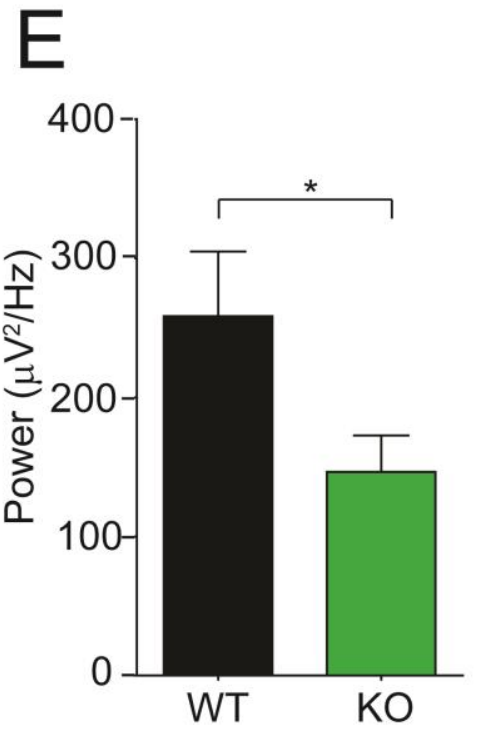

Figure 3-10. Nedd4-2 bKO shows reduced gamma oscillations in CA3 region of hippocampus

(A) Representative traces of gamma oscillations before the kainate application in Nedd4-2 bCtl (upper traces) and Nedd4-2 bKO mice (lower traces) (B) Representative traces of gamma oscillations after $100 \mathrm{nM}$ kainate application in control (upper traces) and Nedd4-2 bKO mice (lower traces). Note the reduced power of oscillations in Nedd4-2 bKO (C) Representative power spectrum of gamma oscillations measured after $100 \mathrm{nM}$ kainate application in brain slices from Nedd4-2 bCtl and Nedd4-2 bDKO mice. (D) Frequency of gamma oscillations is not changed in Nedd4-2 bKO as compared to control mice. The mean frequency of gamma oscillations at the maximum peak in power spectrum were shown in bar diagram $( \pm \mathrm{SEM})$. Nedd4-2 bCtl, $31.90 \pm 0.82, \mathrm{~N}=5, \mathrm{n}=26$; Nedd4-2 bKO, $33.83 \pm 0.56 \mathrm{~N}=5, \mathrm{n}=25 ; \mathrm{p}=0.0615$. (E) 
The averaged power of gamma oscillations is reduced in Nedd4-2 bKO as compared to Nedd4$2 \mathrm{bCtl}$ mice. The averaged power of gamma oscillations was analyzed between $25-45 \mathrm{~Hz}$ in 10 min epochs. The bar diagram represents the mean of averaged power of oscillations \pm SEM. Nedd4-2 bCtl, $258.4 \pm 46.48 \mathrm{~N}=5, \mathrm{n}=26$; Nedd4-2 bKO, $146.3 \pm 26.21 \mathrm{~N}=5, \mathrm{n}=25 ; \mathrm{p}=0.043$.

\subsubsection{Nedd4 subfamily E3 ligases might play a role in spine maturation}

\subsubsection{Prr7 is a substrate of Nedd4-2}

In the proteome screening, we also identified Prr7 to be upregulated in the SM3 fraction of Nedd4-1/2 bDKO mice as compared to Nedd4-1/2 bCtl mice. In order to validate the results of mass spectrometry, we studied the levels of endogenous Prr7 in SM3 fractions from Nedd41/2 bDKO and Nedd4-1/2 bCtl mice by quantitative Western blotting using an anti-Prr7 antibody. As shown in Figure 3-11A, the level of Prr7 was upregulated in Nedd4-1/2 bDKO mice as compared to Nedd4-1/2 bCtl mice. Next, we tested if Prr7 is ubiquitinated by Nedd4-1 or Nedd4-2 in an in vitro ubiquitination assay. Myc-tagged Prr7 was overexpressed in HEK293FT cells, immunopurified using an anti-myc antibody coupled to agarose beads, and used as the substrate for an in vitro ubiquitination assay. Myc-Prr7 was incubated with ATP, recombinant ubiquitin, and E1 enzyme in the presence or absence of E2 or E3 enzymes. Proteins were separated by SDS-PAGE and subjected to Western blotting using an anti-myc antibody. Only in the presence of the E2 enzyme and recombinant Nedd4-2, myc-Prr7 migrated more slowly on SDS-PAGE, showing a smear pattern at high molecular weight, indicating that Prr7 is ubiquitinated by Nedd4-2 (Figure 3-11B).

\subsubsection{Prr7 is conjugated with K63 polyubiquitin chains by Nedd4-1 and Nedd4-2 E3}

\section{ligases in vivo}

In order to test if full length Prr7 is ubiquitinated by Nedd4-1 or Nedd4- 2 in vivo, we performed in vivo ubiquitination assays. Full length Prr7 with a C-terminal FLAG-tag (Prr7FLAG) was co-expressed with EGFP-Nedd4-1 or EGFP-Nedd4-2 in HEK293FT cells. Subsequently, Prr7-FLAG was immunoprecipitated using an anti-FLAG antibodies coupled to agarose beads, employing a similar protocol as used for Kir4.1 and Connexin-43. Ubiquitination levels of Prr7-FLAG were analyzed by Western blotting using three anti-ubiquitin antibodies, anti-pan-ubiquitin, anti-K48-linked polyubiquitin, and anti-K63-linked polyubiquitin antibodies. Signals from the anti-pan-ubiquitin antibody at the high molecular weight range were increased when Prr7-FLAG was co-expressed with EGFP-Nedd4-1 or EGFP-Nedd4-2 as 
compared to negative controls (Figure 3-11C), indicating that Prr7-FLAG is ubiquitinated in vivo by EGFP-Nedd4-1 and EGFP-Nedd4-2. Although the expression level of EGFP-Nedd4-2 was weaker than the level of EGFP-Nedd4-1 in cell homogenates, the level of ubiquitinated Prr7-FLAG was higher when co-expressed with EGFP-Nedd4-2 than with EGFP-Nedd4-1, indicating that Nedd4-2 has stronger intrinsic activity to ubiquitinate Prr7 in vivo than Nedd41. Next, we analyzed the type of polyubiquitin chain conjugated to Prr7 by Nedd4-1 and Nedd42. For this purpose, immunoprecipitated Prr7-FLAG was analyzed by Western blotting using antibodies specific to K48- and K63-linked polyubiquitin chains. Similar to the analysis of Connexin-43-HA, we loaded 25 ng of pure K48-linked and K63-linked tetraubiquitins to SDSPAGE gels for the comparison of titers of the two different anti-ubiquitin antibodies. As shown in Figure 3-11D, EGFP-Nedd4-1- and EGFP-Nedd4-2-dependent increases in signals from immunoprecipitated Prr7-FLAG were observed only when blotted with the K63-linked polyuibuqitin chain-specific antibody; such increases were not observed using the K48-linked polyuibuqitin chain-specific antibody. 
A

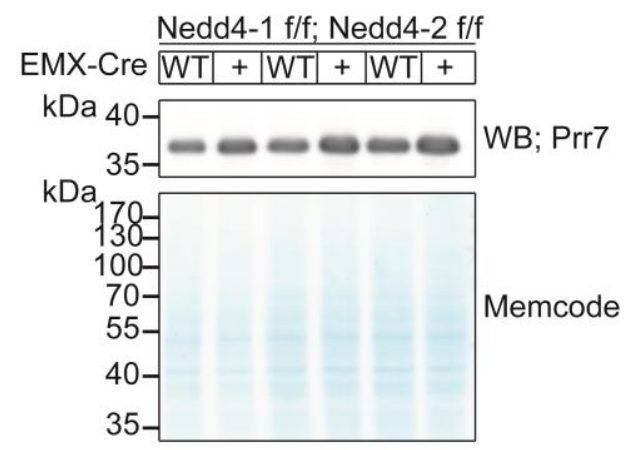

C

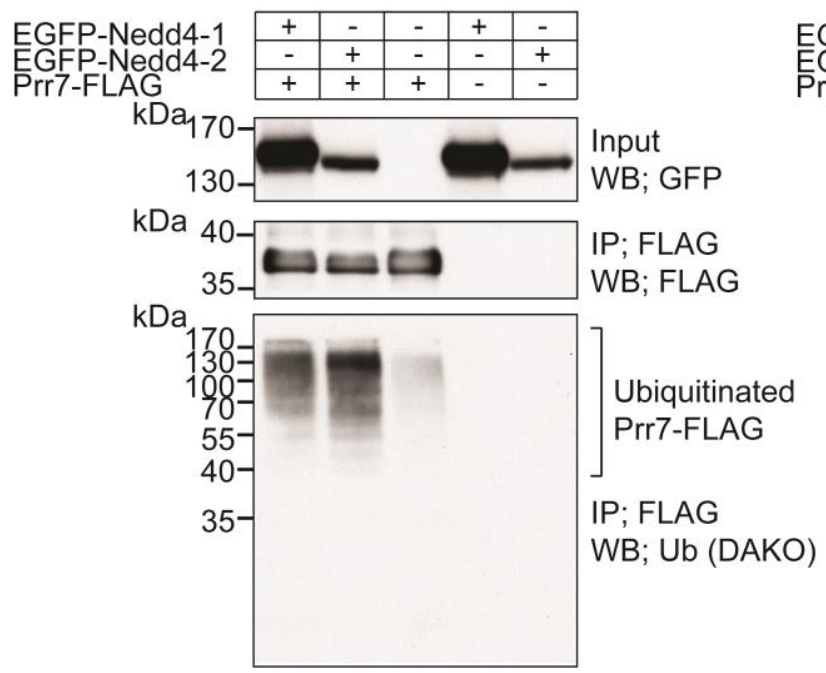

B

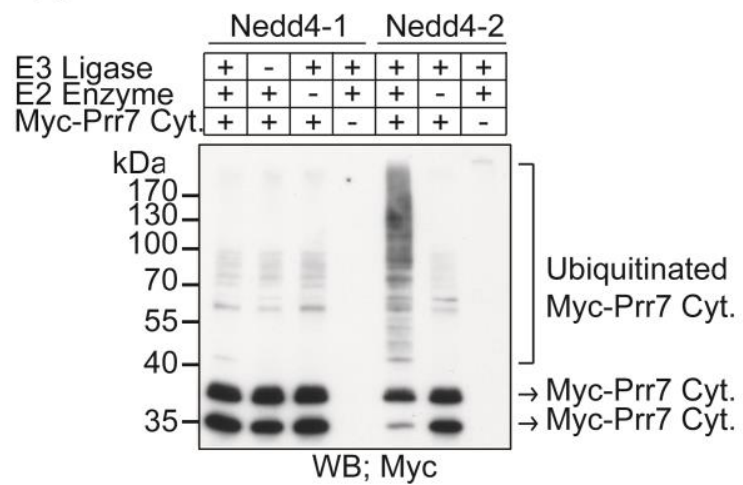

$\mathrm{D}$

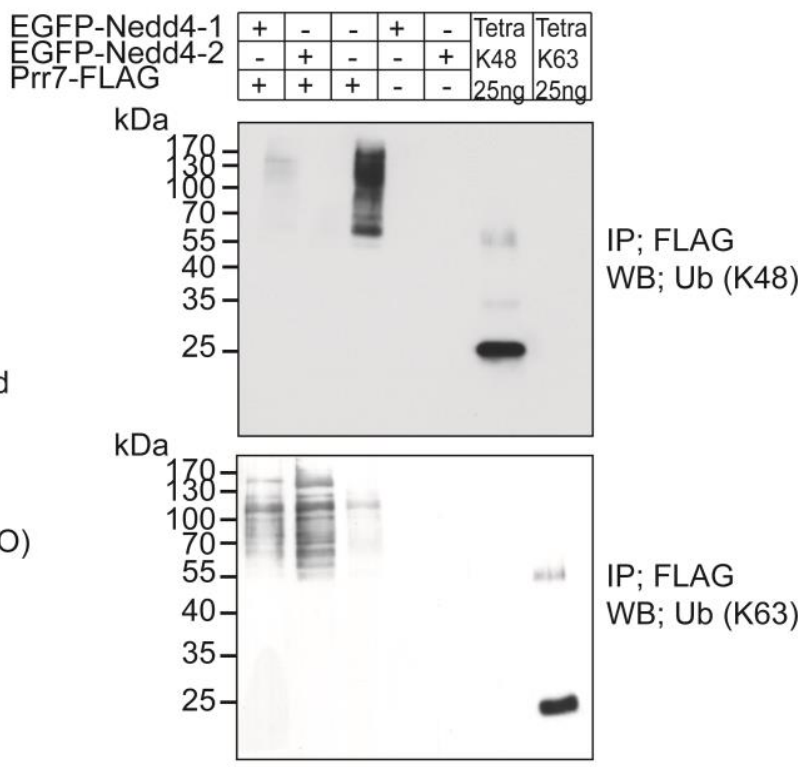

Figure 3-11. Ubiquitination of Prr7 by Nedd4-1 and Nedd4-2 mediated ubiquitination

(A) Validation of proteome screening by Western blotting with an anti-Prr7 antibody. $10 \mu \mathrm{g}$ of purified SM3 fractions from Nedd4-1/2 bDKO and Nedd4-1/2 bCtl mice were analyzed by Western blotting using an anti-Prr7 antibody (upper panel). Prr7 levels were upregulated in Nedd4-1/2 bDKO SM3 samples (EMX-Cre + ) compared to Nedd4-1/2 bCtl SM3 samples (EMX-Cre WT). Memcode-stained total proteins on nitrocellulose membrane were used as loading controls (lower panel). (B) The cytoplasmic tail of Prr7 is ubiquitinated by Nedd4-2. The myc-tagged cytoplasmic region of Prr7 (myc-Prr7 Cyt) was overexpressed in HEK293FT cells and immunopurified using anti-myc antibody coupled to agarose beads. Myc-Prr7 on the beads was used as a substrate for the in vitro ubiquitination assay. Note the depletion of parent bands of myc-Prr7 at the molecular weights of 35 and $37 \mathrm{kDa}$ and the shift of myc-Prr7 Cyt to the high molecular weight range (Ubiquitinated Myc-Prr7 Cyt) only in the presence of both E2 and Nedd4-2 (fourth lane). (C and D) In vivo ubiquitination of Prr7 by Nedd4-1 and Nedd4-2 E3 ligases. C-terminally FLAG-tagged full-length Prr7 (Prr7-FLAG) was overexpressed with 
EGFP-Nedd4-1 or EGFP-Nedd4-2 in HEK293FT cells and immunoprecipitated using antiFLAG antibodies coupled to agarose beads. Ubiquitination levels of Prr7-FLAG were analyzed by Western blotting using an anti-pan-ubiquitin, (C) or anti-K48-linked or anti-K63-linked polyubiquitin antibodies. (C) Western blotting using an anti-pan-ubiquitin antibody shows increases in signals when EGFP-Nedd4-1 (first lane) or EGFP-Nedd4-2 (second lane) were coexpressed with Prr7-FLAG as compared to negative controls (third to fifth lanes). (D) Prr7 is conjugated with K63-linked polyubiquitin chains by Nedd4-1 and Nedd4-2. Immunoprecipitated samples from the in vivo ubiquitination assay in $(\mathrm{C})$ were analyzed by Western blotting using antibodies specific to K48- or K63-linked polyubiquitin chains. Note that the signals from $25 \mathrm{ng}$ of K48-linked (sixth lane in the upper panel) and K63-linked tetraubiquitins (seventh lane in the lower panel) were comparable. Nedd4-1- and Nedd4-2dependent increases in signals at the high molecular weight range were observed only when blotted with the anti-K63-linked polyubiquitin antibody (bottom panel) but not with the antiK48-linked polyubiquitin antibody (upper panel).

\subsubsection{Nedd4-2 is the main E3 ligase for Prr7}

Next, we tested Prr7 levels in cortical homogenate from Nedd4-1/2 bDKO and control mice to check if ubiquitination of Prr7 leads to lysosomal degradation. The cortical homogenates from Nedd4-1/2 bDKO and control mice were analyzed by Western blotting using an anti-Prr7 antibody. As shown in Figure 3-12A, Prr7 levels in cortical homogenate from 6 week-old Nedd4-1/2 bDKO mice was upregulated by $~ 2.2$-fold as compared to control (Nedd4-1/2 bCtl $1.00 \pm 0.08, \mathrm{~N}=3 ; \mathrm{Nedd} 4-1 / 2$ bDKO $2.18 \pm 0.13, \mathrm{~N}=3 ; \mathrm{p}=0.0014)$. Taken together with the data showing ubiquitination of Prr7 by Nedd4-2 with K63-linked polyubiquitin chains, this result indicates that ubiquitination of Prr7 by Nedd4-2 might lead to lysosomal degradation of Prr7. Given that Prr7 is a neuronal protein (Murata et al., 2005), we tested next whether the upregulation of Prr7 levels in the Nedd4-1/2 bDKO is due to the neuronal loss of Nedd4-1 and

Nedd4-2. Thus, we analyzed Prr7 levels in cortical homogenates from control and Nedd4-1/2 nDKO mice, which express Cre recombinase only in postmitotic neurons but not in glia, by Western blotting using an anti-Prr7 antibody. As expected, the level of Prr7 was upregulated in the Nedd4-1/2 nDKO as compared to control (Figure 3-12B: Nedd4-1/2 nCtl 1.00 $\pm 0.082, \mathrm{~N}=3$; Nedd4-1/2 nDKO 2.45 $\pm 0.085, \mathrm{~N}=3 ; \mathrm{p}=0.0003$ ), indicating that upregulation of Prr7 in Nedd4$1 / 2$ bDKO is mainly caused by the deletion of Nedd4-1 and Nedd4-2 in neurons. 



Figure 3-12. Prr7 levels in different Nedd4-1 and Nedd4-2 conditional knockout lines

Cortical homogenates from 6 weeks-old conditional knockout and control animals were subjected to Western blotting using anti-Prr7 (upper panels) and anti-tubulin antibodies (bottom panels). Relative Prr7 levels were quantified by normalizing the signals from the anti-Prr7 antibody to the signal from the anti-tubulin antibody. Data are represented as mean \pm SEM. (A) 
Levels of Prr7 were upregulated in Nedd4-1/2 bDKO cortical homogenates. Nedd4-1/2 bCtl, $1.00 \pm 0.078, \mathrm{~N}=3$; Nedd4-1/2 bDKO, $2.18 \pm 0.13, \mathrm{~N}=3 ; \mathrm{p}=0.0014$. (B) Prr7 levels were increased in Nedd4-1/2 nDKO cortical homogenates. Nedd4-1/2 nCtl $1.00 \pm 0.082, \mathrm{~N}=3$; Nedd4-1/2 nDKO, $2.45 \pm 0.085, \mathrm{~N}=3$; $\mathrm{p}=0.0003$. (C) Prr7 levels were upregulated in cortical homogenates from Nedd4-2 bKO. Nedd4-2 bCtl $1.000 \pm 0.127, \mathrm{~N}=3$; Nedd4-2 bKO, $1.94 \pm$ $0.072, \mathrm{~N}=3 ; \mathrm{p}=0.0030$. (D) Levels of Prr7 were not changed in Nedd4-1 bKO cortical homogenates. Nedd4-1 bCtl, $1.000 \pm 0.122 \mathrm{~N}=4$; Nedd4-1 bKO, $0.77 \pm 0.102, \mathrm{~N}=4 ; \mathrm{p}=0.2011$.

Our data on the ubiquitination of Prr7 in vivo and in vitro showed that Nedd4-2 has a stronger intrinsic activity to conjugate K63-linked polyubiquitin chains to Prr7 than Nedd4-1. Thus, we analyzed the levels of Prr7 in cortical homogenates from Nedd4-1 bDKO, Nedd4-2 bDKO and control mice. As shown in Figure 3-12C, the levels of Prr7 were upregulated by 2fold in cortical homogenates from Nedd4-2 bKO as compared to control (Nedd4-2 bCtl $1.00 \pm$ $0.13, \mathrm{~N}=3$; Nedd4-2 bKO $1.94 \pm 0.071, \mathrm{~N}=3 ; \mathrm{p}=0.003)$. However, the Prr7 level was not altered in Nedd4-1 bKO as compared to control (Figure 3-12D: Nedd4-1 bCtl 1.00 $\pm 0.12, \mathrm{~N}=4$; Nedd4$1 \mathrm{bKO} 0.77 \pm 0.10, \mathrm{~N}=4 ; \mathrm{p}=0.2011)$. Altogether, our results indicate that Nedd4-2 is the main E3 ligase for Prr7 in neurons.

\subsection{Role of WWP family $\mathbf{E 3}$ ligases in neuron development}

\subsubsection{WWP family E3 ligases play an important role in dendrite branching}

\subsubsection{VCP and CAPZA1 were identified as substrates of WWP1 and WWP2 with}

\section{proteome screening for less ubiquitinated proteins in the WWP1;WWP2 double}

\section{KO mouse brain}

In order to identify substrates of WWP1 and WWP2 in mouse brain, we applied Ubiscan, a service provided by Cell Signaling Technology (PTMscan) for WWP1 and WWP2 mutant mice. Briefly, brain homogenates from $W W P 1^{\mathrm{f} / \mathrm{f}} ; W W P 2^{\mathrm{f} / \mathrm{f}} ; E M X 1-\mathrm{Cre}^{+/-}(\mathrm{WWP} 1 / 2$ $\mathrm{bDKO})$ and $W W P 1^{\mathrm{f} / \mathrm{f}} ; W W P 2^{\mathrm{f} / \mathrm{f}} ; E M X 1-\mathrm{Cre}^{\mathrm{WT}}(\mathrm{WWP} 1 / 2 \mathrm{bCtl})$ were prepared in the presence of NEM to inactivate de-ubiquitinase enzymes. Proteins were digested with trypsin, yielding peptides with diglycine remnants from ubiquitin conjugated on lysine residues (K- $\varepsilon-G G)$ (Figures 3-13A and 3-13B). Peptides conjugated with diglycine remnants were purified from trypsin-treated cortical lysates with anti-K- $\varepsilon-\mathrm{GG}$ antibodies coupled to agarose beads (Cell Signaling). Peptides immunoprecipitated from WWP1/2 bDKO and WWP1/2 bCtl were subjected to label-free mass spectrometric analysis to compare levels of peptides in the two genotypes. Several peptides showed reduced signals in mass spectropmetry in WWP1/2 bDKO 
brain homogenates as compared to WWP1/2 bCtl brain homogenates. Proteins containing such peptides were considered as putative candidate substrates of WWP1 and WWP2 (Figure 3-13C).

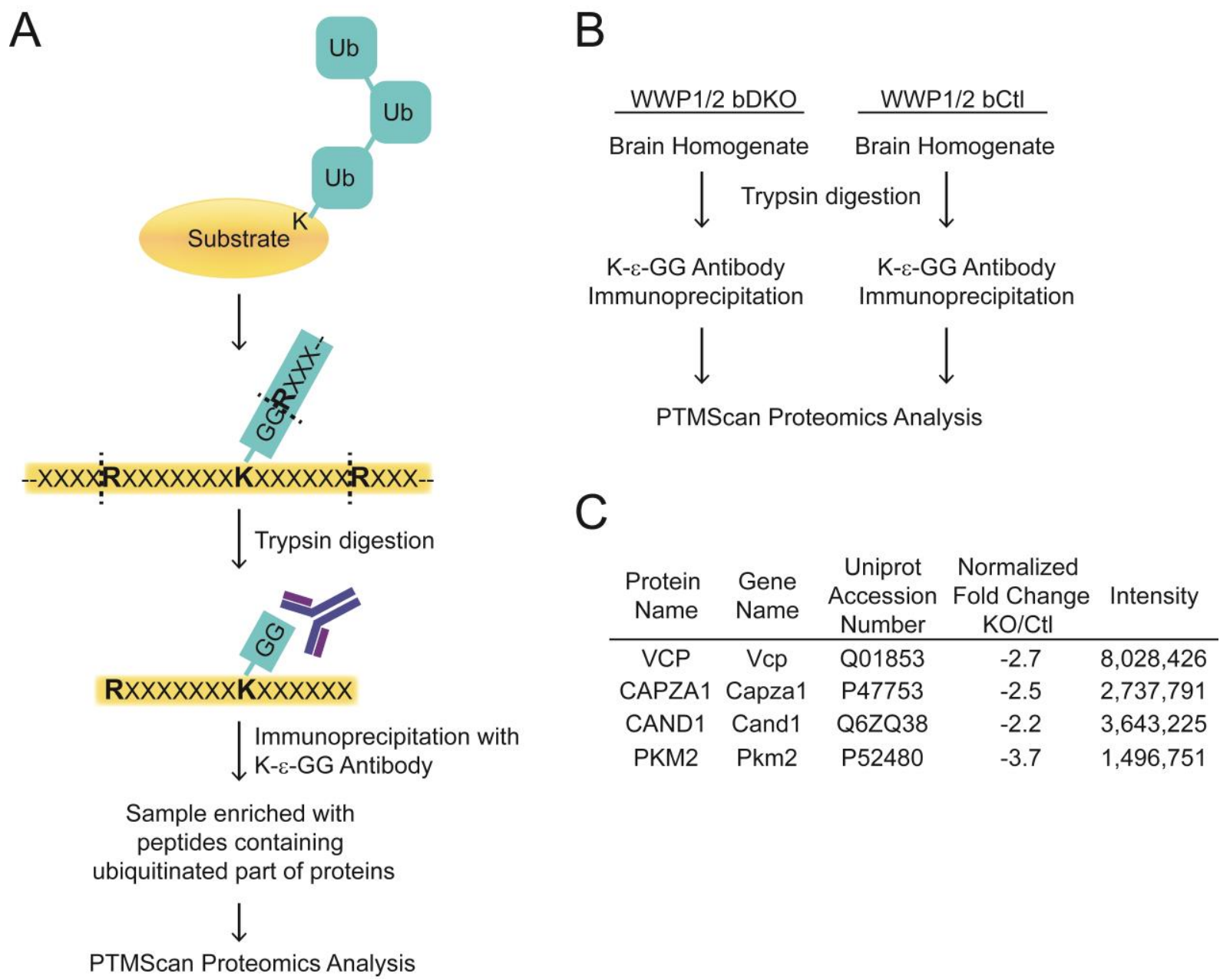

Figure 3-13. Screening of substrates of WWP1 and WWP2 by quantitative mass spectrometry

(A) Immunopurification of ubiquitinated peptides from brain homogenates for analysis by quantitative mass spectrometry. Trypsin digests the peptide bond between the arginine residue and diglycine remmants in ubiquitin, resulting in an attachment of diglycine remnants to


immunopurified from cerebral homogenates using an antibody specific for K- $\varepsilon$-GG. (B) Brain homogenates prepared from WWP $1 / 2 \mathrm{bDKO}$ and WWP $1 / 2 \mathrm{bCtl}$ mice were subjected to trypsin digestion and purification of K- $\varepsilon$-GG containing peptides. Quantitative mass spectroscopy was performed to compare the levels of K-E-GG containing peptides in WWP1/2 bDKO and WWP1/2 bCtl samples. (C) List of potential substrate proteins of WWP1 and WWP2 identified in Ubi-scan. Proteins including peptides with less abundance in WWP1/2 bDKO than in WWP1/2 are listed as potential substrates of WWP1 and WWP2. 
Next, we validated the results of Ub-scan. Candidate proteins, such as F-actin-capping protein subunit alpha-1 (CAPZA1), pyruvate kinase M2 (PKM2), and Cullin-associated NEDD8-dissociated protein 1 (CAND1), were overexpressed as Myc- or V5-tagged recombinant proteins in HEK293FT cells and immunoprecipitated using anti-myc or anti-V5 antibodies coupled to agarose beads and used as substrates in in vitro ubiquitination assay. Valosin-containing protein (VCP), another candidate protein, was expressed as a His6-tagged protein in E.coli and purified using nickel agarose beads. Subsequently, purified substrate proteins were incubated with recombinant ubiquitin, E1, E2 and E3 enzymes (WWP1, WWP2, or Itch) in the presence of ATP, and reactions were analyzed by Western blotting using antibodies against the individual tags. Of all candidate proteins tested, VCP, Capza1, Pkm2 were ubiquitinated in vitro by WWP E3 ligases, while CAND1 was not (Figures 3-14A-3-14D). These results indicate that VCP, CAPZA1, PKM2 are substrate proteins of WWP1 or WWP2 in the mouse brain.



Figure 3-14 Validation of the proteomic screening results

Candidate substrate proteins of WWP1 and WWP2 identified in the proteomic screening (Figure3-13C) were validated using an in vitro ubiquitination assay. Recombinant candidate proteins were either overexpressed in and immunopurified from HEK293FT cells (V5-CAND1, myc-PKM2, mycCAPZA1) or purified from E. coli (myc-VCP). (A) V5-CAND1 was not 
ubiquitinated by WWP1 or WWP2 in vitro. (B) myc-PKM2 was mono-ubiquitinated by WWP1. (C) myc-CAPZA1 was poly-ubiquitinated by WWP1. (D) VCP was mono-ubiquitinated or diubiquitinated by WWP1, WWP2 and Itch E3 ligases. Note the efficiency of VCP ubiquitination by WWP subfamily E3 ligases. More than $70 \%$ of the substrate were ubiquitinated.

\subsubsection{WWP1/2 double knockout neurons show enhanced neurite growth}

In order to study the role of WWP1 and WWP2 in dendrite development, we analyzed the morphology of cultured hippocampal neurons prepared from $W W P 1^{\mathrm{f} / \mathrm{f}} ; W W P 2^{\mathrm{f} / \mathrm{f}} ; N E X 1-\mathrm{Cre}^{+/-}$

$(\mathrm{WWP} 1 / 2 \mathrm{nDKO})$ and $W W P 1^{\mathrm{f} / \mathrm{f}} ; W W P 2^{\mathrm{f} / \mathrm{f}} ; N E X 1-\mathrm{Cre}^{\mathrm{WT}}(\mathrm{WWP} 1 / 2 \mathrm{nCtl})$ mice. Neurons were transfected with a GFP expression vector using the calcium phosphate method at DIV1 and fixed in order to study neurite branching at DIV7. The complexity of neurites was analyzed on binarized images of the GFP signal from individual neurons using Sholl analysis. In Sholl analysis, concentric circles with $10 \mu \mathrm{m}$ intervals are drawn around the cell soma and the numbers of neurite intersections on each circle are counted (Kawabe et al., 2010; Sholl, 1953). In contrast to the neurons from most KOs or KDs of E3 ligase genes/mRNAs (e.g. cdc20 KD, Nedd4-1 KO, Fbxo31 KD), WWP1/2 nDKO hippocampal neurons show enhanced neurite branching as compared to WWP1/2 nCtl neurons (Figures 3-15A-C), with an increased number of total intersections with Sholl circles (Figure 3-15D: WWP1/2 nCtl 48.21 \pm 2.62 , n=38; WWP1 nDKO $71.90 \pm 2.86, \mathrm{n}=42, \mathrm{p}<0.0001)$. Thus, morphological analysis of DIV7 hippocampal neurons shows that WWP1 and WWP2 impair neurite development. 
A
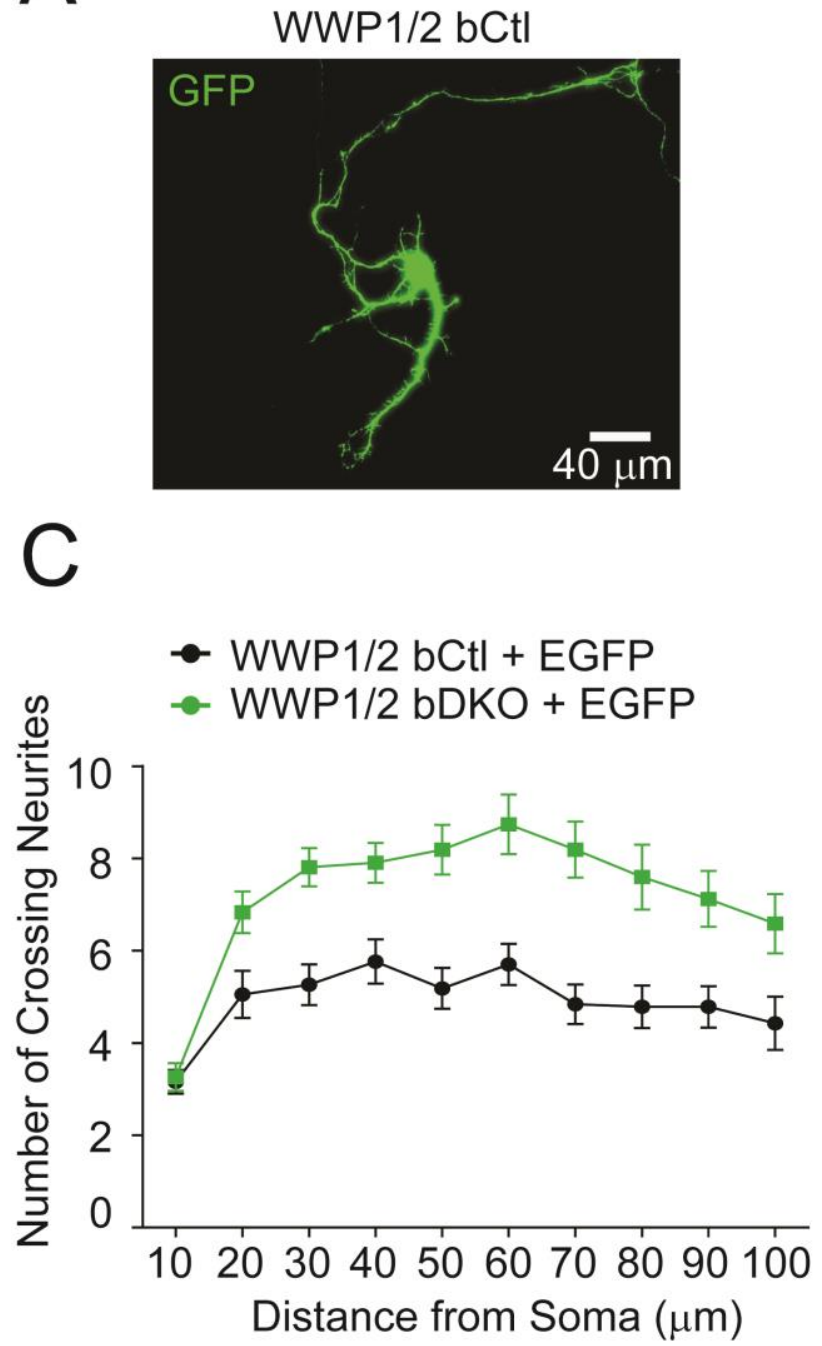

B


Figure 3-15. WWP1/2 DKO neurons show enhanced neurite branching

(A and B) Representative images of primary hippocampal neurons prepared from WWP1/2 bCtl (A) and WWP1/2 DKO (B) mice. Neurons were transfected with an EGFP expression vector at DIV1 and fixed at DIV7. Scale bars, $20 \mu \mathrm{m}$. (C) Sholl analyses of WWP1/2 bCtl and WWP1/2 bDKO neurons. Results are represented as means \pm SEM. WWP1/2 bCtl n=38, WWP1/2 bDKO $\mathrm{n}=42$ neurons. (D) Averaged total number of intersections of neurites and Sholl circles per neuron. Results are represented as means \pm SEM. WWP1/2 nCtl 48.21 \pm 2.62 , n=38; WWP1 nDKO $71.90 \pm 2.86, \mathrm{n}=42, \mathrm{p}<0.0001$. 


\subsubsection{CAPZA1 overexpression in wild-type hippocampal neurons phenocopies the dendrite branching effect of WWP1/2 nDKO}

One of the main molecular determinants of neurite development is the regulation of the actin cytoskeleton. Capping proteins have a crucial role for the dynamics of actin cytoskeleton organization (Schafer and Cooper, 1995; da Silva and Dotti, 2002). In order to study if CAPZA1, one of the substrates of WWP1 (Figure 3-13 and 3-14), is involved in the regulation of dendrite branching, we overexpressed N-terminally myc-tagged CAPZA1 in hippocampal neurons. Wild-type hippocampal neurons were transfected with a GFP expressing vector alone or with vectors expressing GFP and myc-CAPZA1. Hippocampal neurons were fixed at DIV7 and subjected to Sholl analysis using binarized images of the GFP signal. As shown in Figure 3-16, hippocampal neurons overexpressing myc-CAPZA1 showed enhanced neurite branching as compared to control neurons, with an increased total number of intersections (Control $55.97 \pm$ $3.233, \mathrm{n}=32 ;$ myc-CAPZA1 $73.33 \pm 3.58, \mathrm{n}=40 ; \mathrm{p}=0.0008$ ). This phenotypic change resembles that of WWP1/2 nDKO neurons, indicating that the functional upregulation of CAPZA1 could be the cause of the enhanced branching of neurites in WWP1/2 nDKO neurons. 


\section{A}
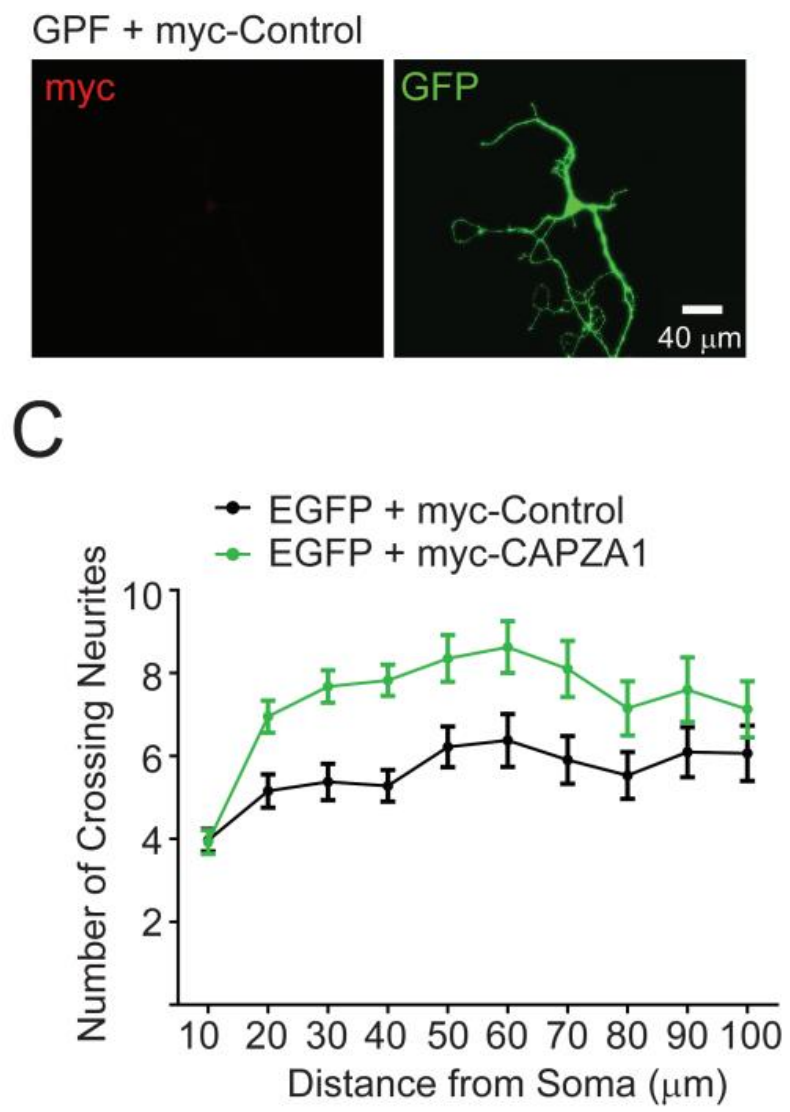

GPF + myc-CAPZA1
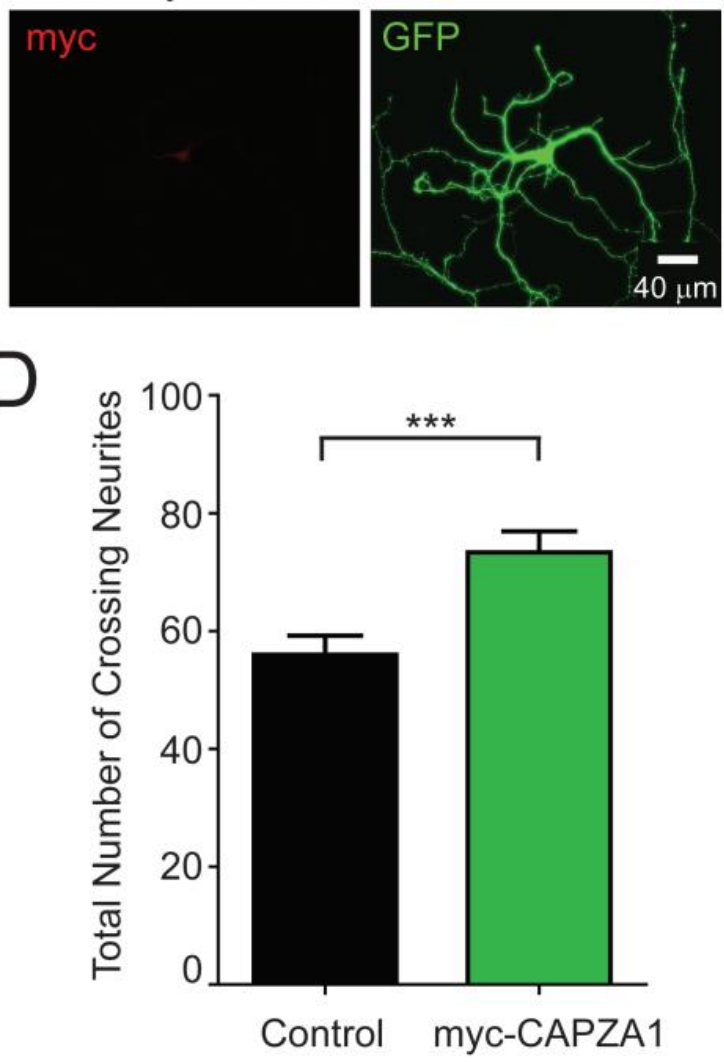

Figure 3-16. myc-CAPZA1 overexpression leads to enhanced neurite branching

(A and B) Representative images of wild-type primary hippocampal neurons at DIV7. Neurons were transfected with EGPF expression vector alone (A) or EGFP and myc-CAPZA1 expression vectors (B) at DIV1 and fixed at DIV7. Scale bars, $20 \mu \mathrm{m}$. (C) Sholl analysis of wild-type neurons overexpressing EGFP alone or EGFP and myc-CAPZA1. Results are represented as means \pm SEM. Control $n=32$, myc-CAPZA1 $n=40$ neurons. (D) Averaged total number of intersections of neurites and Sholl circles per neuron. Results are represented as means \pm SEM. Note that overexpression of CAPZA1 in wild type neurons has almost the same effect as KO of WWP1 and WWP2 (compare Figures 3-15C and 3-15D with Figures 3-16C and 3-16D). Control $55.97 \pm 3.233, \mathrm{n}=32 ;$ myc-CAPZA1 $73.33 \pm 3.58, \mathrm{n}=40 ; \mathrm{p}=0.0008$. 


\section{DISCUSSION}

\subsection{Function of the Nedd4 subfamily E3 ligases in mouse brain}

\subsubsection{Novel candidate proteins as substrates of Nedd4-1 or Nedd4-2 are identified in a physiological substrate screening in mouse brain}

There have been a number of biochemical and proteomics studies to identify putative substrates of Nedd4-1 and Nedd4-2 in vitro. For instance, Nedd4-1 was purified and identified as an E3 ligase for phosphatase and tensin homolog (PTEN) in HeLa cell lysates (Wang et al., 2007). Even though Nedd4-1 has been reported to regulate the expression level, phosphatase activity, and localization of PTEN (Christie et al., 2012; Trotman et al., 2007), subsequent studies have produced different results, indicating that the protein level and localization of PTEN is not changed in Nedd4-1 knockout and knockdown cells (Cao et al., 2008; Fouladkou et al., 2008). In addition, several studies have proposed different E3 ligases, including WWP2, XIAP, CHIP to regulate PTEN (Ahmed et al., 2012; Maddika et al., 2011; Van Themsche et al., 2009). In a recent study, it has been reported that PTEN is not a downstream target of Nedd4-1 but an upstream regulator of Nedd4-1, acting by suppressing its translation through the MTORC1-PI3K pathway (Hsia et al., 2014). Therefore, in vitro biochemical assays and proteomics approaches are not always sufficient to reliable identify substrate proteins for E3 ligases.

In the present study, we tackled this issue by using the brain specific Nedd4-1; Nedd4-2 double conditional knockout mice line, which is probably one of the most stringent tools available to identify substrates of these proteins. Given the fact that Nedd4-1 and Nedd4-2 localize to synapses and conjugate K63-linked polyubiquitin chains, which have been shown to regulate the endocytosis of substrate proteins (Kawabe et al., 2010; Maspero et al., 2013; Scudder et al., 2014), we analyzed synaptic membrane fractions from brain tissues, which enabled us to avoid cell type specific and context dependent artifacts in substrate identification. Our data show that with this method we can also detect proteins on the perisynaptic end-feet of astrocytes at tripartite synapses. Out of five putative substrate proteins identified to be upregulated by iTRAQ mass spectrometry, we focused on two astrocytic proteins, Kir4.1 and Connexin-43, and one postsynaptic neuronal protein, Prr7. The other two candidate proteins identified were mitochondrial proteins, NADH dehydrogenase iron-sulfur protein 6 (Ndufs6) and CDGSH iron-sulfur domain containing protein 1 (Cisd1), and not followed-up. 
Identification of mitochondrial proteins in SM3 fractions by proteomic screening might be due to inevitable slight contaminations of sample by synaptic mitochondria. However, purified SM3 fractions were highly depleted of synaptic cytoplasmic content (Figure 3-2), and most of the peptides identified by mass spectrometry were transmembrane or membrane anchored proteins. Although we did not test Ndufs6 and Cisd1 in our study, Nedd4-1 or Nedd4-2 might specifically regulate these mitochondrial proteins since other mitochondrial proteins were not upregulated in Nedd4-1/2 bDKO SM3 fractions. The Western blotting-based validation of the upregulated levels of Kir4.1, Connexin-43 and Prr7, along with the finding that all these proteins are ubiquitinated by Nedd4-1 and Nedd4-2 (Figure 3-3, 3-5, 3-11), show that all three proteins we tested are prominent substrates of Nedd4-1 or Nedd4-2 E3 ligases. This shows that our approach of combining iTRAQ quantitative mass spectrometry with synaptic membrane purification in order to identify putative substrate proteins of E3 ligases is highly reliable. The high accuracy in the identification of substrate proteins in our study is due to several critical steps during the analysis. Firstly, the purification of SM3 fractions provides a rather low complexity sample for mass spectrometry, which enabled us to obtain more reliable and accurate data in the quantitative analysis. Secondly, the automated in-gel digestion and iTRAQ labelling methods minimize the variability in protein digestion and labelling efficiency. Gel-based labelling methods also had less complexity of samples for mass spectrometry analyses since protein bands are separated before trypsin digestion and iTRAQ labelling (Schmidt et al., 2013). Thirdly, we limited the identification to substrates that were identified by at least five peptides in order to dilute signal artifacts due to the biased labelling efficiencies of different iTRAQ labels. Lastly, we performed complementary experiments in mass spectrometric analysis in term of labels used for control and knockout SM3 fractions.

\subsubsection{Astrocytic Nedd4-1 and Nedd4-2 are important for the regulation of Kir4.1 and Connexin-43}

Nedd4-1 and Nedd4-2 were first identified in a screen to identify developmentally downregulated genes in mouse brain (Kumar et al., 1992). Expression of Nedd4-1 and Nedd42 is not only regulated in a development-dependent manner but also spatially, so that Nedd4-1 is expressed ubiquitously in several tissues such as brain, muscle, liver and kidney while Nedd42 expression seems to be more restricted in brain, liver and kidney (Anan et al., 1998; Donovan 
and Poronnik, 2013; Kumar et al., 1997). In a number of studies, Nedd4-1 and Nedd4-2 were proposed to be involved in neurite outgrowth, neuronal cell fate determination, and neuronal cell survival. In the context of neurite outgrowth, it has been reported that Nedd4-1 conjugates mono-ubiquitination to GTP-bound Rap2A, which abolishes the Rap2A interaction with TRAF2 and NCK-interacting protein kinase (TNIK), so that Nedd4-1 acts as a positive regulator of dendrite growth (Kawabe et al., 2010). In addition to the function of Nedd4-1 in dendrite development, recent functional analyses of Nedd4-1 and Nedd4-2 double conditional knockout mice have revealed that Nedd4-1 and Nedd4-2 are also involved in axon growth in hippocampal neurons (Hsia et al., 2014). In terms of neuronal cell fate, Nedd4-1 has been reported to regulate dorsoventral patterning of the neuronal ectoderm in zebrafish by regulating $\Delta \mathrm{Np} 63 \alpha$ (Bakkers et al., 2005). In Drosophila, Nedd4-1 conjugates polyubiquitin chains to Notch and leads to its endocytosis, indicating that a regulatory function of Nedd4 in cell fate determination is conserved in Drosophila (Dalton et al., 2011; Sakata et al., 2004). In terms of neuronal cell survival, it has been reported that Nedd4-2 facilitates the ubiquitination of TrkA, leading to its trafficking to late endosomes upon NGF treatment in cultured dorsal root ganglion neurons (Yu et al., 2011, 2014).

While there has been significant interest in the functions of Nedd4-1 and Nedd4-2 in the developing brain, the functions of Nedd4-1 and Nedd4-2 in adult brain remain uncharted territory. Several biochemical studies have proposed that voltage-gated sodium channels $\left(\mathrm{Na}_{\mathrm{v}} 1.2, \mathrm{Na}_{\mathrm{v}} 1.3, \mathrm{Na}_{\mathrm{v}} 1.5\right.$, and $\mathrm{Na}_{\mathrm{v}} 1.7$ ), voltage-gated potassium channels (KCQN2/3 and KCQN3/5), and voltage-dependent calcium channels $\left(\mathrm{Ca}_{\mathrm{v}} 1.2\right)$ are regulated by Nedd4 subfamily members, indicating possible roles of neuronal Nedd4-1 and Nedd4-2 in the regulations of neuronal excitability (Ekberg et al., 2007; Fotia et al., 2004; Rougier et al., 2005, 2011; Schuetz et al., 2008). In our study, we identified two novel substrate proteins for Nedd4-1 and Nedd4-2, Kir4.1, an inwardly rectifier channel expressed mainly in astrocytes and Connexin-43 one of the main gap junction proteins in astrocytes.

\subsubsection{Kir4.1 is a substrate of Nedd4 subfamily E3 ligases in astrocytes}

Kir4.1 is the main potassium channel in astrocytic end-feet at synapses (Robel and Sontheimer, 2015). Propagation of action potentials upon neuronal activity results in increases of the extracellular potassium concentration (Nicholson and Syková, 1998). During 
hyperactivity, extracellular potassium concentrations have been reported to elevate from $3 \mathrm{mM}$ to 10-12 mM (Heinemann and Lux, 1977). Considering that an increase in extracellular potassium leads to more positive membrane potentials, this affecting the activation of ion channels, transporters, and receptors, the clearance of excess potassium from extracellular space at axons and synapses strongly affects neuronal excitability (Seifert and Steinhäuser, 2013). It has been reported that brain-specific Kir4.1 conditional knockout mice show pronounced behavioral changes with ataxia and seizures (Djukic et al., 2007). In whole-genome linkage studies, homozygous missense mutations in KCNJ10 gene which encodes the Kir4.1 channel, were been reported as the cause of SeSAME syndrome (EAST Syndrome), which is characterized by seizures, ataxia, sensorineural deafness, mental retardation, and electrolyte imbalance (Bockenhauer et al., 2009; Scholl et al., 2009). Further, an impaired function of Kir channels has been reported in the CA1 region of sclerotic human epileptic hippocampal specimens (Kivi et al., 2000). Our data indicate that Nedd4-1 and Nedd4-2 conjugate K63-linked polyubiquitin chains to the C-terminal cytoplasmic tail of Kir4.1 and thus regulate Kir4.1 protein level in astrocytes (Figure 3-4 and 3-5), and thereby might play an important role in the regulation of neuronal excitability in adult brain through astrocytes.

In addition to potassium buffering, astrocytes play an important role in the clearance of glutamate from the synaptic cleft (Anderson and Swanson, 2000). Indeed, glutamate uptake and potassium buffering are interdependent processes. Astrocytic Kir4.1 plays an important role in establishing the negative membrane potential, providing the driving force of glutamate uptake by astrocytes (Wetherington et al., 2008). Correspondingly, downregulation or selective deletion of Kir4.1 in cultured astrocytes lead to inhibition of glutamate uptake by astrocytes (Djukic et al., 2007; Kucheryavykh et al., 2007). After uptake into astrocytes, glutamate is converted to glutamine by glutamine synthetase, and glutamine cycles back to the neurons through a process called glutamate-glutamine cycle (Danbolt, 2001). In neurons, glutamine can then be converted into glutamate or gamma-aminobutiric acid (GABA) (Robel and Sontheimer, 2015). Disruption of the glutamate-glutamine cycle by selective inhibitors of neuronal glutamine transporters or astrocytic glutamine synthetase significantly reduced evoked inhibitory postsynaptic currents (eIPSCs) in hippocampal CA1 pyramidal neurons, indicating that glutamate uptake by astrocytes and the glutamate-glutamine cycle has an important role in inhibitory synaptic transmission in the brain (Liang et al., 2006), while excitatory synaptic transmission can work 
independently from the glutamate-glutamine cycle (Kam and Nicoll, 2007). Therefore, imbalanced glutamate uptake by astrocytes might affect the excitation/inhibition balance in neuronal networks (Robel and Sontheimer, 2015). Given that Nedd4-1 and Nedd4-2 regulate Kir4.1 levels in astrocytes, they might have a role in neuronal networks in terms of excitation/inhibition balance through astrocytes. This seems to be the case and roles of Nedd42 in neuronal excitability and excitation/inhibition balance in neuronal networks will be further discussed in section 4.1.5.

\subsubsection{Nedd4-2 is the dominant E3 ligase regulating Connexin-43 in astrocytes}

Nedd4-1 was identified as an E3 ligase for Connexin-43 by affinity purification using GST-tagged Connexin43 from a hepatocyte cell line (Leykauf et al., 2006). In the same report, the second WW domain of Nedd4-1 was identified as the Connexin-43-binding domain, and the PPXY motif in Connexin-43 was mapped as the Nedd4-1-binding site. Strikingly, knockdown of Nedd4-1 by siRNA was shown to cause an increase in membrane localization of Connexin43 in a cultured hepatocyte cell line (Leykauf et al., 2006), and a follow-up study proposed that Nedd4-1 conjugates multiple single ubiquitin moieties to Connexin- 43 and that ubiquitinated Connexin-43 interacts with ubiquitin interacting motif (UIM) of Eps15, leading the internalization of Connexin-43 (Girão et al., 2009).

As mentioned already, such knockdown studies using cultured cell lines are often problematic. Although we identified Connexin-43 as a protein that is upregulated in the Nedd4$1 / 2 \mathrm{bDKO}$ as compared to Nedd4-1/2 bCtl in our proteome screening (Figure 3-5A), quantitative Western blotting in Figure 3-6 demonstrated that the Connexin-43 level is upregulated in the Nedd4-2 bKO but not in the Nedd4-1 bKO, indicating that Nedd4-2 is the dominant E3 ligase for Connexin-43 in the brain. Supporting this notion, the results of the in vivo ubiquitination assays demonstrated that Nedd4-2 has a stronger activity to conjugate K63-linked polyubiquitin chains to Connexin-43 than Nedd4-1 (Figure 3-5B-C). However, different degrees of upregulation of Connexin-43 levels in Nedd4-1/2 bDKO (1.95 fold) and Nedd4-2 bKO (1.55 fold) indicate that in the absence of Nedd4-2, Nedd4-1 might partially compensate the loss of function of Nedd4-2 (Figure 3-6A, 3-6C).

Astrocytes are coupled with each other through gap junctions and thereby form a large intercellular network in the brain that allows astrocytes to disperse ions and small molecules, 
such as ATP, $\mathrm{K}^{+}, \mathrm{Ca}^{2+}$, glutamate, and cAMP (Dbouk et al., 2009; Wetherington et al., 2008). Connexin-43 is one of the main gap junction proteins expressed in astrocytes. Dye-coupling experiments revealed that in Connexin-43 conditional knockout mice, the overall astrocytic intercellular networking is reduced by 50\% (Theis et al., 2003a, 2003b). In a study with Connexin-30 and Connexin-43 double knockout mice, it was shown that astrocyte coupling through these connexins accelerates potassium buffering in hippocampal slices and that mice lacking Connexin-30 and Connexin-43 are more susceptible to epileptiform events (Wallraff, 2006). Astrocytic gap junction coupling is also important for ICWs in the astrocytic network and gap junction inhibitors reduce or block ICWs (Rouach and Giaume, 2001; Venance et al., 1995). In addition to gap junction coupling between neighboring astrocytes, one of the molecular mechanisms in the propagation of ICWs is the release of ATP through Connexin-43 hemichannels (Stout et al., 2002). Purinergic receptors are involved in ICWs by triggering the $\mathrm{IP}_{3}$-signaling pathway (Suadicani et al., 2004; Venance et al., 1997). Given that Nedd4-2 regulates the level of Connexin-43 in astrocytes, our data indicate that Nedd4-2 might play an important role in the regulation of ICWs in astrocytic networks, eventually affecting the function of neuronal networks.

Regulation of Connexin-43 in astrocytic network might also play an important role in potassium buffering (Wallraff, 2006), suggesting dual effects of Nedd4-2 on potassium clearance in the brain through both Kir4.1 and Connexin-43 in astrocytes.

\subsubsection{Nedd4-1 and Nedd4-2 in astrocytes regulate neuronal network function through Kir4.1 and Connexin-43}

Considering two critical roles of Nedd4-1- and Nedd4-2-dependent regulation of Kir4.1 and Connexin-43 in astrocytes, spatial potassium buffering and ICWs, we investigated neuronal functions in our knockout mouse lines. Our data on gamma oscillations (Figure 3-8E, 3-9E, 3$10 \mathrm{E})$ indicate that the synchronization of neuronal function is impaired in the CA3 pyramidal region of the hippocampus in Nedd4-1/2 bDKO and Nedd4-2 bKO, but not in Nedd4-1/2 nDKO mice. This shows that glial Nedd4-1 and Nedd4-2 have an impact on neuronal function in the hippocampus. Imbalanced potassium clearance because of the increased level of Kir4.1 might disrupt the neuronal function in Nedd4-1/2 bDKO and Nedd4-2 bKO mice. The increased level of Kir4.1 might also affect the glutamate uptake by astrocytes leading to alterations in the 
excitation/inhibition balance due to an imbalance in the glutamate-glutamine cycle. Impairments in gamma oscillations in Nedd4-1/2 bDKO and Nedd4-2 bKO might be due to alterations in excitation/inhibition balance in the neuronal network as it has been reported that the excitation/inhibition balance in the brain is important for proper neuronal network function (Mann and Mody, 2009; Snijders et al., 2013). Similarly, altered hippocampal synaptic inhibition in Neuroligin-4 knockout mice results in reduced gamma oscillation in hippocampal slices (Hammer et al., 2015), and kainate-induced gamma oscillations are highly dependent on synaptic inhibition by parvalbumin-expressing basket cells (Bartos et al., 2007).

Another reason of reduced gamma oscillatory activity in Nedd4-1/2 bDKO and Nedd42 bKO might be the increased levels of Connexin-43 in astrocytes. It has been reported, for instance, that Connexin-43 overexpression alters the properties of ICWs (Suadicani et al., 2004) and astrocytic intracellular calcium responses precede carbachol-induced gamma oscilaltions in the CA3 region of the hippocampus (Lee et al., 2014). Thus, the increased levels of Connexin43 in Nedd4-1/2 bDKO and Nedd4-2 bKO mice might perturb the properties of ICWs, thereby causing reduced gamma oscillatory activities in the CA3 region of the hippocampus. Additionally, the increased levels of Connexin-43 might result in high copy numbers of Connexin-43 hemichannels in Nedd4-1/2 bDKO and Nedd4-2 bKO mice, which might lead to an increased levels of ATP-release by hemichannels. The increased levels of ATP-release by astrocytes might lead to changed properties of ICWs through purinergic receptors on neighbor astrocytes, thereby leading to reduced gamma oscillatory activities in Nedd4-1/2 bDKO and Nedd4-2 bKO mice. Also, the increased levels of Connexin-43 in Nedd4-1/2 bDKO and Nedd4$2 \mathrm{bKO}$ mice, together with increased levels of Kir4.1, might affect the potassium buffering by astrocytes, which might lead to altered neuronal excitability, thereby affecting gamma oscillatory activities in the CA3 region of hippocampus. It would be particularly important to study if specific inhibitors of Kir4.1 and Connexin-43, such as fluoxetine hydrochloride and GAP-26 peptide respectively (Desplantez et al., 2012; Ohno et al., 2007), rescue the phenotypes of reduced gamma oscillatory activities in the CA3 region of the hippocampus in Nedd4-1/2 bDKO and Nedd4-2 bKO mice. This experiment would lead us to conclude direct impact of increased levels of Kir4.1 and/or Connexin-43 in Nedd4-1/2 bDKO and Nedd4-2 bKO mice on gamma oscillatory activities in CA3 region of hippocampus. It would be also particularly important to study if the increased levels of Kir4.1 and Connexin-43 have an impact on neuronal 
excitability in Nedd4-1/2 bDKO and Nedd4-2 bKO by measuring the mEPSCs and mIPSCs in pyramidal neurons at the CA3 region of hippocampus in brain slices. This experiment would lead us to conclude if activities of excitatory or inhibitory neurons are altered in the CA3 region of the hippocampus of Nedd4-1/2 bDKO and Nedd4-2 bKO mice.

\subsubsection{Regulation of Prr7 by Nedd4-2 might play a role in spine morphology}

Prr7 is a postsynaptic protein composed of a short extracellular N-terminal region, a single transmembrane domain, and a C-terminal cytoplasmic tail containing several PPXY motifs (Hrdinka et al., 2011). Prr7 interacts with PSD95 and NMDA receptor subunits NR1 and NR2B (Murata et al., 2005). Although the function of Prr7 is unknown, a recent proteomics screen of ubiquitinated synaptic proteins has revealed that Prr7 is one of the postsynaptic proteins that are highly ubiquitinated in the rat brain (Na et al., 2012).

In the present study, we discovered that Nedd4-2 conjugates K63-linked polyubiquitin chains to Prr7 and thereby regulates the levels of Prr7 (Figure 3-11). Considering that Prr7 interacts with NMDA receptor subunits, Nedd4-2 might regulate the function or localization of NMDA receptors through Prr7 ubiquitination. Analyses of dendritic spines on CA1 pyramidal cells of $N e d d 4-2^{\mathrm{f} / \mathrm{f}}-N E X-\mathrm{Cre}^{+/-}(\mathrm{Nedd} 4-2 \mathrm{nKO})$ and control mice have shown that in Nedd4-2 nKOs, the length of filipodia and mushroom spines are increased whereas spine density is not altered; additionally, the abundance of bifurcated spines is increased at the expense of mushroom spines (unpublished data from our group by Dr. Mateusz Cyryl Ambrożkiewicz). These findings and the produced data on Prr7 indicate that Nedd4-2 might play a role in dendritic spine development by regulating Prr7. It would be particularly important to study if the knockdown of Prr7 rescues the phenotype of spine morphology in Nedd4-2 nKO mice. This experiment would lead us to conclude the direct impact of increased levels of Prr7 on spine development.

\subsection{Function of WWP1 and WWP2 in the brain}

\subsubsection{WWP1 and WWP2 are negative regulators of neurite branching}

There have been several studies on the functions of WWP1 and WWP2 in dividing cells, including cartilage cells and transformed cell lines. However, their functions in postmitotic neurons are unknown. In the present study, we identified several putative substrate proteins for WWP1 and WWP2 using mouse brain tissue. The results of the PTMScan show that the 
ubiquitination levels of VCP, CAPZA1, PKM2, and CAND1 proteins are decreased in WWP1/2 bDKO brain homogenates. As we show in Figure 3-14, most of the candidates identified in the proteome screening were indeed ubiquitinated by WWP1 or WWP2 in vitro, indicating the reliability of this screening method.

We demonstrate that deletion of WWP1 and WWP2 leads to enhanced neurite development, indicating that WWP1 and WWP2 act as negative regulator of neurite growth (Figure 3-15). It is rare that deletion of a certain gene causes an augmentation of neurite arborization, although there are numerous examples of loss-of-function mutants causing an impairment of dendrite growth (Kawabe et al., 2010; Litterman et al., 2011; Park et al., 2015). In the present study, we investigated possible downstream targets of WWP1 and WWP2 in the regulation of dendrite development. Among the substrate proteins we identified, we first focused on CAPZA1. It is accepted widely that neurite development is dependent on the reorganization of actin cytoskeleton, which is mediated by different types of actin binding proteins, such as monomer binders, branch formation proteins, or capping proteins (Winder et al., 2005). Monomer binders interact with actin monomers and regulate their availability for actin filament assembly. One of the well-characterized monomer binders is profilin, which restricts actin monomer addition to barbed ends and prevents spontaneous nucleation (Nicholson-Dykstra et al., 2005). Another well-studied monomer binder protein is thymosin- $\beta 4$, which prevents ATPbound actin incorporation into both barbed and pointed ends (Hertzog et al., 2004; Irobi et al., 2004).

As for branch formation proteins, they regulate dendritic branching of actin filaments (Winder et al., 2005). The Arp2/3 complex, composed of Arp2 and Arp3 proteins is a wellknown branch formation complex (Svitkina and Borisy, 1999). Arp2 and Arp3 have a similar structure as actin and form a complex on the side of an existing actin filament to nucleate another filament by mimicking a barbed end (Pollard et al., 2003).

Regarding capping proteins, an important capping protein complex consists of a heterodimer composed of CAPZA and CAPZB, each of which has a molecular weight of $~ 30$ kDa. Mammalian genomes encode two CAPZA genes, CAPZA1 and CAPZA2. Capping proteins bind to barbed-ends of actin filaments and prevent filament elongation at these points. However this facilitates Arp2/3-mediated nucleation and formation of branches of F-actin (Akin and Mullins, 2008; Fan et al., 2011; Scott and Luo, 2001). Although the primary structures of 
CAPZA and CAPZB are not homologous, CAPZA and CAPZB share a similar secondary structure with a C-terminal $\square$-helix that binds to the barbed end of F-actin directly (Wear et al., 2003). Interestingly, our PTMScan mapped the potential lysine residue ubiquitinated by WWP1 and WWP2 at the C-terminal $\square$-helix of CAPZA1 and CAPZA2, indicating that ubiquitinated CAPZA might be deficient in binding to F-actin. Based on the hypothesis that loss of WWP1 and WWP2 therefore leads to a gain of CAPZA function, we overexpressed CAPZA1 in wild type primary cultured hippocampal neurons (Figure 3-16). Consistent with a previous report (Davis et al., 2009), overexpression of CAPZA1 enhanced dendrite branching and extension. However, while depletion of CAPZB results in impaired dendrite branching, recombinant CAPZB lacking the actin-binding region rescues the phenotype of CAPZB-knockdown neurons efficiently. Interestingly, CAPZB binds to tubulin directly and a tubulin-binding deficient mutant of CAPZB fails to rescue the knockdown neuron, indicating that tubulin-binding but not actin-binding activity of CAPZB is critical for dendrite development (Davis et al., 2009). One interesting model would be that tubulin-binding CAPZB forms a complex with actin-binding CAPZA and thus bridges microtubules and F-actin at the growth cone as, EB1 does (Tang et al., 2016). Indeed, CAPZA has a higher affinity to F-actin than CAPZB (Wear et al., 2003). It would be particularly important to study if overexpression of ubiquitination deficient and actin-binding deficient mutants of CAPZA results in higher and lower degrees of enhancement of dendrite branching, respectively. This experiment would lead us to conclude the impact of ubiquitination of CAPZA by WWP1 and WWP2 on dendrite development.

\subsubsection{Ubiquitination of VCP by WWP1 and WWP2 might play a role in dendritic spine formation}

In the PTMScan analysis, one of the candidates of substrates of WWP1 and WWP2 was VCP. VCP is an abundantly expressed ATP-driven chaperon protein composed of an N-terminal domain followed by two AAA ATPase domains, D1 and D2, and a C tail region (Meyer et al., 2012). The D1 and D2 domains are involved in the formation of a hexameric-double ring structure, whereas the N-terminal region of VCP is responsible for the recruitment of different cofactor proteins that enable VCP to function in several molecular pathways (Delabarre and Brunger, 2003). An autosomal dominant mutation in the D1 domain of VCP has been linked to a hereditary disease called inclusion body myopathy associated with Paget disease of bone and 
frontotemporal dementia (IBMPFD) (Watts et al., 2004). Additionally, another mutation resulting in a single amino acid substitution in VCP has been reported as a cause of familial amyotrophic lateral sclerosis (ALS) (Johnson et al., 2010). Several studies have revealed that $\mathrm{VCP}$ is a multifunctional protein that is involved in ubiquitin-mediated protein degradation, autophagy, signaling in cell cycle regulation, DNA replication, DNA repair, and ERAD system, but the functional regulation of VCP has remained unknown (Yamanaka et al., 2012). In the present study, we showed that VCP is a prominent substrate of WWP1 and WWP2. Indeed, as shown in Figure 3-16D, almost 70\% of the substrate was ubiquitinated by WWP1 in vitro. VCP is involved in dendritic spine formation as a positive regulator with neurofibromin (Lee et al., 2014). Reduced levels of VCP upon siRNA-mediated knockdown in hippocampal neurons result in impaired dendritic spine formation. Dendritic spine analysis on primary branches of apical dendrites of cortical layer II/III pyramidal neurons in NEX-Cre driven conditional double

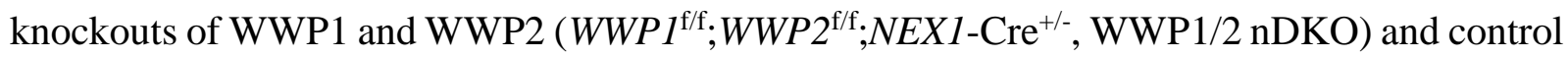
animals show that spine density in WWP1/2 nDKO is significantly increased (unpublished data from our group by Dr. Mateusz Cyryl Ambrożkiewicz). Thus, WWP1 and WWP2 might play a role in dendritic spine formation by regulating VCP function, so that deletion of WWP1 and WWP2 might result in increased VCP activity, leading to increased spine density. It would be important to study if ubiquitination deficient mutants of VCP results in high density of spines in wild type brain. This experiment would lead us to conclude the impact of ubiquitination of VCP on spine maturation. It would be also particularly important to study if reduction of VCP upon siRNA-mediated knockdown would rescue the phenotype of increased spine density in WWP1/2 nDKO mice. This experiment would lead us to conclude increased activity of VCP leads to increased spine density in WWP1/2 nDKO mice. 


\section{REFERENCES}

Ables, E.T., and Drummond-Barbosa, D. (2011). Food for thought: Neural stem cells on a diet. Cell Stem Cell 8, 352-354.

Ahmed, S.F., Deb, S., Paul, I., Chatterjee, A., Mandal, T., Chatterjee, U., and Ghosh, M.K. (2012). The chaperone-assisted E3 ligase C terminus of Hsc70-interacting protein (CHIP) targets PTEN for proteasomal degradation. J. Biol. Chem. 287, 15996-16006.

Akin, O., and Mullins, R.D. (2008). Capping Protein Increases the Rate of Actin-Based Motility by Promoting Filament Nucleation by the Arp2 / 3 Complex. 841-851.

Allen, N.J., Bennett, M.L., Foo, L.C., Wang, G.X., Chakraborty, C., Smith, S.J., and Barres, B.A. (2012). Astrocyte glypicans 4 and 6 promote formation of excitatory synapses via GluA1 AMPA receptors. Nature 486, 410-414.

Anan, T., Nagata, Y., Koga, H., Honda, Y., Yabuki, N., Miyamoto, C., Kuwano, A., Matsuda, I., Endo, F., Saya, H., et al. (1998). Human ubiquitin-protein ligase Nedd4: Expression, subcellular localization and selective interaction with ubiquitin-conjugating enzymes. Genes to Cells 3, 751-763.

Anderson, C.M., and Swanson, R.A. (2000). Astrocyte Glutamate Transport: Review of Properties, Regulation, and Physiological Functions. Glia 32, 1-14.

Arcangelo, G.D., Homayouni, R., Keshvara, L., Rice, D.S., Sheldon, M., and Curran, T. (1999). Reelin Is a Ligand for Lipoprotein Receptors. 24, 471-479.

Arevalo, J.C. (2015). Nedd4-2 regulation of voltage-gated ion channels : an update on structure - function relationships and the pathophysiological consequences of dysfunction. J Recept. Ligand Channel Res 53-63.

Arimura, N., and Kaibuchi, K. (2007a). Neuronal polarity: from extracellular signals to intracellular mechanisms. Nat. Rev. Neurosci. 8, 194-205.

Arimura, N., and Kaibuchi, K. (2007b). Neuronal polarity: from extracellular signals to intracellular mechanisms. 8 .

Bai, F., and Witzmann, F. a (2007). Synaptosome proteomics. Subcell. Biochem. 43, 77-98.

Bakkers, J., Camacho-Carvajal, M., Nowak, M., Kramer, C., Danger, B., and M., H. (2005). 
Destabilization of DeltaNp63alpha by Nedd4-mediated ubiquitination and Ubc9-mediated sumoylation, and its implications on dorsoventral patterning of the zebrafish embryo. Cell Cycle. 6, 790-800.

Barker, A.J., and Ullian, E.M. (2010). Astrocytes and synaptic plasticity. Neuroscientist 16, 4050.

Barnes, A.P., and Polleux, F. (2009). Establishment of Axon-Dendrite Polarity in Developing Neurons. Annu. Rev. Neurosci. 32, 347-381.

Barres, B.A., Koroshetz, W.J., Chun, L.L.Y., and Corey, D.P. (1990). Ion Channel Expression by White Matter Glia: The Type-1 Astrocyte. Neuron 5, 527-544.

Bartos, M., Vida, I., and Jonas, P. (2007). Synaptic mechanisms of synchronized gamma oscillations in inhibitory interneuron networks. Nat. Rev. Neurosci. 8, 45-56.

Başar, E., Başar-Eroglu, C., Karakaş, S., and Schürmann, M. (2000). Gamma, alpha, delta, and theta oscillations govern cognitive processes. Int. J. Psychophysiol. 39, 241-248.

Benediktsson, A.M., Schachtele, S.J., Green, S.H., and Dailey, M.E. (2005). Ballistic labeling and dynamic imaging of astrocytes in organotypic hippocampal slice cultures. J. Neurosci. Methods 141, 41-53.

Bezzi, P., and Volterra, A. (2001). A neuron - glia signalling network in the active brain. 387394.

Bockenhauer, D., Feather, S., Stanescu, H., Bandulik, S., Zdebik, A., Reichold, M., Tobin, J., Lieberer, E., Sterner, C., Guida, L., et al. (2009). Epilepsy, Ataxia, Sensorineural Deafness, Tubulopathy, and. N. Engl. J. Med. 360, 1960-1970.

Bragin, a, Jandó, G., Nádasdy, Z., Hetke, J., Wise, K., and Buzsáki, G. (1995). Gamma (40-100 $\mathrm{Hz}$ ) oscillation in the hippocampus of the behaving rat. J. Neurosci. 15, 47-60.

Burgering, B.M.T., and Coffer, P.J. (1995). Protein kinase B ( c-Akt ) in phosphatidylinositol3-OH Kinase signal transduction. Lett. Nat. 376, 599.

Buzsaki, G., and Wang, X. (2012). Mechanisms of Gamma Oscillations. Annu. Rev. Neurosci. $35,203-225$.

Callan, M.A., Clements, N., Ahrendt, N., and Zarnescu, D.C. (2012). Fragile X Protein is 
required for inhibition of insulin signaling and regulates glial-dependent neuroblast reactivation in the developing brain. Brain Res. 1462, 151-161.

Cao, X.R., Lill, N.L., Boase, N., Shi, P.P., Croucher, D.R., Shan, H., Qu, J., Sweezer, E.M., Place, T., Kirby, P. a, et al. (2008). Nedd4 controls animal growth by regulating IGF-1 signaling. Sci. Signal. 1, 1-10.

Carlier, A.M., Laurent, V., Santolini, J., Melki, R., Xia, G., Hong, Y., Chua, N., Pantaloni, D., Laurent, V., Santolini, J., et al. (1997). Factor ( ADF / Cofilin ) Enhances the Rate of Actin Depolymerizing Filament Turnover: Motility Implication in Actin-based. J. Cell Biol. 136, 1307-1322.

Carvalho, A.F., Pinto, M.P., Grou, C.P., Vitorino, R., Domingues, P., Yamao, F., S??-Miranda, C., and Azevedo, J.E. (2012). High-yield expression in Escherichia coli and purification of mouse ubiquitin-activating enzyme E1. Mol. Biotechnol. 51, 254-261.

Charles, A.C., Merrill, J.E., Dirksen, E.R., and Sanderson, M.J. (1991). Intercellular Signaling in Glial Cells : Calcium Waves and Oscillations in Response to Mechanical Stimulation and Glutamate. Neuron 6, 983-992.

Charles, A.C., Dirksen, E.R., Merrill, J.E., and Sanderson, M.J. (1993). Mechanisms of Intercellular Calcium Signaling in Glial Cells Studied With Dantrolene and Thapsigargin. Glia 7, 134-145.

Chell, J.M., and Brand, A.H. (2010). Nutrition-responsive glia control exit of neural stem cells from quiescence. Cell 143, 1161-1173.

Chotard, C., and Salecker, I. (2004). Neurons and glia: Team players in axon guidance. Trends Neurosci. 27, 655-661.

Christie, K.J., Martinez, J.A., and Zochodne, D.W. (2012). Disruption of E3 ligase NEDD4 in peripheral neurons interrupts axon outgrowth: Linkage to PTEN. Mol. Cell. Neurosci. 50, 179192.

Christopherson, K.S., Ullian, E.M., Stokes, C.C.A., Mullowney, C.E., Hell, J.W., Agah, A., Lawler, J., Mosher, D.F., Bornstein, P., and Barres, B.A. (2005). Thrombospondins are astrocyte-secreted proteins that promote CNS synaptogenesis. Cell 120, 421-433. 
Coco, S., Calegari, F., Pravettoni, E., Pozzi, D., Taverna, E., Rosa, P., Matteoli, M., and Verderio, C. (2003). Storage and release of ATP from astrocytes in culture. J. Biol. Chem. 278, 1354-1362.

Colgin, L.L., and Moser, E.I. (2010). Gamma oscillations in the hippocampus. Physiol. 25, 319329.

Cornell-Bell, A.N.N.H., Finkbeiner, S.M., Cooper, M.S., and Smith, S.J. (1990). Glutamate Induces Calcium Waves in Cultured Astrocytes: Longe-Range Glial Signaling. Science (80-. ). $247,470-473$.

Corty, M.M., and Freeman, M.R. (2013). Architects in neural circuit design: Glia control neuron numbers and connectivity. J. Cell Biol. 203, 395-405.

Dalton, H.E., Denton, D., Foot, N.J., Ho, K., Mills, K., Brou, C., and Kumar, S. (2011). Drosophila Ndfip is a novel regulator of Notch signaling. Cell Death Differe1. Dalt. HE, Dent. D, Foot NJ, Ho K, Mills K, Al. 2011. Drosoph. Ndfip Is a Nov. Regul. Notch Signaling. Cell Death Differ. 18(7)1150-60ntiation 18, 1150-1160.

Dammer, E.B., Na, C.H., Xu, P., Seyfried, N.T., Duong, D.M., Cheng, D., Gearing, M., Rees, H., Lah, J.J., Levey, A.I., et al. (2011). Polyubiquitin linkage profiles in three models of proteolytic stress suggest the etiology of alzheimer disease. J. Biol. Chem. 286, 10457-10465.

Danbolt, N.C. (2001). Glutamate uptake. 65, 1-105.

Dani, J.W., Chernjavsky, A., and Smith, S.J. (1992). Neuronal activity triggers calcium waves in hippocampal astrocyte networks. Neuron 8, 429-440.

Davis, D.A., Wilson, M.H., Giraud, J., Xie, Z., Tseng, H., Herscovitz, H., Tsai, L., and Delalle, I. (2009). Capzb2 Interacts with b -Tubulin to Regulate Growth Cone Morphology and Neurite Outgrowth. 7.

Dbouk, H.A., Mroue, R.M., El-sabban, M.E., and Talhouk, R.S. (2009). Cell Communication and Signaling Connexins : a myriad of functions extending beyond assembly of gap junction channels. 17.

Delabarre, B., and Brunger, A.T. (2003). Complete structure of p97 / valosin-containing protein reveals communication between nucleotide domains. 10, 856-863. 
Dere, E., Zlomuzica, A., and Binder, S. (2012). Gap Junctions in the Brain: Physiological and Pathological Roles.

Desplantez, T., Verma, V., Leybaert, L., Evans, W.H., and Weingart, R. (2012). Gap26, a connexin mimetic peptide, inhibits currents carried by connexin43 hemichannels and gap junction channels. Pharmacol. Res. 65, 546-552.

Djukic, B., Casper, K.B., Philpot, B.D., Chin, L.-S., and McCarthy, K.D. (2007). Conditional Knock-Out of Kir4.1 Leads to Glial Membrane Depolarization, Inhibition of Potassium and Glutamate Uptake, and Enhanced Short-Term Synaptic Potentiation. J. Neurosci. 27, 1135411365.

Donovan, P., and Poronnik, P. (2013). Nedd4 and Nedd4-2: Ubiquitin ligases at work in the neuron. Int. J. Biochem. Cell Biol. 45, 706-710.

Dunn, R., Klos, D.A., Adler, A.S., and Hicke, L. (2004). The C2 domain of the Rsp5 ubiquitin ligase binds membrane phosphoinositides and directs ubiquitination of endosomal cargo. J. Cell Biol. 165, 135-144.

Ekberg, J., Schuetz, F., Boase, N.A., Conroy, S., Manning, J., Kumar, S., Poronnik, P., and Adams, D.J. (2007). Regulation of the Voltage-gated K \% Channels KCNQ2 / 3 and KCNQ3 / 5 by Ubiquitination. 282, 12135-12142.

Elmariah, S.B., Oh, E.J., Hughes, E.G., and Balice-gordon, R.J. (2005). Astrocytes Regulate Inhibitory Synapse Formation via Trk- Mediated Modulation of Postsynaptic GABA A Receptors. Differences 25, 3638-3650.

Fan, Y., Tang, X., Vitriol, E., Chen, G., and Zheng, J.Q. (2011). Actin Capping Protein Is Required for Dendritic Spine Development and Synapse Formation. 31, 10228-10233.

Fellous, J.-M., and Sejnowski, T.J. (2000). Cholinergic induction of oscillations in the hippocampal slice in the slow $(0.5-2\{\mathrm{~Hz}\})$, theta $(5-12\{\mathrm{~Hz}\})$ and gamma bands. Hippocampus 10, 187-197.

Fisahn, A. (2004). Distinct Roles for the Kainate Receptor Subunits GluR5 and GluR6 in Kainate-Induced Hippocampal Gamma Oscillations. J. Neurosci. 24, 9658-9668.

Fisahn, A., Pike, F.G., Buhl, E.H., and Paulsen, O. (1998). Cholinergic induction of network 
oscillations at $40 \mathrm{~Hz}$ in the hippocampus in vitro. Nature 394, 186-189.

Fotia, A.B., Ekberg, J., Adams, D.J., Cook, D.I., Poronnik, P., and Kumar, S. (2004). Regulation of Neuronal Voltage-gated Sodium Channels by the Ubiquitin-Protein Ligases Nedd4 and Nedd4-2. J. Biol. Chem. 279, 28930-28935.

Fouladkou, F., Landry, T., Kawabe, H., Neeb, A., Lu, C., Brose, N., Stambolic, V., and Rotin, D. (2008). The ubiquitin ligase Nedd4-1 is dispensable for the regulation of PTEN stability and localization. Proc. Natl. Acad. Sci. U. S. A. 105, 8585-8590.

Frykman, S., Hur, J.-Y., Frånberg, J., Aoki, M., Winblad, B., Nahalkova, J., Behbahani, H., and Tjernberg, L.O. (2010). Synaptic and Endosomal Localization of Active $\gamma$-Secretase in Rat Brain. PLoS One 5, e8948.

Fuchs, E.C., Zivkovic, A.R., Cunningham, M.O., Middleton, S., LeBeau, F.E.N., Bannerman, D., Rozov, A., Whittington, M.A., Traub, R.D., Rawlins, J.N.P., et al. (2007). Recruitment of Parvalbumin-Positive Interneurons Determines Hippocampal Function and Associated Behavior. Neuron 53, 591-604.

Girão, H., Catarino, S., and Pereira, P. (2009). Eps15 interacts with ubiquitinated Cx43 and mediates its internalization. Exp. Cell Res. 315, 3587-3597.

Goebbels, S., Bormuth, I., Bode, U., Hermanson, O., Schwab, M.H., and Nave, K.-A. (2006). Genetic Targeting of Principal Neurons in Neocortex and Hippocampus of NEX-Cre Mice. Genesis 44, 611-621.

Goley, E.D., and Welch, M.D. (2006). The ARP2/3 complex: an actin nucleator comes of age. Nat. Rev. Mol. Cell Biol. 7, 713-726.

Gorski, J. a, Talley, T., Qiu, M., Puelles, L., Rubenstein, J.L.R., and Jones, K.R. (2002). Cortical excitatory neurons and glia, but not GABAergic neurons, are produced in the Emx1-expressing lineage. J. Neurosci. 22, 6309-6314.

Götz, M., and Huttner, W.B. (2005). The cell biology of neurogenesis. Nat Rev Mol Cell Biol $6,777-788$.

Haber, M., Zhou, L., and Murai, K.K. (2006). Cooperative Astrocyte and Dendritic Spine Dynamics at Hippocampal Excitatory Synapses. J. Neurosci. 26, 8881-8891. 
Hájos, N., and Paulsen, O. (2009). Network mechanisms of gamma oscillations in the CA3 region of the hippocampus. Neural Networks 22, 1113-1119.

Hammer, M., Krueger-burg, D., Tuffy, L.P., Rhee, J., Hammer, M., Krueger-burg, D., Tuffy, L.P., Cooper, B.H., and Taschenberger, H. (2015). Perturbed Hippocampal Synaptic Inhibition and g - Oscillations in a Neuroligin-4 Knockout Mouse Model of Autism Report Perturbed Hippocampal Synaptic Inhibition and g -Oscillations in a Neuroligin-4. CellReports 13, 516523.

Heinemann, U.W.E., and Lux, H.D. (1977). Ceiling of stimulus induced rises in extracellular potassium concentration in the cerebral cortex of cat. Brain Res. 120, 231-249.

Hertzog, M., Heijenoort, C. Van, Didry, D., Gaudier, M., Pre, T., Knossow, M., Guittet, E., Carlier, M., and Enzymologie, L. (2004). The $\square$-Thymosin / WH2 Domain : Structural Basis for the Switch from Inhibition to Promotion of Actin Assembly. 117, 611-623.

Hiesberger, T., Trommsdorff, M., Howell, B.W., Goffinet, A., Mumby, M.C., Cooper, J.A., and Herz, J. (1999). Direct Binding of Reelin to VLDL Receptor and ApoE Receptor 2 Induces Tyrosine Phosphorylation of Disabled-1 and Modulates Tau Phosphorylation. 24, 481-489.

Hrdinka, M., Draber, P., Stepanek, O., Ormsby, T., Otahal, P., Angelisova, P., Brdicka, T., Paces, J., Horejsi, V., and Drbal, K. (2011). PRR7 Is a Transmembrane Adaptor Protein Expressed in Activated T Cells Involved in Regulation of T Cell Receptor Signaling and Apoptosis * ` pa. J. Biol. Chem. 286, 19617-19629.

Hsia, H.-E., Kumar, R., Luca, R., Takeda, M., Courchet, J., Nakashima, J., Wu, S., Goebbels, S., An, W., Eickholt, B.J., et al. (2014). Ubiquitin E3 ligase Nedd4-1 acts as a downstream target of PI3K/PTEN-mTORC1 signaling to promote neurite growth. Proc. Natl. Acad. Sci. U. S. A. $111,13205-13210$.

Huang, L. (1999). Structure of an E6AP-UbcH7 Complex: Insights into Ubiquitination by the E2-E3 Enzyme Cascade. Science (80-. ). 286, 1321-1326.

Ikeda, F., and Dikic, I. (2008). Atypical ubiquitin chains: new molecular signals. "Protein Modifications: Beyond the Usual Suspects" review series. EMBO Rep. 9, 536-542.

Ingham, R.J., Gish, G., and Pawson, T. (2004). The Nedd4 family of E3 ubiquitin ligases: functional diversity within a common modular architecture. Oncogene 23, 1972-1984. 
Irobi, E., Aguda, A.H., Guerin, C., Yin, H.L., Burtnick, L.D., Blanchoin, L., and Robinson, R.C. (2004). Structural basis of actin sequestration by thymosin- b 4 : implications for WH2 proteins. 23, 3599-3608.

Jan, Y., and Jan, L.Y. (2011). Branching out : mechanisms of dendritic arborization. 11, 316328.

Jiang, H., Guo, W., Liang, X., and Rao, Y. (2005). Both the establishment and the maintenance of neuronal polarity require active mechanisms: Critical roles of GSK-3?? and its upstream regulators. Cell 120, 123-135.

Johnson, J.O., Mandrioli, J., Benatar, M., Abramzon, Y., Deerlin, V.M. Van, Trojanowski, J.Q., Gibbs, J.R., Brunetti, M., Gronka, S., Wuu, J., et al. (2010). Report Exome Sequencing Reveals VCP Mutations as a Cause of Familial ALS. 857-864.

Kam, K., and Nicoll, R. (2007). Excitatory Synaptic Transmission Persists Independently of the Glutamate - Glutamine Cycle. 27, 9192-9200.

Kang, J., Jiang, L., Goldman, S.A., and Nedergaard, M. (1998). Astrocyte-mediated potentiation of inhibitory synaptic transmission.

Katzman, R. (1976). Maintenance of a constant brain extracellular potassium. Fed Proc. 35(6), $1244-1247$.

Kawabe, H., and Brose, N. (2011). The role of ubiquitylation in nerve cell development. Nat. Rev. Neurosci. 12, 251-268.

Kawabe, H., Neeb, A., Dimova, K., Young, S.M., Takeda, M., Katsurabayashi, S., Mitkovski, M., Malakhova, O.A., Zhang, D.E., Umikawa, M., et al. (2010). Regulation of Rap2A by the Ubiquitin Ligase Nedd4-1 Controls Neurite Development. Neuron 65, 358-372.

Khvorova, A., Reynolds, A., and Jayasena, S.D. (2003). Functional siRNAs and miRNAs exhibit strand bias. Cell 115, 209-216.

Kim, H.C., and Huibregtse, J.M. (2009). Polyubiquitination by HECT E3s and the determinants of chain type specificity. Mol. Cell. Biol. 29, 3307-3318.

Kimura, K., Ito, M., Amano, M., Chihara, K., Fukata, Y., Nakafuku, M., Yamamori, B., Feng, J., Nakano, T., Okawa, K., et al. (1996). Regulation of Myosin Phosphatase by Rho and. 10-13. 
Kirischuk, S., and Ketfenmann, H. Na / Ca2 exchanger modulates signaling in Bergmann glial cells in situ. 566-572.

Kivi, A., Lehmann, T., Kovacs, R., Eilers, A., Jauch, R., Meencke, J., Deimling, A. von, Heinemann, U., and Gabriel, S. (2000). Effects of barium on stimulus-induced rises of [ K + ] o in human epileptic non-sclerotic and sclerotic hippocampal area CA1. Eur. J. Neurosci. 12, 2039-2048.

Kofuji, P., and Newman, E.A. (2004). Potassium buffering in the central nervous system. Neuroscience $129,1045-1056$.

Komander, D. (2009). The emerging complexity of protein ubiquitination. Biochem. Soc. Trans. 37, 937-953.

Kucheryavykh, Y. V, Kucheryavykh, L.Y., Nichols, C.G., Maldonado, H.M., and Baksi, K. (2007). Downregulation of Kir4 . 1 Inward Rectifying Potassium Channel Subunits by RNAi Impairs Potassium Transfer and Glutamate Uptake by Cultured Cortical Astrocytes. 281, 274281.

Kuffler, S.W. (1967). Neuroglial cells: physiological properties and a potassium mediated effect of neuronal activity on the glial membrane potential. Proc R Soc London B Biol Sci. 168, 1-21. KUFFLER, S.W., and POTTER, D.D. (1964). Glia in the Leech Central Nervous System: Physiological Properties and Neuron-Glia Relationship. J. Neurophysiol. 27, 290-320.

Kumar, S., Tomooka, Y., and Noda, M. (1992). Identification of a set of genes with developmentally down-regulated expression in the mouse brain. Biochem. Biophys. Res. Commun. 185, 1155-1161.

Kumar, S., Harvey, K.F., Kinoshita, M., Copeland, N.G., Noda, M., and Jenkins, N.A. (1997). cDNA cloning, expression analysis, and mapping of the mouse Nedd4 gene [published erratum appears in Genomics 1997 Aug 15;44(1):156]. Genomics 40, 435-443.

Lambert de Rouvroit, C., and Goffinet, A.M. (2001). Neuronal migration. Mech. Dev. 105, 4756.

LeBeau, F.E.N., Towers, S.K., Traub, R.D., Whittington, M.A., and Buhl, E.H. (2002). Fast network oscillations induced by potassium transients in the rat hippocampus in vitro. J. Physiol. 
$542,167-179$.

Lee, H.S., Ghetti, A., Pinto-Duarte, A., Wang, X., Dziewczapolski, G., Galimi, F., HuitronResendiz, S., Pina-Crespo, J.C., Roberts, A.J., Verma, I.M., et al. (2014). Astrocytes contribute to gamma oscillations and recognition memory. Proc. Natl. Acad. Sci. 111, E3343-E3352.

Lee, K.A., Hammerle, L.P., Andrews, P.S., Stokes, M.P., Mustelin, T., Silva, J.C., Black, R.A., and Doedens, J.R. (2011). Ubiquitin Ligase Substrate Identification through Quantitative Proteomics at Both the Protein and Peptide. 286, 41530-41538.

Lemus, L., and Goder, V. (2014). Regulation of Endoplasmic Reticulum-Associated Protein Degradation (ERAD) by Ubiquitin. Cells. 3, 824-847.

Leykauf, K., Salek, M., Bomke, J., Frech, M., Lehmann, W.-D., Dürst, M., and Alonso, A. (2006). Ubiquitin protein ligase Nedd4 binds to connexin43 by a phosphorylation-modulated process. J. Cell Sci. 119, 3634-3642.

Liang, S., Carlson, G.C., and Coulter, D.A. (2006). Dynamic Regulation of Synaptic GABA Release by the Glutamate-Glutamine Cycle in Hippocampal Area CA1. 26, 8537-8548.

Lin, X., Ruan, X., Anderson, M.G., McDowell, J.A., Kroeger, P.E., Fesik, S.W., and Shen, Y. (2005). siRNA-mediated off-target gene silencing triggered by a $7 \mathrm{nt}$ complementation. Nucleic Acids Res. 33, 4527-4535.

Litterman, N., Ikeuchi, Y., Gallardo, G., Connell, B.C.O., Sowa, M.E., Gygi, S.P., Harper, J.W., and Bonni, A. (2011). An OBSL1-Cul7 Fbxw8 Ubiquitin Ligase Signaling Mechanism Regulates Golgi Morphology and Dendrite Patterning. 9, 17-19.

Lu, C.B., Hamilton, J.B., Powell, A.D., Toescu, E.C., and Vreugdenhil, M. (2011). Effect of ageing on CA3 interneuron sAHP and gamma oscillations is activity-dependent. Neurobiol. Aging 32, 956-965.

Macvicar, B.A., and Tse, F.W.Y. (1988). Norepinephrine and Cyclic Adenosine 3 '': 5 ' -Cyclic Monophosphate Enhance a Nifedipine-Sensitive Calcium Current in Cultured Rat Astrocytes. Glia 1, 359-365.

Maddika, S., Kavela, S., Rani, N., Palicharla, V.R., Pokorny, J.L., Sarkaria, J.N., and Chen, J. (2011). WWP2 is an E3 ubiquitin ligase for PTEN. Nat. Cell Biol. 13, 728-733. 
Mann, E.O., and Mody, I. (2009). Control of hippocampal gamma oscillation frequency by tonic inhibition and excitation of interneurons. Nat. Neurosci. 13, 205-212.

Mann, E.O., Suckling, J.M., Hajos, N., Greenfield, S.A., and Paulsen, O. (2005). Perisomatic feedback inhibition underlies cholinergically induced fast network oscillations in the rat hippocampus in vitro. Neuron 45, 105-117.

Marín-Teva, J.L., Dusart, I., Colin, C., Gervais, A., Van Rooijen, N., and Mallat, M. (2004). Microglia Promote the Death of Developing Purkinje Cells. Neuron 41, 535-547.

Marrero, H., Astion, M.L., Coles, J.A., and Orkand, R.K. (1989). Facilitation of voltage-gated ion channels in frog neuroglia by nerve impulses. Nature 339, 378-380.

Maspero, E., Valentini, E., Mari, S., Cecatiello, V., Soffientini, P., Pasqualato, S., and Polo, S. (2013). Structure of a ubiquitin-loaded HECT ligase reveals the molecular basis for catalytic priming. Nat. Struct. Mol. Biol. 20, 696-701.

Mathalon, D.H., and Sohal, V.S. (2015). Neural Oscillations and Synchrony in Brain Dysfunction and Neuropsychiatric Disorders It's About Time. JAMA Psychiatry 4.

Matsui, K., and Jahr, C.E. (2006). Exocytosis unbound. 305-311.

Mauch, D.H., Nägler, K., Schumacher, S., Göritz, C., Müller, E.C., Otto, a, and Pfrieger, F.W. (2001). CNS synaptogenesis promoted by glia-derived cholesterol. Science 294, 1354-1357.

Meyer, H., Bug, M., and Bremer, S. (2012). Emerging functions of the VCP / p97 AAA-ATPase in the ubiquitin system. Nat. Publ. Gr. 14, 117-123.

Mothet, J.-P., Pollegioni, L., Ouanounou, G., Martineau, M., Fossier, P., and Baux, G. (2005). Glutamate receptor activation triggers a calcium-dependent and SNARE protein-dependent release of the gliotransmitter D-serine. Proc. Natl. Acad. Sci. U. S. A. 102, 5606-5611.

Mulder, J., Ariaens, A., Boomen, D. Van Den, and Moolenaar, W.H. (2004). p116 Rip Targets Myosin Phosphatase to the Actin Cytoskeleton and Is Essential for RhoA / ROCK-regulated Neuritogenesis. 15, 5516-5527.

Murata, Y., Doi, T., Taniguchi, H., and Fujiyoshi, Y. (2005). Proteomic analysis revealed a novel synaptic proline-rich membrane protein (PRR7) associated with PSD-95 and NMDA receptor. 327, 183-191. 
Na, C.H., Jones, D.R., Yang, Y., Wang, X., Xu, Y., and Peng, J. (2012). Synaptic Protein Ubiquitination in Rat Brain Revealed by Antibody- based Ubiquitome Analysis.

Neusch, C., Rozengurt, N., Jacobs, R.E., Lester, H. a, and Kofuji, P. (2001). Kir4.1 potassium channel subunit is crucial for oligodendrocyte development and in vivo myelination. J. Neurosci. 21, 5429-5438.

Neusch, C., Papadopoulos, N., Müller, M., Maletzki, I., Winter, S.M., Hirrlinger, J., Handschuh, M., Bähr, M., Richter, D.W., Kirchhoff, F., et al. (2006). Lack of the Kir4.1 channel subunit abolishes $\mathrm{K}+$ buffering properties of astrocytes in the ventral respiratory group: impact on extracellular K+ regulation. J. Neurophysiol. 95, 1843-1852.

Newman, E. a (2003). Glial cell inhibition of neurons by release of ATP. J. Neurosci. 23, 16591666.

Nichols, C.G., and Lopatin, A.N. (1997). Inward Rectifier Potassium Channels. Annu Rev Physiol. 59, 171-191.

Nicholson, C., and Syková, E. (1998). Extracellular space structure revealed by diffusion analysis. TINS 21, 207-214.

Nicholson-Dykstra, S., Higgs, H.N., and Harris, E.S. (2005). Actin dynamics: Growth from dendritic branches. Curr. Biol. 15, 346-357.

O’donnell, M., Chance, R.K., and Bashaw, G.J. (2009). Axon growth and guidance: receptor regulation and signal transduction. Annu. Rev. Neurosci. 32, 383-412.

Ogawa, M., and Miyata, T. (1995). The reeler Gene-Associated Antigen on CajaI-Retzius Neurons Is a Crucial Molecule for Laminar Organization of Cortical Neurons. 14, 899-912.

Ohno, Y., Hibino, H., Lossin, C., Inanobe, A., and Kurachi, Y. (2007). Inhibition of astroglial Kir4.1 channels by selective serotonin reuptake inhibitors. Brain Res. 1178, 44-51.

Olsen, M.L., and Sontheimer, H. (2008). Functional implications for Kir4.1 channels in glial biology: From K + buffering to cell differentiation. J. Neurochem. 107, 589-601.

Olsen, M.L., Higashimori, H., Campbell, S.L., Hablitz, J.J., and Sontheimer, H. (2006). Functional Expression of K ir 4 . 1 Channels in Spinal Cord Astrocytes. 528, 516-528.

Orkand, R.K., Nicholls, J.G., and Kuffler, S.W. (1966). EFFECT OF NERVE IMPULSES ON 
THE MEMBRANE POTENTIAL OF GLIAL CELLS IN THE CENTRAL NERVOUS SYSTEM. J. Neurophysiol. 29, 788-806.

Panatier, A., Theodosis, D.T., Mothet, J.P., Touquet, B., Pollegioni, L., Poulain, D.A., and Oliet, S.H.R. (2006). Glia-Derived d-Serine Controls NMDA Receptor Activity and Synaptic Memory. Cell 125, 775-784.

Park, H., Yang, J., Kim, R., Li, Y., Lee, Y., Lee, C., Park, J., Lee, D., Kim, H., and Kim, E. (2015). Mice lacking the PSD-95 - interacting E3 ligase , Dorfin / Rnf19a , display reduced adult neurogenesis, enhanced long- term potentiation, and impaired contextual fear conditioning. Nat. Publ. Gr. 1-16.

Parpura, V., Basarsky, T.A., Liu, F., Ksenija, J., Jeftinija, S., and Haydon, P.G. (1994). Glutamate-mediated astrocyt-neuron signaling. Nature 369, 744-747.

Parri, H.R., Gould, T.M., and Crunelli, V. (2001). Spontaneous astrocytic Ca $2+$ oscillations in situ drive NMDAR- mediated neuronal excitation.

Persaud, A., Alberts, P., Amsen, E.M., Xiong, X., Wasmuth, J., Saadon, Z., Fladd, C., Parkinson, J., and Rotin, D. (2009). Comparison of substrate specificity of the ubiquitin ligases Nedd4 and Nedd4-2 using proteome arrays. Mol. Syst. Biol. 5, 333.

Persaud, A., Alberts, P., Hayes, M., Guettler, S., Clarke, I., Sicheri, F., Dirks, P., Ciruna, B., and Rotin, D. (2011). Nedd4-1 binds and ubiquitylates activated FGFR1 to control its endocytosis and function. EMBO J. 30, 3259-3273.

Plant, P.J., Yeger, H., Staub, O., Howard, P., and Rotin, D. (1997). The C2 domain of the ubiquitin protein ligase Nedd4 mediates Ca2+- dependent plasma membrane localization. J. Biol. Chem. 272, 32329-32336.

Pollard, T.D., Borisy, G.G., and Haven, N. (2003). Cellular Motility Driven by Assembly and Disassembly of Actin Filaments. 112, 453-465.

Puram, S. V, and Bonni, A. (2013). Cell-intrinsic drivers of dendrite morphogenesis. 46574671.

Rakic, P. (1971). Neuron-glia relationship during granule cell migration in developing cerebellar cortex. A Golgi and electronmicroscopic study in Macacus Rhesus. J. Comp. Neurol. 141, 283- 
Ransom, B.S., and Goldring, S. (1973). Slow hyperpolarization in cells to be glia in cerebral cortex of cat. J Neurophysiol 36(5), 879-892.

Robel, S., and Sontheimer, H. (2015). Glia as drivers of abnormal neuronal activity. Nat. Neurosci. 19, 28-33.

Rotin, D., and Kumar, S. (2009). Physiological functions of the HECT family of ubiquitin ligases. Nat. Rev. Mol. Cell Biol. 10, 398-409.

Rouach, N., and Giaume, C. (2001). Connexins and gap junctional communication in astrocytes are targets for neuroglial interaction. 132.

Rougier, J.-S., Bemmelen, M.X. Van, Bruce, M.C., Jespersen, T., Gavillet, B., Apothe, F., Cordonier, S., Staub, O., Rotin, D., Abriel, H., et al. (2005). Molecular determinants of voltagegated sodium channel regulation by the Nedd4 / Nedd4-like proteins. Am J Physiol Cell Physiol 208, 692-701.

Rougier, J.-S., Albesa, M., Abriel, H., and Viard, P. (2011). Neuronal Precursor Cell-expressed Developmentally Down-regulated 4-1 (NEDD4-1) Controls the Sorting of Newly Synthesized CaV1.2 Calcium Channels. J. Biol. Chem. 286, 8829-8838.

Sakata, T., Sakaguchi, H., Tsuda, L., Higashitani, A., Aigaki, T., Matsuno, K., and Hayashi, S. (2004). Drosophila Nedd4 Regulates Endocytosis of Notch and Suppresses Its LigandIndependent Activation. Curr. Biol. 14, 2228-2236.

Salm, A.K., and McCarthy, K.D. (1990). Norepinephrine-Evoked Calcium Transients in Cultured Cerebral Type 1 Astroglia. Glia 3, 529-538.

Sasaki, S., Shionoya, A., Ishida, M., Gambello, M.J., Yingling, J., Wynshaw-boris, A., and Hirotsune, S. (2000). A LIS1 / NUDEL / Cytoplasmic Dynein Heavy Chain Complex in the Developing and Adult Nervous System. 28, 681-696.

Schafer, D. a, and Cooper, J. a (1995). Control of actin assembly at filament ends. Annu. Rev. Cell Dev. Biol. 11, 497-518.

Scheffner, M., and Kumar, S. (2014). Mammalian HECT ubiquitin-protein ligases: Biological and pathophysiological aspects. Biochim. Biophys. Acta - Mol. Cell Res. 1843, 61-74. 
Schmidt, C., Hesse, D., Raabe, M., Urlaub, H., and Jahn, O. (2013). An automated in-gel digestion/iTRAQ-labeling workflow for robust quantification of gel-separated proteins. Proteomics 13, 1417-1422.

Scholl, U.I., Choi, M., Liu, T., Ramaekers, V.T., Häusler, M.G., Grimmer, J., Tobe, S.W., Farhi, A., Nelson-Williams, C., and Lifton, R.P. (2009). Seizures, sensorineural deafness, ataxia, mental retardation, and electrolyte imbalance (SeSAME syndrome) caused by mutations in KCNJ10. Proc. Natl. Acad. Sci. U. S. A. 106, 5842-5847.

Schuetz, F., Kumar, S., Poronnik, P., and Adams, D.J. (2008). Regulation of the voltage-gated K 2 channels KCNQ2 / 3 and KCNQ3 / 5 by serum- and glucocorticoid-regulated kinase-1. 7380.

Schwamborn, J.C., and Püschel, A.W. (2004). The sequential activity of the GTPases Rap1B and Cdc42 determines neuronal polarity. Nat. Neurosci. 7, 923-929.

Scott, E.K., and Luo, L. (2001). How do dendrites take their shape ? 15, 359-365.

Scudder, S.L., Goo, M.S., Cartier, A.E., Molteni, A., Schwarz, L.A., Wright, R., and Patrick, G.N. (2014). Synaptic strength is bidirectionally controlled by opposing activity-dependent regulation of Nedd4-1 and USP8. J. Neurosci. 34, 16637-16649.

Seifert, G., and Steinhäuser, C. (2013). Neuron - astrocyte signaling and epilepsy. Exp. Neurol. 244, 4-10.

Sekine, K., Kawauchi, T., Kubo, K., Honda, T., Herz, J., and Hattori, M. (2012). Article Reelin Controls Neuronal Positioning by Promoting Cell-Matrix Adhesion via Inside-Out Activation of Integrin a 5 b 1 . Neuron 76, 353-369.

Senzaki, K., Ogawa, M., and Yagi, T. (1999). Proteins of the CNR Family Are Multiple Receptors for Reelin. 99, 635-647.

Shapiro, L.A., Korn, M.J., Shan, Z., and Ribak, C.E. (2005). GFAP-expressing radial glia-like cell bodies are involved in a one-to-one relationship with doublecortin-immunolabeled newborn neurons in the adult dentate gyrus. Brain Res. 1040, 81-91.

Shi, S.H., Jan, L.Y., and Jan, Y.-N. (2003). Hippocampal neuronal polarity specified by spatially localized mPar3/mPar6 and PI 3-kinase activity. Cell 112, 63-75. 
Shi, S.-H., Cheng, T., Jan, L.Y., and Han, Y.-N. (2004). APC and GSK-3? Are Involved in mPar3 Targeting to the Nascent Axon and Establishment of Neuronal Polarity. Curr. Biol. 14, 2025-2032.

Shigetomi, E., Tong, X., Kwan, K.Y., Corey, D.P., and Khakh, B.S. (2011). TRPA1 channels regulate astrocyte resting calcium and inhibitory synapse efficacy through GAT-3. Nat. Neurosci. $15,70-80$.

Shigetomi, E., Jackson-Weaver, O., Huckstepp, R.T., O’Dell, T.J., and Khakh, B.S. (2013). TRPA1 Channels Are Regulators of Astrocyte Basal Calcium Levels and Long-Term Potentiation via Constitutive D-Serine Release. J. Neurosci. 33, 10143-10153.

Sholl, D.A. (1953). Dendritic organization in the neurons of the visual and motor cortices of the cat. J Anat 387-406.

da Silva, J.S., and Dotti, C.G. (2002). Breaking the neuronal sphere: regulation of the actin cytoskeleton in neuritogenesis. Nat Rev Neurosci 3, 694-704.

Snijders, T.M., Milivojevic, B., and Kemner, C. (2013). NeuroImage: Clinical Atypical excitation - inhibition balance in autism captured by the gamma response to contextual modulation. YNICL 3, 65-72.

Somjen, G.G. (1979). Extracellular potassium in the mammalian central nervous system. Annu Rev Physiol. 41, 159-177.

Soroceanu, L., Manning, T.J., and Sontheimer, H. (2001). Reduced expression of connexin-43 and functional gap junction coupling in human gliomas. Glia 33, 107-117.

Stout, C.E., Costantin, J.L., Naus, C.C.G., and Charles, A.C. (2002). Intercellular Calcium Signaling in Astrocytes via ATP Release through Connexin Hemichannels. J. Biol. Chem. 277, 10482-10488.

Suadicani, S.O., Flores, C.E., Urban-Maldonado, M., Beelitz, M., and Scemes, E. (2004). Gap junction channels coordinate the propagation of intercellular $\mathrm{Ca} 2+$ signals generated by P2Y receptor activation. Glia 48, 217-229.

Svitkina, T.M., and Borisy, G.G. (1999). Arp2/3 complex and actin depolymerizing factor/cofilin in dendritic organization and treadmilling of actin filament array in lamellipodia. 
J. Cell Biol. 145, 1009-1026.

Tang, E.I., Mok, K., Lee, W.M., and Cheng, C.Y. (2016). Networks at the Sertoli Cell BloodTestis Barrier in Male Rats : An In Vitro Study. 156, 680-693.

Theis, M., Soehl, G., Speidel, D., Kuehn, R., and Willecke, K. (2003a). Connexin43 is not expressed in principal cells of mouse cortex and hippocampus. 18, 267-274.

Theis, M., Jauch, R., Zhuo, L., Speidel, D., Wallraff, A., Do, B., Frisch, C., So, G., Teubner, B., Euwens, C., et al. (2003b). Accelerated Hippocampal Spreading Depression and Enhanced Locomotory Activity in Mice with Astrocyte-Directed Inactivation of Connexin43. 23, 766776.

Van Themsche, C., Leblanc, V., Parent, S., and Asselin, E. (2009). X-linked inhibitor of apoptosis protein (XIAP) regulates PTEN ubiquitination, content, and compartmentalization. J. Biol. Chem. 284, 20462-20466.

Tian, M., Bai, C., Lin, Q., Lin, H., Liu, M., Ding, F., and Wang, H.R. (2011). Binding of RhoA by the $\mathrm{C} 2$ domain of E3 ligase Smurf1 is essential for Smurf1-regulated RhoA ubiquitination and cell protrusive activity. FEBS Lett. 585, 2199-2204.

Trommsdorff, M., Gotthardt, M., Hiesberger, T., Shelton, J., Stockinger, W., Nimpf, J., Hammer, R.E., Richardson, J.A., and Herz, J. (1999). Reeler / Disabled-like Disruption of Neuronal Migration in Knockout Mice Lacking the VLDL Receptor and ApoE Receptor 2. 97, 689-701.

Trotman, L.C., Wang, X., Alimonti, A., Chen, Z., Teruya-Feldstein, J., Yang, H., Pavletich, N.P., Carver, B.S., Cordon-Cardo, C., Erdjument-Bromage, H., et al. (2007). Ubiquitination Regulates PTEN Nuclear Import and Tumor Suppression. Cell 128, 141-156.

Usowicz, M.M., Gallo, V., and Cull-Candy, S.G. (1989). Multiple conductance channels in type-2 cerebellar astrocytes activated by excitatory amino acids. Nature 339, 380-383.

Venance, L., Piomelli, D., Glowinski, J., and Giaume, C. (1995). Inhibition by anandamide of gap junctions and intracellular calcium signaling in striatal astrocytes. Lett. to Nat. 376, 590594.

Venance, L., Stella, N., Glowinski, J., and Giaume, C. (1997). Mechanism Involved in Initiation 
and Propagation of Receptor- Induced Intercellular Calcium Signaling in Cultured Rat Astrocytes. 17, 1981-1992.

Verkhratsky, A., and Butt, A.M. (2013). Neuroglia: Definition, Classification, Evolution, Numbers, Development 3.1. Glial Physiol. an Pathophysiol. 73-104.

Verkhratsky, A., and Kirchhoff, F. (2007). NMDA Receptors in Glia. 13.

Vignjevic, D., Yarar, D., Welch, M.D., Peloquin, J., Svitkina, T., and Borisy, G.G. (2003). Formation of filopodia-like bundles in vitro from a dendritic network. J. Cell Biol. 160, 951962.

Virchow, R. (1856). Uber das granulirte Ansehen der Wandungen der Gehirnventrikel. Gesammelte Abhandlungen Zur Wissenshaftlichen Medizin, Frankfurt 885-891.

Vreugdenhil, M., and Toescu, E.C. (2005). Age-dependent reduction of?? oscillations in the mouse hippocampus in vitro. Neuroscience 132, 1151-1160.

Wallraff, A. (2006). The Impact of Astrocytic Gap Junctional Coupling on Potassium Buffering in the Hippocampus. J. Neurosci. 26, 5438-5447.

Wang, X., Trotman, L.C., Koppie, T., Alimonti, A., Chen, Z., Gao, Z., Wang, J., ErdjumentBromage, H., Tempst, P., Cordon-Cardo, C., et al. (2007). NEDD4-1 Is a Proto-Oncogenic Ubiquitin Ligase for PTEN. Cell 128, 129-139.

Watts, G.D.J., Wymer, J., Kovach, M.J., Mehta, S.G., Mumm, S., Darvish, D., Pestronk, A., Whyte, M.P., and Kimonis, V.E. (2004). Inclusion body myopathy associated with Paget disease of bone and frontotemporal dementia is caused by mutant valosin-containing protein. 36, 377381.

Wear, M.A., Yamashita, A., Kim, K., Mae, Y., Cooper, J.A., and Louis, S. (2003). How Capping Protein Binds the Barbed End of the Actin Filament. 13, 1531-1537.

Wetherington, J., Serrano, G., and Dingledine, R. (2008). Astrocytes in the Epileptic Brain. Neuron 58, 168-178.

Whittington, M. a, Traub, R.D., and Jefferys, J.G. (1995). Synchronized oscillations in interneuron networks driven by metabotropic glutamate receptor activation. Nature 373, 612615. 
Wiesner, S., Ogunjimi, A.A., Wang, H.R., Rotin, D., Sicheri, F., Wrana, J.L., and Forman-Kay, J.D. (2007). Autoinhibition of the HECT-Type Ubiquitin Ligase Smurf2 through Its C2 Domain. Cell 130, 651-662.

Winder, S.J., Kathryn, R., Winder, S.J., and Ayscough, K.R. (2005). Actin-binding proteins Actin-bindin g Protein s. 2005, 651-654.

Winter, C.G., Wang, B., Ballew, A., Royou, A., Karess, R., Axelrod, J.D., Luo, L., and Yvette, G. (2001). Drosophila Rho-Associated Kinase ( Drok ) Links Frizzled-Mediated Planar Cell Polarity Signaling to the Actin Cytoskeleton. 105, 81-91.

Xu, P., Duong, D.M., Seyfried, N.T., Cheng, D., Xie, Y., Robert, J., Rush, J., Hochstrasser, M., Finley, D., and Peng, J. (2009). Quantitative Proteomics Reveals the Function of Unconventional Ubiquitin Chains in Proteasomal Degradation. Cell 137, 133-145.

Yamada, M., Ohnishi, H., Sano, S., Nakatani, A., Ikeuchi, T., and Hatanaka, H. (1997). Insulin Receptor Substrate (IRS)-1 and IRS-2 Are Tyrosine-phosphorylated and Associated with Phosphatidylinositol 3-Kinase in Response to Brain-derived Neurotrophic Factor in Cultured Cerebral Cortical Neurons. J. Biol. Chem. 272, 30334-30339.

Yamamoto, T., Vukelic, J., Hertzberg, E., and Nagy, J.I. (1992). Differential anatomical and cellular patterns of connexin43 expression during postnatal development of rat brain. Brain Res. $66,165-180$.

Yamanaka, K., Sasagawa, Y., and Ogura, T. (2012). Recent advances in p97 / VCP / Cdc48 cellular functions ì . Biochim. Biophys. Acta - Mol. Cell Res. 1823, 130-137.

Yan, D., Guo, L., and Wang, Y. (2006). Requirement of dendritic Akt degradation by the ubiquitin-proteasome system for neuronal polarity. J. Cell Biol. 174, 415-424.

Yu, T., Calvo, L., Southon, E., Saray, L., Chao, M. V, and Tessarollo, L. (2011). Regulation of Trafficking of Activated TrkA Is Critical for NGF-Mediated Functions. 2, 521-534.

Yu, T., Calvo, L., Lo, S., Lo, R., Vicente-garcía, C., Tessarollo, L., Rodriguez, R.E., and Are, J.C. (2014). In Vivo Regulation of NGF-Mediated Functions by Nedd4-2 Ubiquitination of TrkA. 34, 6098-6106. 


\section{ACKNOWLEDGEMENT}

I would like to express my gratitude to people who support me through my doctoral study. First, I thank Prof. Nils Brose for embracing me as a member of Department of Molecular Neurobiology, for his critical scientific input and for constant encouragement. It was a pleasure to develop as a scientist in his lab. Nils, thank you for your support to make this work possible and your contribution to its success.

I would like to express my sincere gratitude to my supervisor, Dr. Hiroshi Kawabe, for including me as a member of his group and introducing me the projects described in this thesis. Hiroshi, thank you for your encouragement, guidance, training in experimental skills and long scientific discussions even in the late evenings or over the weekends. Above all, thank you for urging me to success better after I have already tried my best and for making me devoted to science.

I would like to thank Prof. Nils Brose and Dr. Hiroshi Kawabe again for their insightful comments and remarks on this thesis.

I thank my thesis committee members, Dr. Judith Stegmüller and Prof. Dr. Dirk Görlich for their critical evaluation and valuable input on my projects during the regular meetings.

Also, I would like to express my gratitude towards IMPRS Neuroscience coordination office, our godmother and godfather, Sandra Drube and Prof. Dr. Micheal Hörner for their immense support and for creating an inspiring atmosphere in the IMPRS Neuroscience program. Sandra, you have made my life much easier in the most stressful and desperate situations, thank you for all your effort and extra work that I caused you to do. Micheal, I will be always thankful to you for your constant guidance through all my time at IMPRS Neuroscience Program.

I sincerely thank Jennifer Day and Lydia Bickford for proof reading this manuscript. Lydia, thank you for also bringing me a new computer from US. Indeed, I wrote the whole my thesis with my new computer.

It would like to thank Hung-En Hsia, Mateusz Ambrozkiewicz, Manuela Schwark, Oykum Kaplan, Michiko Takeda and Bernd Hesse-Neißen who are former and present members of the Kawabe Group. I would also like to express my gratitude to members of the 
Ubiquitin/SUMO subgroup for having fruitful scientific discussion. I am also deeply thankful to Klaus-Peter Hellmann for his excellent technical assistance.

I would like to acknowledge the support of all members of Department of Molecular Neurobiology and thank them for creating a scientific atmosphere that I enjoyed my time during my doctorate. Special thanks to Mateusz, Silvia, Hung-En, Mimi, Anja, Ben, Matthieu, Dilja, James, Olga, Noa, Theo, Marylin, Liam, Cordelia, Caro. I would like to specially thank HongJun and Manu for chocolate times and enjoyable discussions at the third floor balcony.

I would like to express my gratitude towards all members of AGCT lab, Fritz Benseller, Ivonne Thanhäuser, Dayana Schwerdtfeger, Christiane Harenberg and Maik Schlip, who made this work much efficient and faster by their constant patience and help for genotyping, DNA sequencing and oligonucleotide synthesis. I am also grateful to the staff of the MPI-EM Animal House Facility for the maintenance of the mouse lines.

I would like to express my special gratitude to our collaborators. First, and foremost, I would sincerely thank Anes Ju and Dr. Jeong Seop Rhee for their laborious and elegant input for electrophysiological experiments. I would like to thank Dr. Jeong Seop Rhee for his valuable scientific input and his advice for my scientific studies. I would like to thank Dr. Irina Majoul for providing Connexin-43 antibody. I would like to thank Dr. Yoshinori Fujiyoshi for Prr7 antibody and constructs. I would like to thank Dr. Johannes Hirrlinger for Kir4.1 antibody and constructs.

Furthermore, I would like to thank my German bro, David, and my German mom, Gabi. You made it more possible to live in abroad. David, I will always remember our study times and 'challenged accepted' moments during first year of our studies in Neuroscience Program. Many thanks also my dearest friends, Diana and Chaitali for their constant support during all of this time. Also, I would like to thank to lovely members of Monsterparty group, Anes, Fernanda, Giulia, Barbara, Edda, Livia, Mara, Marie and Ludo for their support and for making life in Göttingen more fun.

I would like to express my exceptional gratitude to Dr. Tolga Soykan for his unprecedented professionalism, for his mental support throughout this projects. Tolga, you became my older brother that I did not have it before. You always listened all my problems and 
supported me with an extraordinary patience. I cannot thank you enough. I will always remember our 'in vino veritas' times.

I would like to thank Burak K., Utku K., Ahmet B. K., members of Quatro Irmaos for everything. You guys became my family in Göttingen and always supported me in very exhausting moments. Siz bana kardeş olmak için aile olmak için kan bağına gerek olmadığını gösterdiniz. Her daim yanımda oldunuz. Burak baba, umutsuz kaldığım zamanlarda cesaretlendirici mesajlarını, abi tavsiyelerini hiç unutmayacağım, her daim abimiz kal. Utku K., sen her zaman o güzel ve nadide şakalarınla en kötü anlarda bile yüzümüze gülücükler kondurmayı bırakma. Ahmet sende bize insanın ne kadar rahat ve mutlu olabileceğini her daim göster kardeşim. İyi ki varsınız.

Canım Bekir hocam, Özgün öğretmenim, İrem ve Ekin, Avşar ailesi olarak hep yanımda oldunuz ve beni her daim desteklediniz. Bazen sesinizi duymak, hatta varlığınız bilmek bile yetti güçlü olmam için. Herşey için çok ama çok teşekkür ederim.

Canım annem, biricik babam, sevgili kardeşlerim Emine ve Mahmut, sizden uzaklarda, çok zorlu dönemlerde bana hep destek oldunuz. Anne, baba sizler benim her şeyimsiniz. Beni dünyaya getiren, büyüten ve bu günlere gelmemi sağlayan insanlarsınız. Beni ben yapan sizlersiniz. Sizlere ne kadar teşekkür etsem az. Emine ve Mahmut, sizler benim her daim güvenebileceğim, herşeyi paylaşabileceğim canım kardeşlerimsiniz. Sizlere çok teşekkür ederim. 


\section{LIST OF ABBREVIATIONS}

\begin{tabular}{|l|l|}
\hline ADF & Actin-depolymerizing factor \\
\hline AKT1 & RAC-alpha serine/threonine-protein kinase \\
\hline AMP & Adenosine-5'-monophosphate \\
\hline AMPAR & a-amino-3-hydroxy-5-methyl-4isoxazolepropionic acid receptor \\
\hline APOER2 & Apolipoprotein E receptor 2 \\
\hline ARP & Actin-related protein \\
\hline ATP & Adenosine-5'-triphosphate \\
\hline BP & Basal progenitor \\
\hline CAPZA & F-actin capping protein alpha \\
\hline CNS & Central nervous system \\
\hline CRK & Mitogen-activated protein kinase 14 \\
\hline CRKL & Crk-like protein \\
\hline C-terminal & Carboxy-terminal \\
\hline CUX & Homeobox protein cut-like \\
\hline DAB1 & Disabled Homologue 1 \\
\hline DIV & Day in vitro \\
\hline DNA & Deoxyribonucleic acid \\
\hline DUB & Deubiquitinase \\
\hline E. coli & Escherichia coli \\
\hline E1 & Ubiquitin activating enzyme \\
\hline E2 & Ubiquitin conjugating enzyme \\
\hline E3 & Ubiquitin ligase \\
\hline EGFP & Enhanced green fluorescent protein \\
\hline EMX1 & Empty spiracles homolog 1 \\
\hline EPSC & Excitatory postsynaptic current \\
\hline ERAD & Endoplasmic reticulum-associated degradation \\
\hline F-actin & Fibrilar actin \\
\hline FYN & Tyrosine-protein kinase Fyn \\
\hline GABA & Gamma-aminobutyric acid \\
\hline G-actin & Globular actin \\
\hline GLAST & Sodium-dependent glutamate/aspartate transporter 1 \\
\hline GLT1 & Glutamate Transporter 1 \\
\hline GPCR & G-protein coupled receptors \\
\hline GSK3 $\beta$ & Serine/threonine-protein kinase-3 beta \\
\hline GST & Glutathione S-transferase \\
\hline HA & Human influenza hemagglutinin \\
\hline HECT & Homologous to E6-AP C terminus \\
\hline HRP & Horseradish peroxidase \\
\hline
\end{tabular}




\begin{tabular}{|c|c|}
\hline ICW & Intercellular Calcium Waves \\
\hline IP & Immunoprecipitation \\
\hline IPSC & Inhibitory postsynaptic current \\
\hline KAR & Kainic acid receptor \\
\hline $\mathrm{KD}$ & Knock-down \\
\hline $\mathrm{kDa}$ & Kilo Dalton \\
\hline Kir & Inwardly Rectifying Potassium Channel \\
\hline $\mathrm{KO}$ & Knock-out \\
\hline LIS1 & Lissencephaly type 1 \\
\hline mGluR & Metabotrophic Glutamate Receptor \\
\hline mRNA & Messenger Ribonucleic acid \\
\hline mTORC1 & $\begin{array}{l}\text { Serine/threonine protein kinase mammalian target of rapamycin } \\
\text { complex } 1\end{array}$ \\
\hline $\mathrm{MZ}$ & Marginal zone \\
\hline Nedd4 & Neuronal precursor cell expresse developmentally down-regulated 4 \\
\hline Nedd4-1 bKO & Nedd4-1 ${ }^{\mathrm{ff}} ;$ EMX1-Cre \\
\hline Nedd4-1/2 bDKO & Nedd4-1 $1^{\mathrm{f} / \mathrm{f}} ;$ Nedd4-2 ${ }^{\mathrm{f} / \mathrm{f}} ;$ EMX1-Cre \\
\hline Nedd4-1/2 nDKO & Nedd4-1 $1^{\mathrm{f} / \mathrm{f}} ; \mathrm{Nedd} 4-2^{\mathrm{f} / \mathrm{f}} ; \mathrm{NEX} 1-\mathrm{Cre}$ \\
\hline Nedd4-2 bKO & Nedd4-2 ${ }^{\mathrm{f} / \mathrm{f}} ; \mathrm{EMX1-Cre}$ \\
\hline NEX1 & Neurogenix differentiation factor 6 \\
\hline $\mathrm{NgCAM}$ & Neuron-glia cell adhesion molecule \\
\hline NGF & Nerve Growth Factor \\
\hline NMDA & N-Methyl-D-aspartic acid \\
\hline NMDAR & N-Methyl-D-aspartic acid receptor \\
\hline NR & N-Methyl-D-aspartic acid receptor subunit \\
\hline NT & Neurotrophin \\
\hline N-terminal & Amino-terminal \\
\hline N-WASP & Neural Wiskott-Aldrich syndrome protein \\
\hline PI3K & Phophoinositide 3-kinase \\
\hline PKM2 & Pyruvate kinase M2 \\
\hline PLC & Phospholipase C \\
\hline $\operatorname{Prr} 7$ & Proline rich protein 7 \\
\hline PSD & Postsynaptic density \\
\hline PtdInsP2 & Phosphatidylinositol 4,5-biphosphate \\
\hline PtdInsP3 & Phophotidylinositol-3,4,5-triphospahte \\
\hline PTEN & Phosphatase and tensin homolog \\
\hline Rab2A & Ras-related protein $2 \mathrm{~A}$ \\
\hline RGC & Radial Glial Cell \\
\hline RING & Really interesting new gene \\
\hline
\end{tabular}




\begin{tabular}{|l|l|} 
RNA & Ribonucleic acid \\
\hline ROK & Rho-associated kinase \\
\hline RT & Room temperature \\
\hline SDS-PAGE & Sodium dodecyl sulfate polyacrylamide gel electrophoresis \\
\hline SEM & Standard error of mean \\
\hline Smurf & SMAD ubiquitination regulatory factor 1 \\
\hline SRC & Neuronal proto-oncogene tyrosine-protein kinase Src \\
\hline SVET1 & Netrin receptor UNC5D \\
\hline SVZ & Subventricular zone \\
\hline TBR2 & T-box brain protein 2 \\
\hline Ub & Ubiquitin \\
\hline VCP & Valosine Containing Protein \\
\hline VLDLR & Very low-density lipoprotein receptor \\
\hline VZ & Ventricular zone \\
\hline WASP & Wiskott-Aldrich syndrome protein homolog \\
\hline WAVE & Wiskott-Aldrich syndrome protein family member \\
\hline WB & Western Blotting \\
\hline WT & Wild type \\
\hline WWP & WW containing protein \\
\hline
\end{tabular}




\section{CURRICULUM VITAE}

Personal information

Date of birth $\quad 13.02 .1988$

Place of birth Kirsehir, Turkey

Nationality Turkish

Address Max Planck Institute for Experimental Medicine

Hermann-Rein-Str. 3

37075, Göttingen

Germany

Phone number $\quad$ (+49) 5513899714

Email_altas@em.mpg.de

\section{Education}

April 2012- Current

Ph.D. student at the M.Sc./Ph.D. IMPRS Neuroscience program Göttingen, Germany. Project title: "Roles of Nedd4 Family E3 Ligases in Glial Function and Nerve Cell Development". Supervisor Dr. Hiroshi Kawabe

\section{September 2010- March 2012}

M.Sc. student at the M.Sc./Ph.D. IMPRS Neuroscience program Göttingen, Germany. M.Sc. thesis

September 2005- June 2010

B.Sc. in Molecular Biology and Genetics at the Middle East Technical University, Ankara, Turkey.

\section{Research Projects}

Lab rotation; 'Glutamate Uptake by VLUTs into Reconstituted Proteoliposomes', supervised by Dr. Julia Preobraschenski and Prof. Dr. Reinhard Jahn, Max Planck Institute of Biophysical Chemistry, Department of Neurobiology 
Lab rotation; 'Impact of BDNF on synaptic vesicle recycling in excitatory and inhibitory presynaptic regions', supervised by Dr. Camin Dean, European Neuroscience Institute, Göttingen, Department of Transsynaptic Signaling

2011

Lab rotation; 'Silencing of Otoferlin Expression by microRNAs', supervised by Dr. Iliana Panou and Prof. Dr. Tobias Moser, Inner Ear Laboratory, Department of Otolaryngology

\section{Conferences}

May 2013

May 2013

Sep 2012
Neurizons 2013: Solving the Brain Puzzle- organizer.

Annual Meeting of the SPP1365-2 - presentation

ZOMES VII Ubiquitin-like proteins and their cognate PCI complexes

\section{Teaching experience}

Feb 2016

Feb 2015

Feb 2015

June-Sep 2014
Tutor for the lectures on 'Signal Transduction I and II' for Neuroscience

Tutor for the lectures on 'DNA/Genome' and

'Transcription/RNA/Translation' for IMPRS Neuroscience

Tutor for the lectures on 'Signal Transduction I and II' for IMPRS Neuroscience

Supervisor of internship student Mehmet Can Demirler

\section{Extra-Cullicular 2013}

2013
Co-organizer of Neurizons 2013 conference, Max Planck Institute for Biophysical Chemisrty Göttingen

$\mathrm{PhD}$ Student Representative, Max Planck Institute for Experımental Medicine 


\section{$9 \quad$ LIST OF PUBLICATIONS}

Altas, B., Jahn, O., and Kawabe, H., Biochemical Purification of Binding Partners of Synaptic Scaffold Proteins. Methods in Molecular Biology. Manuscript in revision.

Ott, C., Martens, H., Hassouna, I., Oliveira, B., Erck, C., Zafeiriou, M.-P., Peteri, U.-K., Hesse, D., Gerhart, S., Altas, B., et al. (2015). Widespread Expression of Erythropoietin Receptor in Brain and Its Induction by Injury. Mol. Med. 21, 803-815.

Shinoda, Y., Ahmed, S., Ramachandran, B., Bharat, V., Brockelt, D., Altas, B., and Dean, C. (2014). BDNF enhances spontaneous and activity-dependent neurotransmitter release at excitatory terminals but not at inhibitory terminals in hippocampal neurons. Front. Synaptic Neurosci. 6, 27. 